\title{
Symphony on Strong Field Approximation
}

Kasra Amini, ${ }^{1,2}$ Jens Biegert, ${ }^{2,3}$ Francesca Calegari, ${ }^{4,5}$ Alexis Chacón, ${ }^{6}$ Marcelo F. Ciappina, ${ }^{7}$ Alexandre Dauphin, ${ }^{2}$ Dmitry K. Efimov, ${ }^{8}$ Carla Figueira de Morisson Faria,${ }^{9}$ Krzysztof Giergiel, ${ }^{8}$ Piotr Gniewek, ${ }^{1}$ Alexandra S. Landsman, ${ }^{10,11}$ Michał Lesiuk, ${ }^{1}$ Michał Mandrysz, ${ }^{8}$ Andrew S. Maxwell,${ }^{9}$ Robert Moszyński, ${ }^{1}$ Lisa Ortmann, ${ }^{12}$ Jose Antonio Pérez-Hernández, ${ }^{13}$ Antonio Picón, ${ }^{2,}{ }^{14}$ Emilio Pisanty, ${ }^{2}$ Jakub Prauzner-Bechcicki, ${ }^{8}$ Krzysztof Sacha, ${ }^{8,}{ }^{15}$ Noslen Suárez, ${ }^{2}$ Amelle Zaïr, ${ }^{16}$ Jakub Zakrzewski, ${ }^{8,}{ }^{15}$ and Maciej Lewenstein ${ }^{2,3, *}$

${ }^{1}$ Faculty of Chemistry, University of Warsaw, Pasteura 1, 02-093 Warsaw, Poland

${ }^{2}$ ICFO - Institut de Ciencies Fotoniques, The Barcelona Institute

of Science and Technology, 08860 Castelldefels (Barcelona), Spain

${ }^{3}$ ICREA, Pg. Lluís Companys 23, 08010 Barcelona, Spain

${ }^{4}$ Center for Free-Electron Laser Science, DESY, Notkestr. 85, 22607 Hamburg, Germany

${ }^{5}$ CNR, Instituto di Fotonica e Nanotecnologie Milano, Piazza L. da Vinci 32, 20133 Milano, Italy

${ }^{6}$ Theoretical Division, Los Alamos National Laboratory, Los Alamos, New Mexico 87545, USA

${ }^{7}$ Institute of Physics of the ASCR, ELI-Beamlines project, Na Slovance 2, 182 21 Prague, Czech Republic

${ }^{8}$ Instytut Fizyki imienia Mariana Smoluchowskiego,

Uniwersytet Jagielloński, Eojasiewicza 11, 30-348 Kraków, Poland

${ }^{9}$ Department of Physics \& Astronomy, University College London,

Gower Street London WC1E 6BT, United Kingdom

${ }^{10}$ Max-Planck Institut für Physik Komplexer Systeme, Nöthnitzer-Strasse 38, D-01187 Dresden, Germany

${ }^{11}$ Department of Physics, Max Planck Postech, Pohang, Gyeongbuk 37673, Republic of Korea

${ }^{12}$ Max-Planck Institut für Physik komplexer Systeme, Nöthnitzer-Strasse 38, D-01187 Dresden, Germany

${ }^{13}$ Centro de Láseres Pulsados (CLPU), Parque Científico, E-37185 Villamayor, Salamanca, Spain

${ }^{14}$ Departamento de Química, Universidad Autónoma de Madrid, 28049, Madrid, Spain

${ }^{15}$ Mark Kac Complex Systems Research Center, Jagiellonian University, Eojasiewicza 11, 30-348 Kraków, Poland

${ }^{16}$ Faculty of Natural 83 Mathematical Sciences Department of Physics,

King's College London, Strand Campus, London WC2R 2LS, UK

(Dated: January 1, 2019)

This paper has been prepared by the Symphony collaboration (University of Warsaw, Uniwersytet Jagielloński, DESY/CNR and ICFO) on the occasion of the 25th anniversary of the "simple man's models" which underlie most of the phenomena that occur when intense ultrashort laser pulses interact with matter. The phenomena in question include High-Harmonic Generation, Above-Threshold Ionization, and Non-Sequential Multielectron Ionization. "Simple man's models" provide, both an intuitive basis for understanding the numerical solutions of the time-dependent Schrödinger equation, and the motivation for the powerful analytic approximations generally known as the Strong Field Approximation (SFA). In this paper we first review the SFA in the form developed by us in the last 25 years. In this approach SFA is a method to solve the TDSE using a systematic perturbation theory in a part of the Hamiltonian describing continuum-continuum transitions in the presence of the laser field. In this review we focus on recent applications of SFA to HHG, ATI and NSMI from multi-electron atoms and from multi-atom. The main novel part of the presented theory concerns generalizations of SFA to: (i) time-dependent treatment of two-electron atoms, allowing for studies of an interplay between Electron Impact Ionization (EII) and Resonant Excitation with Subsequent Ionization (RESI); (ii) time-dependent treatment in the single active electron (SAE) approximation of âĂIJlargeâĂ molecules and targets which are themselves undergoing dynamics during the HHG or ATI process. In particular, we formulate the general expressions for the case of arbitrary molecules, combining input from quantum chemistry and quantum dynamics. We formulate also theory of time-dependent separable molecular potentials to model analytically the dynamics of realistic electronic wave packets for molecules in strong laser fields.

We dedicate this work to the memory of Bertrand Carré, who passed away in March 2018 at the age of 60 .

*maciej.lewenstein@icfo.eu 


\section{INTRODUCTION}

\section{A. Motivation}

In the last three decades, we have witnessed incredible advances in laser technology and in the understanding of nonlinear laser-matter interactions, crowned recently by the award of the Nobel prize to Gérard Mourou and Donna Strickland $[1,2]$. It is now routinely possible to produce few-cycle femtosecond $\left(1 \mathrm{fs}=10^{-15} \mathrm{~s}\right)$ laser pulses in the visible and mid-infrared regimes [3, 4]. By focusing such ultrashort laser pulses on gas or solid targets, possibly in a presence of nano-structures [5], the targets are subjected to an ultra-intense electric field, with peak field strengths approaching the binding field inside the atoms themselves. Such fields permit the exploration of the interaction between strong electromagnetic coherent radiation and an atomic or molecular system with unprecedented spatial and temporal resolution [6]. On one hand, HHG nowadays can be used to generate attosecond pulses in the extreme ultraviolet [7, 8], or even in the soft X-ray regime [9]. Such pulses themselves may be used for dynamical spectroscopy of matter; despite carrying modest pulse energies, they exhibit excellent coherence properties [10, 11]. Combined with femtosecond pulses they can also be used to extract information about the laser pulse electric field itself [12]. HHG sources therefore offer an important alternative to other sources of XUV and X-ray radiation: synchrotrons, free electron lasers, X-ray lasers, and laser plasma sources. Moreover, HHG pulses can provide information about the structure of the target atom, molecule or solid [13-15]. Of course, to decode such information from a highly nonlinear HHG signal is a challenge, and that is why a possibly perfect, and possibly "as analytical as possible" theoretical understanding of these processes is in high demand. Here is the first instance where SFA offers its basic services.

Since electronic motion is governed by the waveform of the laser electric field, an important quantity to describe the electric field shape is the so-called absolute phase or carrier-envelope phase (CEP). Control over the CEP is paramount for extracting information about electron dynamics, and to retrieve structural information from atoms and molecules $[13,16,17]$. For instance, in HHG an electron is liberated from an atom or molecule through ionization, which occurs close to the maximum of the electric field. Within the oscillating field, the electron can thus accelerate along oscillating trajectories, which may result in recollision with the parent ion, roughly when the laser field approaches a zero value. Control over the CEP is particularly important for HHG, when targets are driven by laser pulses comprising only one or two optical cycles. In that situation the CEP determines the relevant electron trajectories, i.e. the CEP determines whether emission results in a single or in multiple attosecond bursts of radiation [16, 18].

The influence of the CEP on electron emission is also extremely important. It was demonstrated for instance in an anti-correlation experiment, in which the number of ATI electrons emitted in opposite directions was measured [12, 19]. Since the first proof-of-principle experiment [12], the stereo ATI technique has established itself as a direct measure of the CEP, and demonstrated its ability for single-shot measurements even at multi-kHz laser repetition rates. Both bound-free and the rescattering continuum-continuum transitions are CEP sensitive; hence, the photoelectron distribution of ATI can also be used to extract structural information about the target. Again, "as analytical as possible" theoretical understanding of these processes and SFA are more than welcome here.

Laser induced electron diffraction (LIED) is the technique that uses the doubly-differential elastic scattering crosssections to extract structural information [20-22]. Meeting the requirements to extract structural information has, however, proven difficult due to the stringent prerequisites on the laser parameters. During recent years, the development of new laser sources has dramatically advanced, leading to the first demonstrations of the technique [15, 23-26], and the successful retrieval of the bond distances in simple diatomic molecules with fixed-angle broadband electron scattering [23]. Recently, Pullen et al. [15] have exploited the full double differential cross section to image the entire structure of a polyatomic molecule for the first time. Again, to exploit the full potential of the recollision physics and the intrinsic time resolution of LIED (and eventually HHG), we need the comprehensive and complete understanding of the ATI and HHG processes and its theoretical, possibly analytical description [19, 27-32]. Here is the third instance where SFA is indispensable.

The key for gaining dynamical structural information and for realizing nonlinear dynamical spectroscopy with HHG, ATI and, last but not least, NSMI, however, consists in generalizations of the existing theories to the case, when the target in question undergoes itself dynamics beyond its SAE electronic structure. For NSMI this requires including two, or even more electrons in the SFA theory. For molecules that would mean, for instance, developing theory that takes into account vibration or dissociation processes, occurring on the time scale comparable with the laser pulse duration. Ab initio theory of this kind is generally too computationally intensive for numerical simulation. Generalizations of SFA to include many electrons and/or nuclear motion are thus more than welcome. 


\section{B. History of SFA}

The history of SFA has at least five beginnings, many intertwined and entangled paths, and many interfering endings. The point of view presented here is dominated by the opinion of one of us (M.L.), who started to work on the subject in 1980. The five beginnings of SFA, according to this point of view, are the following:

- Keldysh ionization theory. In 1964 L.V. Keldysh [33] attempted and solved analytically a very fundamental problem of tunnelling ionization of atoms in strong low frequency oscillating electric fields. In the epochal paper entitled "Ionization in the Field of a Strong Electromagnetic Wave" he derived his famous Bessel function formulae for ionizations rates. F.H.M. Faisal [27] and H.R. Reiss [28] extended this theory to calculate electron spectra in the process to be named Above-Threshold ionization (ATI). These results were the first instances of the Strong Field Approximation, named "KFR theory" after the authors. Note that in this initial phase, SFA was exclusively applied to ionization problems. The first experiments on ATI date 1979 [34]; in fact, the name of the observed phenomenon slowly shifted from "continuum-continuum transitions" to ATI [35-37]. The first observations of HHG date 1988 [38, 39], and of non-sequential two-electron ionization originate from 1983 [40, 41; for more recent reviews see 42, 43]. Keldysh theory was further developed to calculate total ionization rates for various atomic species and states by M.V. Ammosov, N.B. Delone and V.P. Krainov [44] - the resulting expressions are known as ADK rates. More broadly, Keldysh theory has been an inspiration for many years for many scholars. M.L. first learned the Keldysh theory from a preprint from ICTP Trieste by L. Davidovich et al.. In fact, Davidovich later published several interesting papers on Keldysh theory [45-47]. In the beginning of the 1980s several non-perturbative, quantum-optics-inspired models of "continuum-continuum transitions" and ATI were introduced by Z. Białynicka-Birula [48], J.H. Eberly[49], K. Rzążewski [50] and others (for a review see Ref. 51). These models stimulated M.L. to try to combine them with the Keldysh theory.

- Kroll-Watson theory. Another inspiration for the contemporary SFA came from the seminal papers of N.M. Kroll and K.M. Watson on electron-atom [52] and atom-atom [53] scattering in the presence of a strong electromagnetic wave. The earlier paper clearly dealt with the problem of "continuum-continuum transitions", dressed by the laser field, leading to the expected Bessel-function dependence of the corresponding transition amplitudes. This observation led to the formulations of the ATI theory as a theory of multichannel decay and continuum-continuum transitions, dressed by the laser field [54]. This approach, employing the relation between ATI and electron scattering in the intense laser field has been deepened and developed further in Ref. 55. In the contemporary language, the results of these studies described ATI as a combination of direct tunnelling processes, and rescattering processes occurring in the laser dressed continuum. At that time, however, the underlying quasi-classical theory and the simple man's model was yet not known. It is worth noting that this approach was also applied to two-electron ionization in Ref. 56, where the direct two-electron tunnelling processes were analysed.

- Numerical studies of TDSE. Numerical simulations always played, play and will play a fundamental role in understanding of physics of matter in intense laser fields. A particularly important role was played here by 1D models of one- and two-electron systems, initiated by J.H. Eberly on "Eberlonium", also known as the Rochester atom model. This series of studies allowed the description of several qualitatively important results but, more importantly, it allowed - by appropriate tuning of the parameters - the finding of accurate quantitative predictions concerning ATI [57], HHG [58, 59], stabilization of an 1D atom in strong field of high frequency [60] - all of that optimizing the "model atom for multiphoton physics" [61]. This approach was very successfully generalized to $1 \mathrm{D}$ two-electron models [62, 63], which in turn stimulated the development of other quasi-1D approaches to the two-electron problems in intense laser fields. These developments were very important, especially in a view of the difficulties and computational cost of solving TDSE for helium in 3D [64-67; see also 68, 69]. Analysis of the classical pathways for simultaneous escape of two electrons showed that there are two saddle points located symmetrically with respect to the field polarization axis [70]. This led to a modification of the 1D model, where electrons move along axes inclined symmetrically with respect to the polarization direction [71-73]. Within this model the ionization for three active electrons was also recently considered [74]. Another model, in which the center of mass movement was restricted to the polarization axis was introduced by Ruiz et al. [75], and successfully applied to momentum distributions [76] (for a comparison of various quasi-1D approaches see Ref. 77).

Nevertheless, the most important were investigations of the TDSE in 3D, led in those years by K. Kulander, who not only developed codes for solving TDSE, but also propagation and phase matching in HHG, and collaborated intensively with the top experimental groups. Several seminal papers were written on ATI [78], double ionization of helium [79, 80] and phase matching in HHG [81]. The one that was the most important for the formulation of the simple man's models was the theory paper on HHG from atoms and ions in the high-intensity regime [82], 
in which the famous cut-off law for HHG was discovered: the high harmonics cease to exist above the photon energy $I_{p}+3.2 U_{p}$, where $I_{p}$ is the ionization potential, and $U_{p}$ is the ponderomotive energy.

- Classical phase-space methods. The key idea in these approaches was to mimic the evolution of the electronic system in terms of classical trajectories, governed by the completely classical Hamiltonians, but satisfying an initial phase-space distribution. In fact, that is why some versions of this approach were termed the "truncated Wigner function" approach. Initially these methods were proposed for microwave perturbations of atomic systems by J. Leopold and I.C. Percival [83, 84]. They gained a lot of attention and popularity in the studies of quantum chaos and quantum dynamical localization [85]. Later, these methods were extended to the regime of mid-infrared, and even high laser frequencies and two-electron systems. There are two variants of these methods that can be distinguished: those where the initial distribution is calculated classically [86-90], and those which use the below-barrier tunneling approximations for the calculation of the initial distribution [91]. The quality of classical phase-space averaging methods can be checked by comparing their results with those from the corresponding quantum-mechanical models [92]. A considerable progress was made with these methods for study of the ionization yields [93, 94] and momentum distributions [95]. Still, getting HHG spectra within classical methods exclusively seems to be a complicated task (for a recent discussion see Ref. 96).

- Simple man's models. There were simple man's models before the simple man's model. An extended discussion of precursors is contained in a recent review at Ref. 97, where the earlier quantum formulation of "Atomic Antennas" of M.Y. Kuchiev is discussed [98], as well as early attempts by F. Brunel and P. Corkum himself [99-101]. Essentially the same formulae as the ones derived later in the framework of SFA for HHG were obtained by F. Ehlotzky [102], but without the underlying quasi-classical picture. The history of science chooses its own heroes. Nowadays, the simple man's model, also known as the "three-step" or "recollision" model, is usually attributed to P. Corkum [103], K. Kulander [82, 104], and to a conference contribution of H. Muller. These formulations were done in the right place in the right time, and were truly seminal - they have revolutionized the whole area! M.L. learned about the simple man's model for HHG during and immediately after the famous NATO Workshop on Super Intense Laser Atom Physics (SILAP) in Han-sur-Lesse in Belgium in January of 1993 [105]. After the workshop M. Yu. Ivanov visited Saclay and stayed at M.L.'s house - that is how our first paper on the SFA for HHG, based on simple man's model, and co-authored by A. L'Huillier and other colleagues, was written [106]. After a long fight with Phys. Rev. Lett., this paper was published as a Rapid Communication in Phys. Rev. A, entitled "High-oder Harmonic-generation cutoff". We termed the formula we used to evaluate the time-dependent dipole moment a dynamical Landau-Dykhne formula.

M.L. went to JILA in February 1993 and started to work on the long version of the theory, including a detailed discussion of the relation of the simple man's model with the quasi-classical (better termed quantum-orbit) saddle point approximations, along with concrete calculations for what we called Gaussian models, i.e. models in which the ground state of the atom of interest was approximated by a Gaussian function. The paper on the theory of HHG by the low frequency laser fields appeared in Phys. Rev. A in 1994, and soon became a reference paper for theorists and experimentalists working in the field [107]. During his stay in JILA M.L. worked with K. Kulander on the extension of the newly-formulated version of the SFA to ATI, stimulated by the observation of the intensity-dependent rings in the high order ATI [108]. The paper that extended a quasi-classical analysis (i.e. one based on simple man's model) of rescattering processes in ATI appeared in Phys. Rev. A in 1995 [29]. On one hand, it explicitly demonstrated in which sense the SFA is a systematic perturbation theory in part of the Hamiltonian describing the continuum-continuum transitions. On the other hand, we introduced here for the first time the model atom involving a separable (non-local) potential. This kind of approach was a generalization of the so-called zero-range Becker's model [109]. Recently, it turned out to be extremely useful in modelling HHG and ATI in molecular dimers, trimers and quadrimers [110-114]. Other uses of separable potentials in the literature are discussed in Refs. 115-129.

The quantum simple man's models, as the novel SFA was termed sometimes, proved very useful in explaining the relation of quantum orbits to phase matching in HHG. It allowed thus to realise coherence control in high-order harmonics [130], and understand the behaviour of the phase of the atomic polarization in high-order harmonic generation [131], which in turn allowed the construction of schemes for generation of attosecond pulse trains using HHG [132]. The first such trains were observed in by P. Agostini et consortes in 2001 [133]. Equally well, the quantum simple man's model allowed for explanations, both intuitive and quantitatively accurate, of the generation of a single isolated attosecond pulse by an ultrashort, few-cycle laser pulse [7, 134-136].

The crowning of the SFA applications in the 1990s was perhaps the Science paper [137], in which theory was confronted with the experimental results of the groups of the late B. Carré and P. Salières at Saclay, on quantum orbits in HHG, and of G. Paulus and H. Walther at MPI Garching, on quantum orbits in ATI, driven by elliptically-polarized laser fields. 


\section{SFA today}

Since the formulation of various versions of SFA, starting from Keldysh in 1964-1965 until the approaches based on simple man's models, formulated in 1993-1994, SFA kept being one of the most important theoretical tools of the physics of matter in intense laser fields. There are several important review articles and books that either describe or include description of developments and applications of SFA [6, 19, 138, 139]. There were various directions in which the theory of SFA developed in the recent 20 years, with many authors working on technical issues of improving the accuracy of the theory, while others generalised the theory to novel regions. We summarize here some of the main trends in this body of work:

- Coulomb corrections. A number of strong-field phenomena, particularly in ionization experiments, show features caused by the ion's Coulomb potential that evade the SFA, ranging from Coulomb focusing [140] and the asymmetric photoelectron spectra produced by elliptical polarizations [141] to the more recent "ionization surprise" of the so-called Low-Energy Structures [142]. Early work focused on including the Coulomb potential through a Born series, often with a single rescattering used very successfully for the ATI plateau and NSDI [143], but this is generally insufficient for Coulomb-dependent phenomena. Current approaches include the use of an oscillating Coulomb-wave basis for the continuum (the Coulomb-Volkov approximation [144]), the eikonal inclusion of the Coulomb Hamiltonian to solve the continuum TDSE (the analytical $R$-matrix theory [145]) and the direct modification of the SFA's trajectory language to include Coulomb potential influence on the action and the trajectories (the Coulomb-Corrected SFA [146]). In addition to more "phenomenological" approaches (cf. Refs. 147, 148) there have been very elegant approaches based on the Feynman path integral formulation [149]. The most recent results based on this approach are discussed in Refs. 150-153.

- Saddle point techniques. The simple man's model's classical trajectories are encoded in the SFA as the quantum orbits obtained as the saddle-point contributions to the oscillatory integrals. Understanding the nature of these saddle points in the complex time and momentum planes [154-156] allows for a clear understanding of the coherent contribution of each pathway [137], and it also paves the way for experiments showing the contributions of other orbits [157, 158]. Technical improvements include the regularization of discontinuities at the cutoff via uniform approximations [159, 160], and the extension to multi-electron configurations [97]. On the other hand, some problems, such as the inclusion of field dressing of the ground state [161], are less amenable to saddle-point analysis.

- Applications to novel systems: Two-electron systems. In the last two decades SFA has been successfully applied to two-electron systems. Most of these approaches used SFA formulations based on $S$-matrix theory à la W. Becker [138, 154], while the use of the physics of strong laser fields for imaging goes back to the seminal references 13 (for $\mathrm{HHG}$ ) and 162 (for ATI). In fact, one could argue that two-electron experiments on cold target recoil ion momentum spectroscopy (COLTRIMS) [163] pioneered the imaging methods using strong-field physics. The phenomenon of interest here is the Non-Sequential Double ionization (NSDI), which occurs in accordance with P. Corkum's idea of a recollision-driven model [103]. Still, NSDI has two faces. If the ionization potential of the target ion is smaller than $3.17 U_{p}$, the recolliding electron may directly cause stripping of another electron, since there is enough energy for that; this scenario is called Electron Impact ionization (EII). On the other hand, if the recolliding electron does not have enough energy, it may still excite the target ion to an excited state, from which direct tunneling might easily take place; this scenario is known as Recollision Excitation with Subsequent ionization (RESI). In EII electrons are typically ejected step by step, most of the quantum interference effects are washed out, and the standard SFA and quasi-classical trajectory models work very well [76, 138, 164-167]. The early studies of the RESI observed that time delay leads to back-to-back electron ejection, and it was assumed that interferences between different channels (different intermediate excited states, etc.) were irrelevant [168, 169]. Pretty soon, however, a myriad of shapes electron momentum distributions were observed in RESI and, moreover, experiments were in clear contrast to the simplest SFA theories [170174]. It was then realised that the interference must be accounted for in RESI [175-177]. A lot of insight was gained by the sophisticated analysis of the saddle-point approach [178]. A more complete understanding of the RESI phenomenon, taking into account interference effects, was only achieved recently [151, 172, 179]. All these results allow, in principle, to work backwards toward the experimental data to reconstruct the states of the excited electron involved in RESI. Amazingly, the channel interference in RESI seems to have been observed recently [180].

- Applications to novel systems: Atto-nanophysics. In the last decade SFA has been successfully applied to situations, in which HHG, ATI or NSMI come directly from nano-structures, via for instance plasmonic excitations, or from atomic/molecular targets located close to nano-structures. In the latter case, plasmonic 
enhanced electromagnetic fields close to the structures serve to excite the targets. Recent development of this area, termed atto-nanophysics are extensively described in the review [5].

- Applications to novel systems: Solid state. In the past 5 years, the strong field community has increasingly turned its attention to condensed matter systems, following the observation of high-order harmonics from bulk crystals subjected to strong laser fields [181]. Our understanding of dynamics in gases, based essentially on SFA, has recently been extended to yield crucial insights into microscopic attosecond phenomena in condensed matter [182-184]. The merger of strong field physics with solids has the potential to revolutionize contemporary electronics [185], as well as yield crucial fundamental insights into long-standing problems in condensed matter physics. For instance, the first direct measurements of the Berry phase [186, 187] were accomplished using HHG. These recent measurements promise to be of great interest to the condensed-matter community, due to the important role played by Berry phase in the anomalous Hall effect and in topological insulators, among other fields.

- Applications to novel systems: Large molecules. In a series of recent papers we revisited the SFA model for ATI for few-cycle infrared (IR) laser pulses [110-114]. We compared it first with the numerical solution of the TDSE in one (1D) and two (2D) spatial dimensions for an atomic system [29]. We developed and generalized there an analytical atomic model based on a non-local (short range) potential. In the first paper [110] we analysed ATI for an atom, followed by ATI [111] and HHG [112] for diatomic molecules. Here we paid special attention to non-physical terms which arise in the theory if plane waves are used instead of the exact continuum scattering states of the system. Finally, in Refs. 113 and 114, we generalized our approach to molecular trimers and quadrimers, and attempted to describe Laser Induced Electron Diffraction for such targets. The ultimate goal of this theory is to characterize the time evolution of the target (its size, configuration, its molecular orbital, its dynamical configuration, etc.) by looking at the ATI spectrum and angular distributions, especially in the region of high energies, corresponding to rescattering processes.

- Applications to novel systems: quantum simulators. The strong-field dynamics described by the SFA have very close analogues in the motion of cold atoms in optical traps [188], particularly via the KramersHenneberger correspondence between a dipole coupling and a 'shaken' atomic potential [189]. In the decades since, cold trapped atoms have become one of the primary platforms for quantum simulation [190], and several works have explored the possibility of using cold-atom simulators to probe the strong-field dynamics described by SFA [191-194], thereby allowing a complementary look at observables (like e.g. the instantaneous wavefunction, or a full quantum state tomography on the outgoing particles) that are inaccessible to conventional experiments. While some dedicated experimental efforts to implement this are still at the tool-building stage [195], there are already functioning quantum simulation platforms for ultrafast physics [196], which should provide growing opportunities to test SFA physics in new ways. Similar complementary views on strong-field dynamics should also be available via photonic simulation, using the natural Schrödinger-equation correspondence for optical fibers [197].

\section{The present paper}

The present article is organized as follows. Section I, Introduction, covers the motivations for the paper (Subsection I A), and the past (Subsection IB) and present (Subsection IC) of SFA. In Section II we present a short explainer of the basic phenomena and processes: HHG, ATI and NSMI, including representative and explanatory figures. In Section III we review in more detail the theory, which describes the HHG and ATI processes within the version of Strong Field Approximation following Ref. 107. In particular, we present the derivation of the transition amplitudes for both the direct and the rescattered electrons, as well as for the time-dependent dipole moment. We develop in detail the mathematical foundations towards the final results by starting from the Hamiltonian, which describes the atomic system and the TDSE associated to it. Section IV is devoted entirely to two-electron processes. We derive for the first time SFA for this case in an explicit time-dependent approach, and we analyze the interplay between the EII and RESI processes.

In Section V, we formulate our theory for the case when the single active electron (SAE) approximation is applied to a molecule undergoing temporal evolution of its nuclear configuration. This is done using the Born-Oppenheimer approximation and classical equations of motion for the nuclei. We consider first the simplest case when the molecule's dynamics (vibrations, dissociations) do not affect the SAE electronic dynamics. Even in this simple case novel effects appear in the SFA dynamics, such as the appearance of a temporal Berry phase. In Subsection VB we go beyond this approximation and consider the self-consistent dynamics, in which SAE dynamics affect nuclei and vice versa. We conclude in Subsection VC where we present an outlook on extending our quasi-analytical model to more complex 
atomic and molecular systems. Finally, in Section VI we briefly review the recent application of SFA theory to the generation of harmonics in solid-state systems.

Appendix A discusses time dependent ADK rates. In Appendix B we sketch calculations of $a(t)$ - the amplitude of the ground state within our generalized SFA theory. In Appendix C we introduce the model for our atomic and molecular systems that uses a particular form of a non-local short-range separable potential. The matrix elements to describe the direct ionization and the re-scattering processes are then computed analytically.

We then offer additional material regarding the two-electron theory: in Appendix D we we discuss the properties of the dipole matrix elements involved, and in Appendix E we pose full forms for the two-electron integro-differential equations derived from the TDSE. additionally we include Appendix F, where we present solutions of the RESI and EII equations using additional approximations for the dipole matrix elements, neglecting electron-electron interaction effects for those elements.

\section{STRONG-FIELD PROCESSES IN AN ATOMIC GAS}

Over the lifetime of strong-field physics, SFA theory has accounted for a broad variety of physical phenomena which could not be explained by traditional perturbation theory. These phenomena involve light-matter interaction using laser fields of field strengths that are comparable to the Coulomb force of attraction between electrons and protons. Consequently, this can lead to the distortion of the Coulomb potential and the subsequent lowering of the barrier to ionization in the strong-field regime. Many non-linear ionization processes can be initiated in this regime, such as multi-photon ionization (MPI), above-threshold ionization (ATI), tunnel ionization (TI), and over-the-barrier (OTB) ionization. These processes are shown in Fig. 1 with their operating conditions summarized in Table I. The ionization regime of operation can be identified by the Keldysh parameter, $\gamma$, given by

$$
\gamma=\sqrt{\frac{I_{p}}{2 U_{p}}},
$$

where $I_{p}$ is the ionization potential (i.e. the energy required to eject an electron from the ground state to the ionization continuum), and $U_{p}$ is the ponderomotive energy (i.e. the average kinetic energy of the oscillations of a free electron in a laser field) given by

$$
U_{\mathrm{p}}=\frac{e^{2} \mathcal{E}_{0}^{2}}{4 m_{e} \omega_{0}^{2}}=\frac{I_{0} e^{2} \lambda_{0}^{2}}{8 \pi^{2} m_{\mathrm{e}} \epsilon_{0} c^{3}}=9.337 \times 10^{-20} I_{0} \lambda_{0}^{2} \frac{\mathrm{eV}}{\mathrm{W} \mathrm{cm}^{-2} \mathrm{~nm}^{2}}
$$

where $e$ is the elementary charge, $\mathcal{E}_{0}$ is the electric field amplitude, $m_{\mathrm{e}}$ is the mass of an electron, $\omega_{0}, \lambda_{0}$ and $I_{0}$ are the central laser frequency, wavelength and intensity, respectively, $\epsilon_{0}$ is the vacuum permittivity, and $c$ is the speed of light. We give, in the rest of this section, a brief summary of strong-field processes that are based on ATI and TI.

(a) Field Free

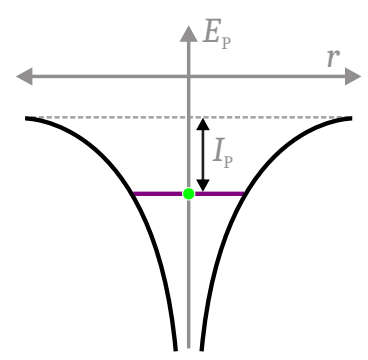

(b) Multi-photon Ionization

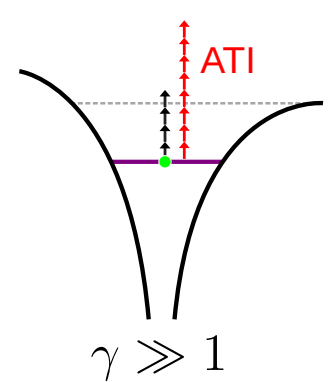

(c) Tunnel Ionization

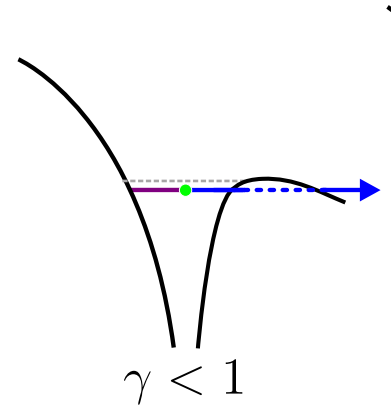

(d) Over-the-barrier Ionization

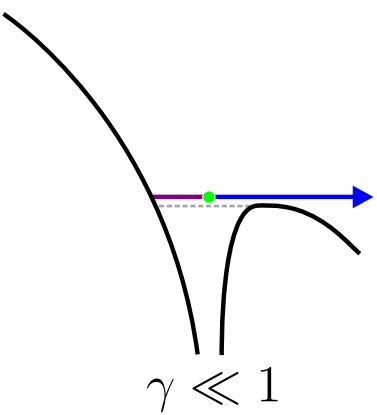

FIG. 1. The atomic Coulomb potentials under the influence of intense laser fields are shown for the (a) field free, (b) multiphoton ionization, (c) tunnel ionization, and (d) over-the-barrier ionization cases. Above-threshold ionization (ATI) is also presented (red vertical arrows) as compared to multi-photon ionization (black vertical arrows). Here, $I_{p}$ is the ionization potential, and $\gamma$ is the Keldysh parameter. Figure adapted from Ref. 198. 


\begin{aligned} \hline Ionization regime & Operating condition \\ \hline Single-photon ionization (SPI) & $\hbar \omega>I_{p} \gg U_{p} \\$ Multi-photon ionization (MPI) & $I_{p}>\hbar \omega \gg U_{p} \\$ Above-threshold ionization (ATI) & $I_{p}>U_{p}>\hbar \omega \\$ Tunnel ionization (TI) & $U_{p}>I_{p}>\hbar \omega\end{aligned}$

TABLE I. The operating conditions in terms of photon energy, $\hbar \omega$, ionization potential, $I_{p}$, and ponderomotive energy, $U_{p}$ are presented for single-photon ionization (SPI), multi-photon ionization (MPI), above-threshold ionization (ATI) and tunnel ionization (TI).

\section{A. Above-Threshold Ionization (ATI)}

Above-threshold ionization (ATI) is an extension of multi-photon ionization where multiple photons are absorbed to not only access the ionization continuum but to surpass the $I_{p}$ by more than one photon, $\hbar \omega$ [34]. In a typical ATI photoelectron spectrum, as shown in Fig. 2, a series of peaks are observed that correspond to each photon absorbed above the $I_{p}$, each of which is separated by a single photon energy, $\hbar \omega$. More strongly, ATI can be observed in the high energy range of the photoelectron spectrum $\left(2 U_{p} \leq E_{r} \leq 10 U_{p}\right)$, referred to as high-order ATI (HATI), where recollision-based strong-field physics can appear, giving rise to elastic and inelastic scattering.

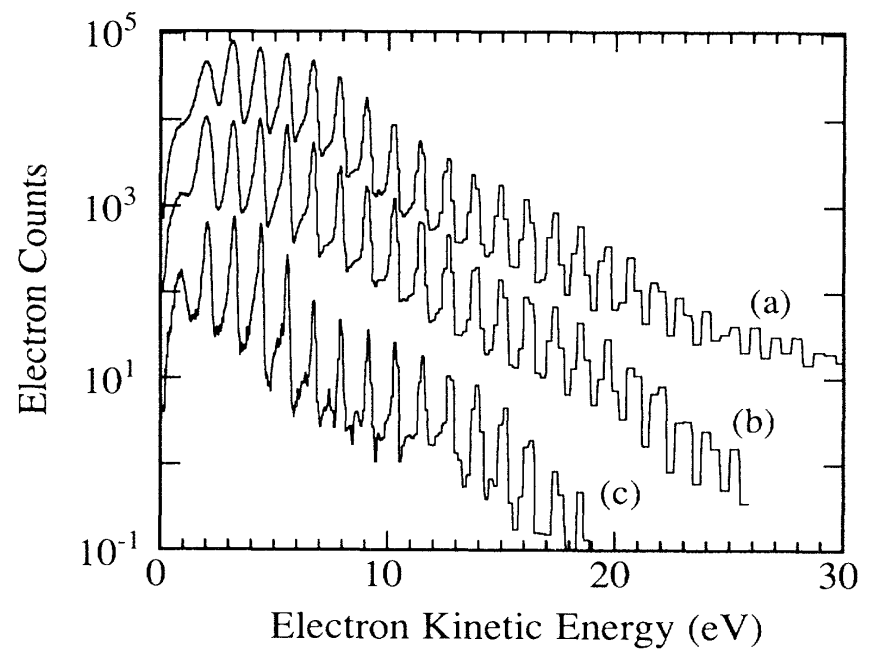

FIG. 2. Above-threshold ionization (ATI) spectrum of xenon illuminated with a $50 \mathrm{ps}, 1.05 \mu \mathrm{m}$ laser pulse at three intensities: (a) $2.0 \times 10^{13} \mathrm{~W} / \mathrm{cm}^{2}$, (b) $1.5 \times 10^{13} \mathrm{~W} / \mathrm{cm}^{2}$, and (c) $1.0 \times 10^{13} \mathrm{~W} / \mathrm{cm}^{2}$. Figure taken from Ref. 78 .

\section{B. High-Harmonic Generation (HHG)}

Attosecond laser pulses of high-photon energies in the extreme ultraviolet (XUV) and X-ray energy region can be produced by high-harmonic generation $(\mathrm{HHG})[6,82,103]$. Pulse trains of attosecond radiation are generated using a multi-cycle femtosecond driving laser pulse, as presented in Fig. 3a with the $27^{\text {th }}-85^{\text {th }}$ harmonics shown [199]. Similarly, a single attosecond pulse with a broadband spectrum can be generated using a near-single cycle driving laser pulse with a continuous broadband spectrum, as shown in Fig. 3b [200], though other so-called 'gating' schemes are also possible [201-205]. The maximum HHG cut-off energy, $E_{\max }$, that can be generated is given by the simpleman's-model-like $E_{\max }=3.17 U_{p}+I_{p}$, and is observed as the abrupt end to the HHG plateau.

These microscopic aspects aside, it is also important to remark that HHG is a macroscopic nonlinear optical process that requires the coherent combination of a large number of emitters to be observed experimentally, and this requires that specific attention be paid to the phase-matching conditions [206], which are often the determining limitation in the production of harmonics. 

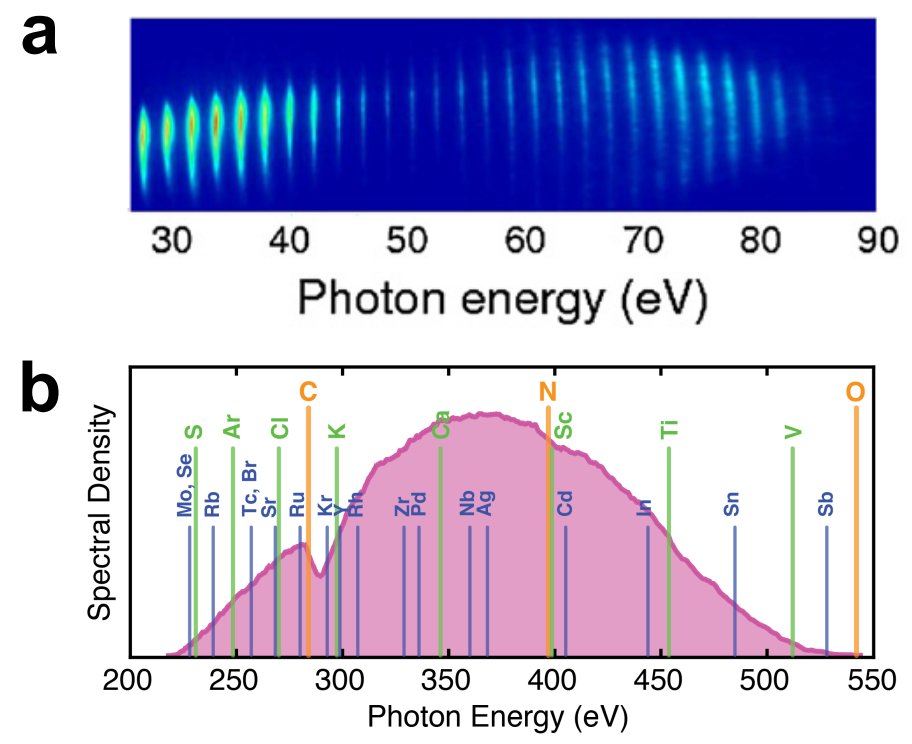

FIG. 3. HHG spectrum for (a) a train of attosecond pulses and (b) an isolated attosecond pulse. In panel (a), argon was illuminated with a $40 \mathrm{fs}$ (10-cycle), $1200 \mathrm{~nm}$ driving laser pulse to generate a spectrum over the $30-90 \mathrm{eV}$ range. In panel (b), helium was ionized by a $12 \mathrm{fs}$ (1.8-cycle), $1850 \mathrm{~nm}$ laser pulse to generate a broadband spectrum over the entire water window range of 284-543 eV, with the K- (orange), L- (green), and M-shell (blue) absorption edges indicated by vertical lines. Figures in panels (a) and (b) were adapted from Refs. 199 and 200, respectively.

\section{Inelastic Scattering: Non-Sequential Double Ionization (NSDI)}

If the recollision of the returning electrion $\left(e_{1}\right)$ with the parent ion is inelastic, then it can transfer enough energy to eject a second electron $\left(e_{2}\right)$. This process is known as non-sequential double ionization (NSDI) [80], and it can proceed via two ionization pathways upon the recollision of $e_{1}$ [207], as shown in Fig. 4a: (i) direct ionization of $e_{2}$ via electron-impact ionization (EII) [103]; or (ii) resonant excitation of $e_{2}$ subsequently followed by its delayed tunnel ionization through recollision-excitation with subsequent ionization (RESI) [208]. A typical signature of NSDI is the correlated detection of two electrons $\left(e_{1}\right.$ and $\left.e_{2}\right)$ in the same emission direction within the two-dimensional momentum map of transverse $\left(p_{\perp}\right)$ and longitudinal $\left(p_{\|}\right)$momenta, as shown in Fig. $4 \mathrm{~b}$.
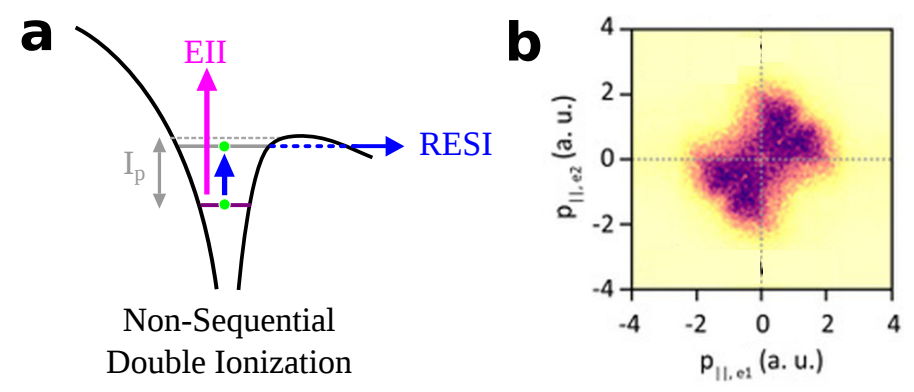

FIG. 4. (a) Non-sequential double ionization (NSDI) illustrated after the recollision of $e_{1}$ with the target ion using an atomic Coulomb potential. After inelastic recollision, either the second electron is ejected through: (i) an electron-impact process (pink arrow) or (ii) excited and subsequently ionized through recollision-excitation with subsequent ionization (RESI; blue arrow). (b) The typical NSDI signature in a two-dimensional momentum map of longitudinal $\left(p_{\|}\right)$and transverse $\left(p_{\perp}\right)$ momenta in atomic units (a.u.) from strong-field ionized $\mathrm{Xe}^{2+}$ ions. Panel (b) was adapted from Ref. [207].

\section{Elastic Scattering: Laser-Induced Electron Diffraction (LIED)}

The highly-energetic returning electron can collide elastically and scatter on the target ion, leading to a momentum transfer between the electron and parent ion. This is known as laser-induced electron diffraction (LIED) [14, 24, 209- 
212] and it can be explained in the framework of laser-driven electron recollision [82, 103]. Structural information is embedded in the photoelectron momentum distribution, appearing as oscillations in the high-energy part of spectrum corresponding to recollision-based physics $\left(2 U_{p} \leq E_{r} \leq 10 U_{p}\right)$ as a function of the emission angle, as shown in Fig. 5 with a zoomed-in view of these oscillations given in the inset. It should be noted that, as opposed to HHG, phase-matching is irrelevant for inelastic and elastic scattering processes (i.e. NSDI and LIED), since the macroscopic observable is an incoherent combination of the emission from the different atoms in the laser focus.

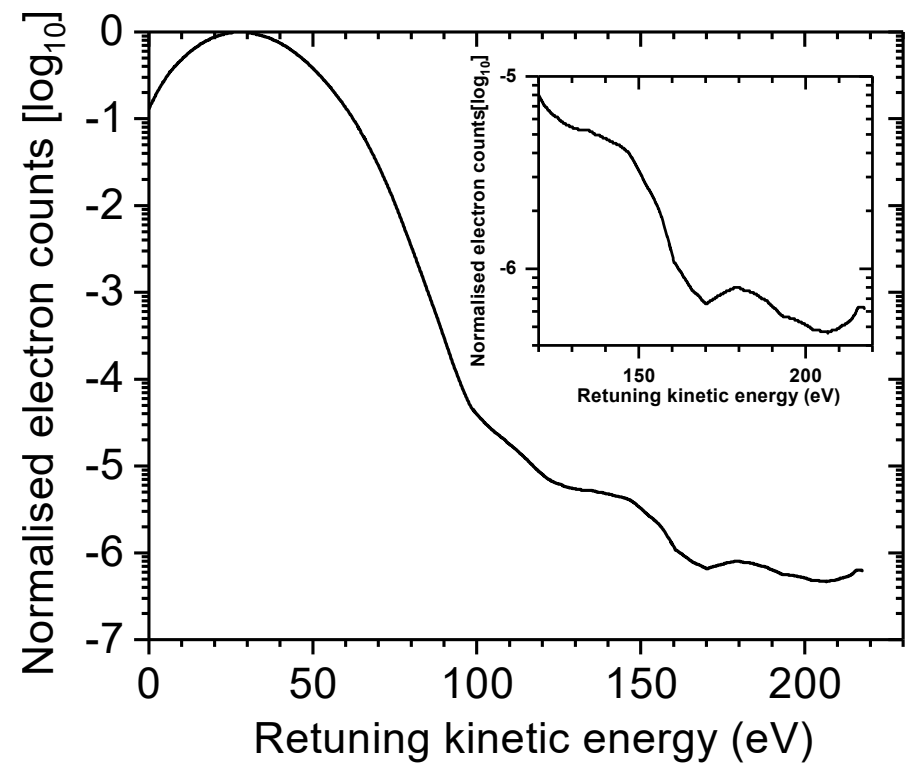

FIG. 5. Typical photoelectron spectrum recorded with LIED of $\mathrm{C}_{2} \mathrm{H}_{2}^{+}$, with the direct photoelectrons (< 3.5 a.u.) and rescattered electrons ( $>3.5$ a.u.) present. Oscillations are clearly seen in the rescattered energy range of the electron signal that is a result of the coherent molecular interference signal which is dependent on the target's geometric structure. The inset shows a zoomed-in view of the oscillations in the differential cross-section of the scattering energy range. Figure adapted from Ref. 210 .

\section{STRONG FIELD APPROXIMATION}

Strictly speaking, neglecting nuclear motion, an atomic or molecular system interacting with a strong electric field pulse is described by the time-dependent Schrödinger equation (TDSE) that captures both the evolution of the (electronic) wave function and the time evolution of the physical observables. The numerical solution of the TDSE offers a full quantum mechanical description of the laser-matter interaction processes; it has been used extensively to study HHG [213-216] and ATI [142, 217-220] in atomic and molecular systems. However, the full numerical integration of the TDSE in all the degrees of freedom of the system is computationally very demanding, when it is at all possible. Moreover, a physical interpretation of the numerical results is highly nontrivial, as always for an ab initio technique. Within this framework, then, approximate methods are welcome, and SFA has consistently been shown over the years to be the workhorse tool for that role.

\section{A. Hamiltonian and TDSE}

Let us consider an atom or molecule under the influence of an intense laser field in the so-called single active electron (SAE) approximation. In the limit when the wavelength of the laser $\lambda_{0}$ is large compared with the Bohr radius, $a_{0}\left(5.29 \times 10^{-11} \mathrm{~m}\right)$, the electric field of the laser beam around the interaction region can be considered spatially homogeneous. Consequently, the interacting atoms will not experience the spatial dependence of the laser electric field and, hence, only its time variation is taken into account - this is the so-called dipole approximation. Note, on the other hand, that certain dynamical effects, even in the long-wavelength limit, can break this approximation [139]. Within this framework, the laser electric field can be written as:

$$
\mathbf{E}(t)=\mathcal{E}_{0} f(t) \sin \left(\omega_{0} t+\phi_{0}\right) \mathbf{e}_{z}
$$


The field of Eq. (3) has a carrier frequency $\omega_{0}=\frac{2 \pi c}{\lambda_{0}}$, where $c$ is the speed of light, and a peak amplitude $\mathcal{E}_{0}$. We consider here that the laser field is linearly polarized along the $z$ direction, with a pulse envelope $f(t)$ and a carrier-envelope phase $\phi_{0}$. More generally, we could consider time-dependent polarization, i.e. replace $\mathcal{E}_{0} \mathbf{e}_{z} \rightarrow \mathbf{E}_{0}(t)$.

The TDSE reads:

$$
i \hbar \frac{\partial}{\partial t}|\Psi(t)\rangle=\hat{H}|\Psi(t)\rangle
$$

where the Hamiltonian, $\hat{H}$, describes the laser-target system in SAE approximation, and is the sum of two terms, i.e.

$$
\hat{H}=\hat{H}_{0}+\hat{U}
$$

where $\hat{H}_{0}$ is the laser-free Hamiltonian of the atomic or molecular system

$$
\hat{H}_{0}=-\frac{\hbar^{2} \nabla^{2}}{2 m}+\hat{V}(\mathbf{r}) \text {, }
$$

with $\hat{V}(\mathbf{r})$ the effective SAE atomic or molecular potential, $m$ the electron mass, and $\hat{U}=-e \mathbf{E}(t) \cdot \mathbf{r}$ the dipole coupling, which describes the interaction of the atomic or molecular system with the laser radiation, written in the length gauge [221, 222] and under the dipole approximation. Note that in atomic units, the electron charge, denoted by $e$, is $e=-1$ a.u., and the Planck constant and electron mass are both set to unity, $\hbar=m=1$ a.u. In this work, however, we keep the explicit constants.

\section{B. "Standard" SFA à la Lewenstein}

We shall restrict ourselves to the regime of low laser frequency and relatively high intensity, where the SFA is expected to be valid [27-29, 33, 55, 107] and to describe well the laser-matter interactions. This corresponds to the tunnelling regime, where the Keldysh parameter $\gamma=\sqrt{I_{p} / 2 U_{p}}$ is less than one, $\gamma<1$ (here $I_{p}$ denotes the ionization potential of the atomic or molecular system, and $U_{p}=\frac{e^{2} \mathcal{E}_{0}^{2}}{4 m \omega_{0}^{2}}$, the ponderomotive energy, i.e. the time-averaged kinetic energy of the oscillations of the electron in the electromagnetic field). In this regime the effects of atomic effective potential on the dynamics of electrons in the continuum are assumed to be small, and they can be treated using perturbation theory. These observations suggest to formulate the "standard SFA" as follows:

(i) The strong field laser does not couple with any bound state beyond the the ground state, $|0\rangle$, so that only it and the continuum (scattering) states, $|\mathbf{p}\rangle$, are taken into account in the dynamics;

(ii) The amplitude of the ground state, $a(t)$, is considered to be known.

(iii) The continuum states are taken from the basis of exact scattering states, which are eigenstates via

$$
\hat{H}_{0}|\mathbf{p}\rangle=\frac{1}{2} \mathbf{p}^{2}|\mathbf{p}\rangle
$$

of the atomic Hamiltonian with a fixed outgoing (kinetic) momentum p. The continuum-continuum matrix selement from $\mathbf{p}$ to $\mathbf{p}^{\prime}$ are then decomposed into their most singular part, proportional to $i \hbar \nabla_{\mathbf{p}} \delta\left(\mathbf{p}-\mathbf{p}^{\prime}\right)$, and the "rest" [29, 55, 107]. The "rest" is then treated in a perturbative manner [29].

The following comments are necessary in order to specify more precisely the above points.

Ad (i) Based on the statement (i), the electronic state $|\Psi(t)\rangle$ that represents the time evolution of the system is a coherent superposition of the ground $|0\rangle$ and the continuum $|\mathbf{p}\rangle$ states [29, 107]:

$$
|\Psi(t)\rangle=e^{i I_{p} t / \hbar}\left(a(t)|0\rangle+\int d^{3} \mathbf{p} b(\mathbf{p}, t)|\mathbf{p}\rangle\right) .
$$

The factor $a(t)$, representing the amplitude of the ground state, is assumed to be known (see below for the ways to evaluate or estimate it). The prefactor $e^{i I_{p} t / \hbar}$ represents the phase oscillations which describe the accumulated electron energy in the ground state $\left(I_{p}=-E_{0}\right.$ is the ionization potential, with $E_{0}$ the ground-state energy of the target system). Furthermore, the transition amplitude to the continuum states is denoted by $b(\mathbf{p}, t)$, and it depends both on the kinetic momentum of the outgoing electron and the laser pulse. Note that if needed other (relevant) bound states may be taken into account in the expression (8) (cf. Refs. 223, 97, 161). 
Ad (ii) There are several ways of evaluating or estimating $a(t)$, depending on the regime of parameters.

- First, one can use ab initio TDSE of the target system to determine the $a(t)$. This is obviously quite costly numerically, but it is much less costly than a full solution of the TDSE which is also required to calculate photoelectron momentum spectra or angular distributions which would need much higher precision, memory and disk storage, and higher computation times.

- Second, one can use any "cheap" approximate method to calculate $a(t)$, such as phase-space averaging or the truncated Wigner approximation [96].

- Third, a broadly-used method is to calculate $a(t)$ analytically using the ionization rates according to the Ammosov-Delone-Krainov theory (ADK rates [44]). To this end, one generalizes these rates to depend on time locally through the time dependence of the laser electric field, which is generally a rather straightforward task (see Appendix A). This approach is valid in the quasi-static regime, when not only the laser frequency, but also the rate of change of the pulse envelope function $f(t)$ are small-meaning that the laser pulse is longer, so that it includes several optical periods.

- Fourth, when the pulse is very short, or it is long but not too strong, there is practically no depletion of the ground state, i.e. $a(t) \simeq 1$. This happens, for instance, for moderately long pulses when the ponderomotive energy is lower than the saturation energy of the system $\left(U_{p}<U_{\text {sat }}\right)$.

- Fifth, one can calculate $a(t)$ within our SFA self-consistently. This approach was already discussed in Ref. 107, but it turned out not to be very precise for the longer pulses - the ADK rates were giving much better agreement with the exact solutions of the TDSE and with the experimental data. This approach seems to be, however, much more adequate and precise for ultrashort, few-cycles pulses. We describe it in detail in Appendix B.

Ad (iii) The continuum-continuum matrix element, independently of the fact whether the effective SAE potential is short-range (as it is for model atoms and negative ions) or Coulomb-like, has the general form:

$$
e\left\langle\mathbf{p}|\mathbf{x}| \mathbf{p}^{\prime}\right\rangle=i e \hbar \nabla_{\mathbf{p}} \delta\left(\mathbf{p}-\mathbf{p}^{\prime}\right)+\hbar \mathbf{g}\left(\mathbf{p}, \mathbf{p}^{\prime}\right)
$$

where the part $\hbar \mathbf{g}\left(\mathbf{p}, \mathbf{p}^{\prime}\right)$ is less singular - typically the strongest singularity it contains corresponds to the onenergy-shell gradient of the Dirac delta of $\mathbf{p}^{2}-\left(\mathbf{p}^{\prime}\right)^{2}$. This part is responsible for rescattering effects in ATI and recollisions in NSMI. Note that since we insist on using the exact scattering states, the dipole matrix element $\langle\mathbf{p}|\mathbf{x}| 0\rangle$ (together with the rescattering continuum-continuum matrix elements) does include the full effects of the effective SAE potential, comprising both the short-range effects as well as any long-range Coulomb effects (if present).

Note also that the SFA in the present formulation (actually equivalent to that of Ref. 29) does not involve plane waves or Volkov solutions! The majority of authors, including ourselves, "erroneously" (in the view of the present formulation) claim that SFA corresponds to the use of Volkov states in the continuum. This is, in principle, false and dangerous. One can use additional approximations, and approximate the exact scattering states by plane waves in the calculations, but this is an additional approximation! It does simplify life and allows one to calculate many things more easily, but it also leads to problems, especially in the case of molecules and other extended targets.

These problems are due to the fact that a plane wave $|\mathbf{p}\rangle$ is not orthogonal to the ground state $|0\rangle$, so that the matrix element $\left\langle\mathbf{p}\left|\mathbf{R}_{0}\right| 0\right\rangle \neq 0$, where $\mathbf{R}_{0}$ is the typical internuclear distance, which is just a constant vector. The lack of orthogonality of $\langle\mathbf{p} \mid 0\rangle \neq 0$ leads to various non-physical and misleading results in applications of, say, "primitive" SFA to molecules (for remedies see Refs. 111, 112). We stress: no remedies are needed, on the other hand, if the exact scattering states are used, since then the orthogonality is assured by construction.

Why, then, do the plane waves and Volkov solutions appear at all? Clearly, this is due to the fact that in the zeroth approximation of SFA we neglect the contribution of $\hbar \mathbf{g}\left(\mathbf{p}, \mathbf{p}^{\prime}\right)$. In this case, the full continuum-continuum matrix element becomes $\left\langle\mathbf{p}|\mathbf{x}| \mathbf{p}^{\prime}\right\rangle=i \hbar \nabla_{\mathbf{p}} \delta\left(\mathbf{p}-\mathbf{p}^{\prime}\right)$, and is exactly equal to that obtained for plane waves and Volkov solutions. That means that the quasi-classical action, describing the propagation of electrons in the continuum, does indeed have a free electron form. For short-range effective potentials this is acceptable, but not for the Coulomb-like ones. That is why the so-called Coulomb corrections are easily included in $\langle\mathbf{p}|\mathbf{x}| 0\rangle$ or $\hbar \mathbf{g}\left(\mathbf{p}, \mathbf{p}^{\prime}\right)$, but much effort has been devoted to find Coulomb corrections to the action- see the Introduction for the relevant references. 


\section{Solutions of the SFA equations}

Our main task in this subsection will be to derive a general expression for the amplitude $b(\mathbf{v}, t)$, which then will be used to calculate ATI spectra and angular distributions, as well as HHG spectra. After some algebra, the time variation of the ground state amplitude, $a(t)$, and the transition amplitude $b(\mathbf{v}, t)$ read:

$$
\begin{aligned}
\dot{a}(t)=- & \frac{i}{\hbar} \int d^{3} \mathbf{p} \mathbf{E}(t) \cdot \mathbf{d}(\mathbf{p}) b(\mathbf{p}, t) \\
\dot{b}(\mathbf{p}, t)=- & \frac{i}{\hbar}\left(\frac{\mathbf{p}^{2}}{2 m}+I_{p}\right) b(\mathbf{p}, t)+\frac{i}{\hbar} \mathbf{E}(t) \cdot \mathbf{d}(\mathbf{p}) a(t) \\
& \quad-e \mathbf{E}(t) \cdot \nabla_{\mathbf{p}} b(\mathbf{p}, t)+i \mathbf{E}(t) \cdot \int d^{3} \mathbf{p}^{\prime} b\left(\mathbf{p}^{\prime}, t\right) \mathbf{g}\left(\mathbf{p}, \mathbf{p}^{\prime}\right) .
\end{aligned}
$$

The first term on the right-hand side of Eq. (10) represents the free phase evolution of the electron in the absence if the oscillating laser field. In the second term we have defined the bound-free transition dipole matrix element as

$$
e\langle\mathbf{p}|\mathbf{r}| 0\rangle=\mathbf{d}(\mathbf{p}) .
$$

Finally, the last two terms describe the continuum-continuum transition, $\nabla_{\mathbf{p}} b(\mathbf{p}, t)$, without the influence of the scattering center, and by considering the core potential, $\int d^{3} e \mathbf{p}^{\prime} b\left(\mathbf{p}^{\prime}, t\right) \mathbf{g}\left(\mathbf{p}, \mathbf{p}^{\prime}\right)$. Here $\mathbf{g}\left(\mathbf{p}, \mathbf{p}^{\prime}\right)$ denotes the rescattering transition matrix element, where the potential core plays an essential role:

$$
e\left\langle\mathbf{p}|\mathbf{x}| \mathbf{p}^{\prime}\right\rangle=i e \hbar \nabla_{\mathbf{p}} \delta\left(\mathbf{p}-\mathbf{p}^{\prime}\right)+\hbar \mathbf{g}\left(\mathbf{p}, \mathbf{p}^{\prime}\right),
$$

Note that (10) is a linear integro-diffential equation for $b(\mathbf{p}, t)$. In the following, we shall describe how it is possible to compute the transition amplitude, $b(\mathbf{p}, t)$, by applying the zeroth and first order perturbation theory to the solution of the partial differential equation Eq. (10). We will split the solution of the transition amplitude, $b(\mathbf{p}, t)$, into two parts: $b_{0}(\mathbf{p}, t)$ and $b_{1}(\mathbf{p}, t)$, i.e. $b(\mathbf{p}, t)=b_{0}(\mathbf{p}, t)+b_{1}(\mathbf{p}, t)$. The zeroth order of our perturbation theory $b_{0}(\mathbf{p}, t)$ will be called the direct term. It describes the transition amplitude for a laser-ionized electron that will never rescatter with the remaining ion. On the other hand, the first-order term, which we call the rescattered term, $b_{1}(\mathbf{p}, t)$, refers to the electrons that, once ionized, will have a certain probability of rescattering with the potential of the parent ion.

\section{Direct-ionization amplitude}

Let us consider the process in which the electron is ionized and does not return to its parent ion. This process is modelled by the direct photoelectron transition amplitude $b_{0}(\mathbf{p}, t)$. As the direct ionization process should have a larger probability compared with the rescattering one [29], one can neglect the last term in Eq. (10). This is what we refer to as the zeroth order solution:

$$
\partial_{t} b_{0}(\mathbf{p}, t)=-\frac{i}{\hbar}\left(\frac{\mathbf{p}^{2}}{2 m}+I_{p}\right) b_{0}(\mathbf{p}, t)+\frac{i}{\hbar} \mathbf{E}(t) \cdot \mathbf{d}(\mathbf{p}) a(t)+\mathbf{E}(t) \cdot \nabla_{\mathbf{p}} b_{0}(\mathbf{p}, t) .
$$

The above equation is a first-order inhomogeneous differential equation, which is easily solved by conventional integration methods (see e.g. Ref. 224). Therefore, the solution can be written as

$$
\begin{array}{rl}
b_{0}(\mathbf{p}, t)=\frac{i}{\hbar} \int_{0}^{t} & d t^{\prime} \mathbf{E}\left(t^{\prime}\right) \cdot \mathbf{d}\left(\mathbf{p}+e \mathbf{A}(t) / c-e \mathbf{A}\left(t^{\prime}\right) / c\right) \\
& \times \exp \left(-i \int_{t^{\prime}}^{t} d t^{\prime \prime}\left[\frac{1}{2 m}\left(\mathbf{p}+e \mathbf{A}(t)-e \mathbf{A}\left(t^{\prime \prime}\right) / c\right)^{2}+I_{p}\right] / \hbar\right) a\left(t^{\prime}\right) .
\end{array}
$$

Here, we have considered that the electron appears in the continuum with kinetic momentum $\mathbf{p}\left(t^{\prime}\right)=\mathbf{p}+e \mathbf{A}(t) / c-$ $e \mathbf{A}\left(t^{\prime}\right) / c$ at the time $t^{\prime}$, where $\mathbf{p}$ is the final kinetic momentum, and $\mathbf{A}(t)=-c \int^{t} \mathbf{E}\left(t^{\prime}\right) d t^{\prime}$ is the vector potential of the electromagnetic field, with $c$ the speed of light. In particular, the vector potential at the time when the electron appears at the continuum $t^{\prime}$ is $\mathbf{A}\left(t^{\prime}\right)$, and at a certain detection time $t$, the vector potential reads $\mathbf{A}(t)$. In addition, it is possible to write Eq. (14) as a function of the canonical momentum $\mathbf{p}_{c}$, defined by $\mathbf{p}_{c}=\mathbf{p}+e \mathbf{A}(t) / c$, and therefore 
the probability transition amplitude for the direct electrons simplifies to [107], where we have eliminated the subscript $c$ :

$$
\begin{array}{rl}
b_{0}(\mathbf{p}, t)=\frac{i}{\hbar} \int_{0}^{t} & d t^{\prime} \mathbf{E}\left(t^{\prime}\right) \cdot \mathbf{d}\left(\mathbf{p}-e \mathbf{A}\left(t^{\prime}\right) / c\right) a\left(t^{\prime}\right) \\
& \times \exp \left(-i \int_{t^{\prime}}^{t} d \tilde{t}\left[\frac{1}{2 m}(\mathbf{p}-e \mathbf{A}(\tilde{t}) / c)^{2}+I_{p}\right] / \hbar\right) .
\end{array}
$$

This expression is understood as the sum of all the ionization events which occur from the time $t^{\prime}$ to $t$ [137]. Then, the instantaneous transition probability amplitude of an electron at a time $t^{\prime}$, at which it appears into the continuum with momentum $\mathbf{p}\left(t^{\prime}\right)=\mathbf{p}-e \mathbf{A}\left(t^{\prime}\right) / c$, is defined by the argument of the integral in Eq. (15). Furthermore, the exponent phase factor in Eq. (15) denotes the "semi-classical action", $S\left(\mathbf{p}, t, t^{\prime}\right)$, that defines a possible electron trajectory from the birth time $t^{\prime}$ until the "detection" time $t$ [29]:

$$
S\left(\mathbf{p}, t, t^{\prime}\right)=\int_{t^{\prime}}^{t} d \tilde{t}\left[\frac{1}{2 m}(\mathbf{p}-e \mathbf{A}(\tilde{t}) / c)^{2}+I_{p}\right]
$$

As our purpose is to obtain the final transition amplitude $b_{0}(\mathbf{p}, t)$, the time $t$ will be fixed at the end of the laser field, $t=t_{\mathrm{F}}$. For our calculations, we thus define the integration time window as $t \in\left[0, t_{\mathrm{F}}\right]$. Therefore, we set $\mathbf{E}(0)=\mathbf{E}\left(t_{\mathrm{F}}\right)=\mathbf{0}$, in such a way to make sure that the electromagnetic field is a time oscillating wave and does not have static components. The same arguments are applied to the vector potential $\mathbf{A}(t)$. In concrete calculations we have defined the laser pulse envelope as $f(t)=\sin ^{2}\left(\frac{\omega_{0} t}{2 N_{c}}\right)$ where $N_{c}$ denotes the number of total cycles.

Note that, for an arbitrary electromagnetic field, it is possible for $\mathbf{A}_{\mathrm{F}}(t) \neq 0$, i.e. vector potential does not necessarily vanish at the end of the pulse, implying that the kinetic momentum at $t_{\mathrm{F}}$ is $\mathbf{p}_{\text {kin }}=\mathbf{p}-e \mathbf{A}\left(t_{\mathrm{F}}\right) / c$; if that is the case then it should be considered carefully, since it is $\mathbf{p}_{\text {kin }}$ which is detected in experiments. However, for laser pulses that are focused away from their source and in the paraxial approximation, nonzero-area pulses of this form are not possible, and the vector potential can be taken as zero on both sides of the pulse.

\section{E. Rescattering transition amplitude}

In order to find a solution for the transition amplitude of the rescattered photoelectrons, $b_{1}(\mathbf{p}, t)$, we have considered the rescattering core matrix element $\mathbf{g}\left(\mathbf{p}, \mathbf{p}^{\prime}\right)$ term of Eq. (10) different than zero, i.e. $\mathbf{g}\left(\mathbf{p}, \mathbf{p}^{\prime}\right) \neq \mathbf{0}$. In addition, the first-order perturbation theory is applied to obtain $b_{1}(\mathbf{p}, t)$ by inserting the zeroth-order solution $b_{0}(\mathbf{p}, t)$ in the righthand side of Eq. (10). Then, we obtain $b_{1}(\mathbf{p}, t)$ as a function of the canonical momentum p (neglecting the subscript c) as follows:

$$
\begin{aligned}
b_{1}(\mathbf{p}, t)=\left(\frac{i}{\hbar}\right)^{2} & \int_{0}^{t} d t^{\prime} \exp \left[-i S\left(\mathbf{p}, t, t^{\prime}\right) / \hbar\right] \mathbf{E}\left(t^{\prime}\right) \cdot \int_{0}^{t^{\prime}} d t^{\prime \prime} \int d^{3} \mathbf{p}^{\prime} \mathbf{g}\left(\mathbf{p}-e \mathbf{A}\left(t^{\prime}\right) / c, \mathbf{p}^{\prime}-e \mathbf{A}\left(t^{\prime}\right) / c\right) \\
& \times \mathbf{E}\left(t^{\prime \prime}\right) \cdot \mathbf{d}\left(\mathbf{p}^{\prime}-e \mathbf{A}\left(t^{\prime \prime}\right) / c\right) a\left(t^{\prime \prime}\right) \exp \left[-i S\left(\mathbf{p}^{\prime}, t^{\prime}, t^{\prime \prime}\right) / \hbar\right]
\end{aligned}
$$

This last equation contains all the information about the rescattering process. In particular, it is referred to the probability amplitude of an emitted electron at the time $t^{\prime \prime}$, with an amplitude given by

$$
\mathbf{E}\left(t^{\prime \prime}\right) \cdot \mathbf{d}\left(\mathbf{p}^{\prime}-e \mathbf{A}\left(t^{\prime \prime}\right) / c\right) a\left(t^{\prime \prime}\right)
$$

In this step the electron has a kinetic momentum of $\mathbf{v}^{\prime}\left(t^{\prime \prime}\right)=\mathbf{p}^{\prime}-e \mathbf{A}\left(t^{\prime \prime}\right) / c$. The last factor, $\exp \left[-i S\left(\mathbf{p}^{\prime}, t^{\prime}, t^{\prime \prime}\right)\right]$, is the accumulated phase of an electron born at the time $t^{\prime \prime}$ until it rescatters at time $t^{\prime}$. The intervening term, $\mathbf{g}\left(\mathbf{p}-e \mathbf{A}\left(t^{\prime}\right) / c, \mathbf{p}^{\prime}-e \mathbf{A}\left(t^{\prime}\right) / c\right)$, contains the structural matrix element of the continuum-continuum transition at the re-scattering time $t^{\prime}$. At this particular moment in time, the electron changes its kinetic momentum from $\mathbf{p}^{\prime}-e \mathbf{A}\left(t^{\prime}\right) / c$ to $\mathbf{p}-e \mathbf{A}\left(t^{\prime}\right) / c$. We stress, however, that the term $\mathbf{g}\left(\mathbf{v}, \mathbf{v}^{\prime}\right)$ does not necessarily imply that the electron returns to the ion core.

In addition to this, the phase term $\exp \left[-i S\left(\mathbf{p}, t, t^{\prime}\right)\right]$ defines the accumulated phase of the electron after the rescattering from the time $t^{\prime}$ to the "final" one $t$ when the electron is "measured" at the detector with momentum p. In particular, note that the photoelectron spectrum, $\left|b\left(\mathbf{p}, t_{\mathrm{F}}\right)\right|^{2}$, is a coherent superposition of both solutions, $b_{0}\left(\mathbf{p}, t_{\mathrm{F}}\right)$ and $b_{1}\left(\mathbf{p}, t_{\mathrm{F}}\right)$, together with an interference term:

$$
\begin{aligned}
\left|b\left(\mathbf{p}, t_{\mathrm{F}}\right)\right|^{2} & =\left|b_{0}\left(\mathbf{p}, t_{\mathrm{F}}\right)+b_{1}\left(\mathbf{p}, t_{\mathrm{F}}\right)\right|^{2} \\
& =\left|b_{0}\left(\mathbf{p}, t_{\mathrm{F}}\right)\right|^{2}+\left|b_{1}\left(\mathbf{p}, t_{\mathrm{F}}\right)\right|^{2}+b_{0}\left(\mathbf{p}, t_{\mathrm{F}}\right) b_{1}^{*}\left(\mathbf{p}, t_{\mathrm{F}}\right)+\text { c.c. }
\end{aligned}
$$


So far we have formulated a model, which describes the photoionization process leading to two main terms, namely, a direct $b_{0}\left(\mathbf{p}, t_{\mathrm{F}}\right)$ and a rescattering $b_{1}\left(\mathbf{p}, t_{\mathrm{F}}\right)$ one. As the complex transition amplitude, Eq. (15), is a single time integral, it can be integrated numerically without major problems. However, the multiple time (" $2 \mathrm{D}$ ") and momentum ("3D") integrals of the re-scattering term, Eq. (17), present an increasingly difficult and demanding task from a computational perspective. In order to reduce the computational difficulties, and to obtain a physical meaning of the ATI process, one may employ saddle-point methods to evaluate these highly-oscillatory integrals.

The main challenge to calculate the ATI spectrum is then the computation of the bound-free transition dipole matrix element, $\mathbf{d}(\mathbf{p})$, and the continuum-continuum transition re-scattering matrix element $\mathbf{g}\left(\mathbf{p}, \mathbf{p}^{\prime}\right)$ for a given atomic or molecular system. In the Appendices, we discuss how to do this analytically for a model atom or molecule with a short-range separable potential.

\section{F. Time-dependent dipole moment}

Finally, to analyse the HHG we need to know the electron acceleration, or at least the time dependent electron dipole moment. This is dominantly given by the zeroth order solution of the SFA equations. It is then given by the dynamical version of the celebrated Landau-Dykhne formula,

$$
\begin{aligned}
\langle\mathbf{x}(t)\rangle=\operatorname{Re}\left[\frac{i}{\hbar}\right. & \int_{0}^{t} d t^{\prime} \int d^{3} \mathbf{p} a^{*}(t) \mathbf{d}(\mathbf{p}-e \mathbf{A}(t) / c) \mathbf{E}\left(t^{\prime}\right) \cdot \mathbf{d}\left(\mathbf{p}-e \mathbf{A}\left(t^{\prime}\right) / c\right) a\left(t^{\prime}\right) \\
& \left.\times \exp \left(-i \int_{t^{\prime}}^{t} d \tilde{t}\left[\frac{1}{2 m}(\mathbf{p}-e \mathbf{A}(\tilde{t}) / c)^{2}+I_{p}\right] / \hbar\right)\right]
\end{aligned}
$$

which is then generally compared to experiment via its frequency-domain version, the Fourier transform

$$
\begin{aligned}
\tilde{\mathbf{x}}(\Omega)= & \int_{-\infty}^{\infty}\langle\mathbf{x}(t)\rangle e^{+i \Omega t} d t \\
= & \operatorname{Re}\left[\frac{i}{\hbar} \int_{-\infty}^{\infty} d t \int_{0}^{t} d t^{\prime} \int d^{3} \mathbf{p} a^{*}(t) \mathbf{d}(\mathbf{p}-e \mathbf{A}(t) / c) \mathbf{E}\left(t^{\prime}\right) \cdot \mathbf{d}\left(\mathbf{p}-e \mathbf{A}\left(t^{\prime}\right) / c\right) a\left(t^{\prime}\right)\right. \\
& \left.\quad \times \exp \left(-i \int_{t^{\prime}}^{t} d \tilde{t}\left[\frac{1}{2 m}(\mathbf{p}-e \mathbf{A}(\tilde{t}) / c)^{2}+I_{p}\right] / \hbar+i \Omega t\right)\right],
\end{aligned}
$$

where $\Omega$ is the frequency of the emitted harmonic.

\section{G. Saddle-point methods and quantum orbits}

The SFA results as we have obtained them thus far, i.e. Eqs. (15) and (17) for the direct- and rescattered-electron momentum wavefunctions and Eq. (24) for the frequency-domain harmonic dipole, in what is known as their timeintegrated versions with explicit integrals over the times of ionization and recollision or recombination, are often perfectly sufficient for the evaluation of the relevant physical observables via a direct numerical integration. However, they generally involve the integration of highly oscillatory terms, such as those contained in the phase factor of the harmonic dipole in (24),

$$
\exp \left(-i S_{\Omega}\left(\mathbf{p}, t, t^{\prime}\right) / \hbar\right)=\exp \left(-\frac{i}{\hbar} \int_{t^{\prime}}^{t}\left[\frac{1}{2 m}(\mathbf{p}-e \mathbf{A}(\tilde{t}) / c)^{2}+I_{p}\right] d \tilde{t}+i \Omega t\right),
$$

where the phase of the exponential can vary wildly, introducing extreme cancellations in the integrand that require increased precision in the numerical integration to calculate correctly. Generally speaking, the phase in this factor can be estimated by considering its scaling once the pulse amplitude $\mathcal{E}_{0}$ and frequency $\omega$ are factored out by dedimensionalizing the integral in the exponent, giving contributions which scale with the so-called strong-field parameter $z=U_{p} / \omega$ and with $I_{p} / \omega$. For experiments with a strong low-frequency field, both of these parameters are large, and the exponent in Eq. (25) will quickly cover many radians without giving the rest of the integrand time to change, giving rise to cancellations in the integral.

As mentioned earlier, this problem can be overcome by approximating any relevant oscillatory integrals using the method of steepest descents, which approximates the integrals using the values of the integrand at stationary points of the action - in exactly the same way as the classical trajectories emerge as the stationary-action points of the 
Feynman path integral [137]. Using the paradigmatic case of HHG as an example, this requires us to find solutions to the stationary-point equations over the three integration variables,

$$
\begin{aligned}
\frac{\partial}{\partial t^{\prime}} S_{\Omega}\left(\mathbf{p}, t, t^{\prime}\right) & =\frac{1}{2 m}\left(\mathbf{p}-e \mathbf{A}\left(t^{\prime}\right) / c\right)^{2}+I_{p}=0 \\
\frac{\partial}{\partial t} S_{\Omega}\left(\mathbf{p}, t, t^{\prime}\right)+\Omega & =\frac{1}{2 m}(\mathbf{p}-e \mathbf{A}(t) / c)^{2}+I_{p}=\Omega \\
\nabla_{\mathbf{p}} S_{\Omega}\left(\mathbf{p}, t, t^{\prime}\right) & =\frac{1}{m} \int_{t^{\prime}}^{t}[\mathbf{p}-e \mathbf{A}(\tilde{t}) / c] d \tilde{t}=0
\end{aligned}
$$

which are often termed the tunnelling, recollision, and return equations, respectively. (For other phenomena, these should be adjusted accordingly, by e.g. dropping $(27,28)$ for the direct-electron ionization amplitude.) The tunnelling equation here, Eq. (26), is the central, determining structure, both because of its prevalence over all SFA applications and because both of the terms on its left-hand side, $\frac{1}{2}\left(\mathbf{p}-e \mathbf{A}\left(t^{\prime}\right) / c\right)^{2}$ and $I_{p}$, are ostensibly positive (for real $t^{\prime}$ ), which means that solutions will only be possible if $t^{\prime}$ (and, with it, all the other variables) are complex-valued.

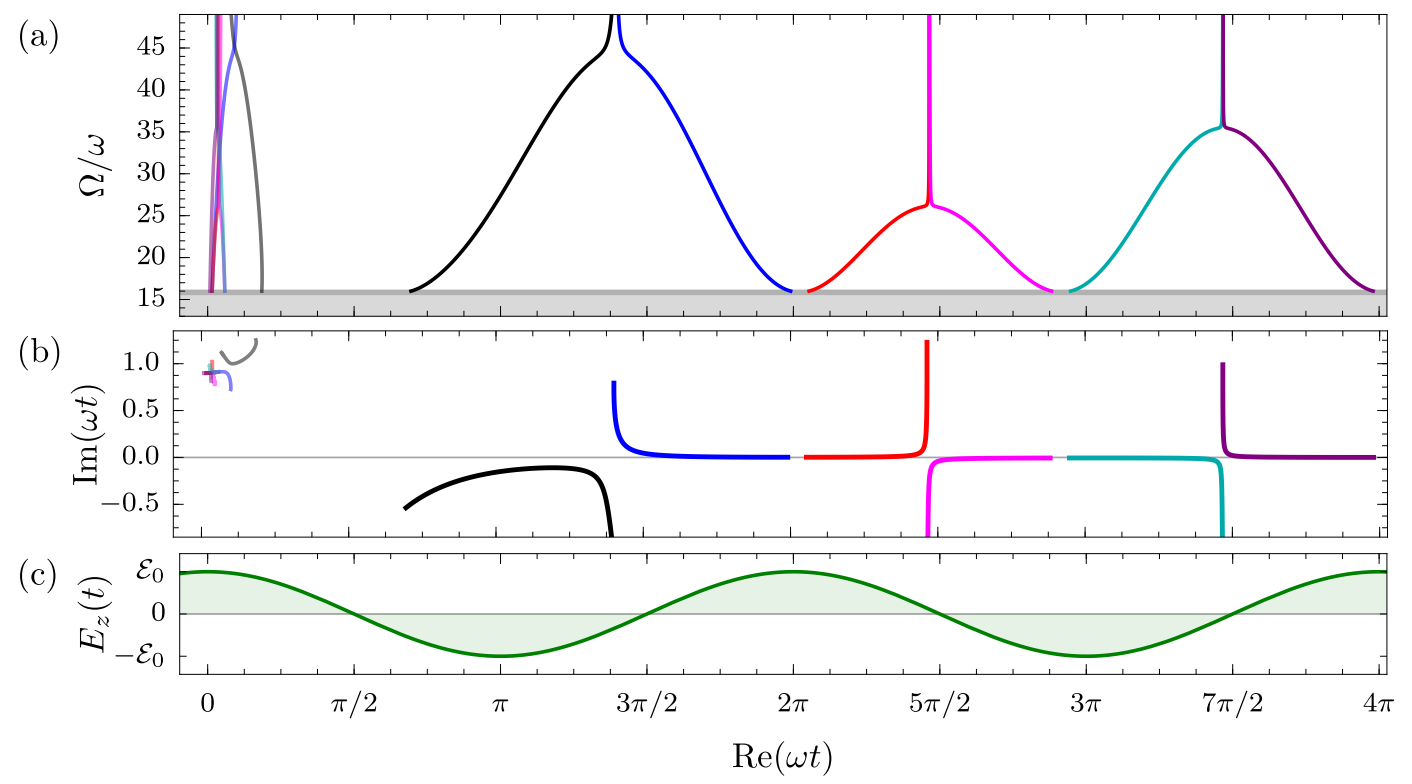

FIG. 6. Saddle-point trajectories for HHG produced by helium in a monochromatic field of wavelength $800 \mathrm{~nm}$ and intensity $2 \times 10^{14} \mathrm{~W} / \mathrm{cm}^{2}$. The recollision and ionization saddle points (shown in solid and faint lines in (b)) form a series of curves in complex time, with close-to-real recollision times in the harmonic plateau that then veer off into imaginary time at the cutoff. When plotted as an energy-time relationship (or, more precisely, as the harmonic order $\Omega / \omega$ versus the recollision time, shown in (a)), the saddle-point curves 'wrap' around the simple-man model's purely classical relationships. We show the electric field in use in (c) for reference. The below-threshold region, which is not well described by the SFA, is shown shaded in (a).

Within that steepest-descent approximation, then, SFA amplitudes are given by a sum over all the relevant saddlepoint roots that contribute to the deformed integration contour,

$$
\tilde{\mathbf{x}}(\Omega)=\operatorname{Re}\left[i \sum_{s} H\left(t_{s}, t_{s}^{\prime}, \mathbf{p}_{s}\right) a^{*}\left(t_{s}\right) \mathbf{d}\left(\mathbf{p}_{s}-e \mathbf{A}\left(t_{s}\right) / c\right) \mathbf{E}\left(t_{s}^{\prime}\right) \cdot \mathbf{d}\left(\mathbf{p}_{s}-e \mathbf{A}\left(t_{s}^{\prime}\right) / c\right) a\left(t_{s}^{\prime}\right) e^{-i S_{\Omega}\left(\mathbf{p}_{s}, t_{s}, t_{s}^{\prime}\right) / \hbar}\right] .
$$

with an additional Hessian factor $H\left(t_{s}, t_{s}^{\prime}, \mathbf{p}_{s}\right)$ that accounts for the width of the complex-integration gaussians being approximated [225]. (Alternatively, it is also possible to perform a partial saddle-point approximation over momentum only, keeping the unique root of the return equation (28) $\mathbf{p}_{s}=\mathbf{p}_{s}\left(t, t^{\prime}\right)$ as a function of the ionization and recollision times, and then integrate numerically.) For the full saddle-point method, the ionization time typically has a large imaginary part and it is confined to a small window shortly after the peak of the field, while the recollision time comes in a series of so-called quantum orbits that span the following periods, as shown in Fig. 6.

Typically, the only quantum orbits that contribute significantly to harmonic generation are the so-called short and long trajectories, shown in black and blue (resp.) in Fig. 6(b). The long trajectories, which ionize closer to the peak of the field, have a higher single-atom harmonic yield, but the phase-matching conditions are typically chosen to 
select the contribution of the short trajectories, which are easier to phase-match. The higher-order returns, which recollide more than one period after the ionization, typically spend too much time in the continuum accumulating an intensity-dependent phase for them to form a macroscopically-coherent emission, but under dedicated circumstances it is still possible to observe signatures of their presence [157, 158].

\section{H. Polarization effects}

The above analysis of quantum orbits becomes, obviously, more complex when the laser fields have more complicated patterns of polarization beyond the simple linear one. Elliptical polarization was considered in the context of HHG already in the pioneering papers by P. Corkum [103]: the electron trajectories in such situation form ellipses, and essentially miss the parent ion, leading to a rapid decrease of the HHG efficiency with increasing ellipticity. These trajectory-based predictions were first confirmed in experiments by Budil et al. [226], and they can be used to produce 'gating' schemes [201-203] to produce isolated attosecond pulses by using a time-dependent polarization that changes across the pulse from circular to linear and back.

The late Bertrand Carré was also among the pioneers of polarization studies in HHG. The first experimental results of ellipticity dependence of the harmonic yield were published in Ref. 227, in which the detailed SFA theory of HHG by an elliptically polarized laser field was investigated. The following seminal paper [228] was the first one to discuss measurements of the (partial) polarization of high harmonics generated by elliptically polarized laser fields, with careful comparison to SFA-based theory including propagation. This paper stimulated many researchers to search for ways to control polarization of harmonics. The Holy Grail was to generate HH with left- and right-circular polarization to be able to use them to study circular dichroism in absorption (to distinguish, for instance, chiral molecules), or to study chiral effects in magnetism.

The rapid decrease of HHG efficiency with ellipticity suggested looking for scenarios, in which linear polarization is used. Pioneering ideas were formulated by P.-M. Paul in his doctoral thesis [133], and developed further in the group of B. Carré by Y. Mairesse, employing first two-photon absorption of one XUV and one IR photon, and later resonant HHG [229, 230], and also HHG generated by linearly-polarized light pulses applied to aligned molecules [231-233].

A breakthrough method was proposed by D. B. Milošević [234] and implemented by O. Cohen, using two circularlypolarized beams with a frequency ratio of 1:2 and opposite helicity [235], which permits the generation of bright phase-matched circularly-polarized extreme ultraviolet high harmonics [236; for a review see 237]. The original method and results of Ref. 228 was developed further to completely characterize the state of elliptically-polarized light by electron-ion vector correlations [238], and finally to realise the complete polarimetry of high harmonics [239]. These methods have recently been applied to HHG generated under O. Cohen's 'bicircular' fields [240], providing a clear evidence for depolarization of high harmonics.

It is worth mentioning that more laser fields with "exotic" polarization (spin) and orbital angular momentum have been proposed recently (see Ref. 241 and references therein). These so-called polarization torus knots, proposed in Ref. 242, when applied to atoms in a form of ultrashort and ultraintense pulse, generate "exotic" harmonics that conserve torus-knot angular momentum, a topologically-nontrivial mixture between spin and orbital angular momentum.

One should also say that ellipticity and polarization effects play an important role in ATI and multielectron ionization. A nice example of the ATI results is included in the Science paper of 2001 [137], where G. Paulus was able to characterize a whole plethora of trajectories corresponding to rescattering of electrons in elliptically polarized laser fields. In the same paper, Carré and Salières present spectra of high harmonics that allow one to identify directly the contribution of the "short" and "long" electronic trajectories.

\section{TWO-ELECTRON PROCESSES}

\section{A. Hamiltonian and states}

In order to describe higher order processes we must extended this formalism to include more active electrons. We start by formulating the SFA equations for two active electrons, this allows us to model higher order strong field ionization process such as non-sequential double ionization (NSDI). We also include excited states in the wave function to allow for the recollision excitation with subsequent ionization (RESI) pathway of NSDI; otherwise only the the direct electron impact ionization (EII) pathway would be present. Following a similar procedure to the one electron 
case, the ansatz for the wavefunction can be written as

$$
|\psi(t)\rangle=\exp \left(i E_{0} t / \hbar\right)\left(a(t)|0\rangle+\int d^{3} \mathbf{p} b(\mathbf{p}, t)|\mathbf{p}, 0\rangle+\sum_{\eta 0} \int d^{3} \mathbf{p} c(\mathbf{p}, \eta, t)|\mathbf{p}, \eta\rangle+\iint d^{3} \mathbf{p} d^{3} \mathbf{p}^{\prime} d\left(\mathbf{p}, \mathbf{p}^{\prime}, t\right)\left|\mathbf{p}, \mathbf{p}^{\prime}\right\rangle\right)
$$

where $|0\rangle$ is the two electron ground state, $|\mathbf{p}, 0\rangle$ gives the two electron state, where the first electron has been promoted to a continuum scattering state with momentum $\mathbf{p},|\mathbf{p}, \eta\rangle$ is similar but the second electron is in an excited state with a principle quantum number $\eta$ while, $\left|\mathbf{p}, \mathbf{p}^{\prime}\right\rangle$ denotes both electron in continuum scattering states. The state $|\eta, 0\rangle$ could be included to allow for some additional effects such as single electron frustrated tunnelling, but we will neglect it for now as we are interested in two electron effects, where this state will play almost no role. These states are all eigenstates of the two particle Hamiltonian

$$
\hat{H}_{0}=\sum_{i=1}^{2}\left(\frac{\hat{\mathbf{p}}_{i}^{2}}{2 m}+V\left(\mathbf{r}_{i}\right)\right)+V\left(\mathbf{r}_{1}-\mathbf{r}_{2}\right)
$$

where $\hat{\mathbf{p}}_{i}$ are single particle momentum operators, $V\left(\mathbf{r}_{i}\right)$ gives the interaction of each particle with the atomic/ molecular core and $V\left(\mathbf{r}_{1}-\mathbf{r}_{2}\right)$ gives the interaction between the two electrons. Note that including the interaction between electrons means that none of the two particle states introduced above can be written as products of one particle states, e.g. $|\mathbf{p}, 0\rangle \neq|\mathbf{p}\rangle|0\rangle$. However, we can write down the energy eigenvalue equations for each

$$
\begin{array}{rlrl}
\hat{H}_{0}|0\rangle & =-E_{0}|0\rangle, & E_{0} & =I_{2 p} \text { (two-electron ionization potential); } \\
\hat{H}_{0}|\mathbf{p}, 0\rangle & =\left(\frac{\hbar p^{2}}{2 m}-E_{10}\right)|\mathbf{p}, 0\rangle, & E_{10}=I_{1 p} \text { (one-electron ionization potential); } \\
\hat{H}_{0}|\mathbf{p}, \eta\rangle & =\left(\frac{\hbar p^{2}}{2 m}-E_{1 \eta}\right)|\mathbf{p}, \eta\rangle, & E_{1 \eta}=I_{1 \eta, p} \text { (one-electron excited-state ionization potential); } \\
\hat{H}_{0}\left|\mathbf{p}, \mathbf{p}^{\prime}\right\rangle & =\left(\frac{\hbar p^{2}}{2 m}+\frac{\hbar p^{\prime 2}}{2 m}\right)\left|\mathbf{p}, \mathbf{p}^{\prime}\right\rangle, &
\end{array}
$$

Given that we are accounting for electron correlation, $E_{0}$ will generally be different from $2 E_{10}$; however, often the correlation is weak and then this is a good approximation to make. If one assumes non-interacting electrons, this amounts to dropping the last term in Eq. (31), then the following substituting can be made for each of the two particle states

$$
\begin{aligned}
|0\rangle \rightarrow\left|\psi_{0}\right\rangle & =|0\rangle|0\rangle \\
|\mathbf{p}, 0\rangle \rightarrow\left|\psi_{0}(\mathbf{p})\right\rangle & =\frac{1}{\sqrt{2}}(|\mathbf{p}\rangle|0\rangle+|\mathbf{p}\rangle|0\rangle), \\
|\mathbf{p}, \eta\rangle \rightarrow\left|\psi_{0}(\mathbf{p}, \eta)\right\rangle & =\frac{1}{\sqrt{2}}(|\mathbf{p}\rangle|\eta\rangle+|\mathbf{p}\rangle|\eta\rangle), \\
\left|\mathbf{p}, \mathbf{p}^{\prime}\right\rangle \rightarrow\left|\psi_{0}\left(\mathbf{p}, \mathbf{p}^{\prime}\right)\right\rangle & =\frac{1}{\sqrt{2}}\left(|\mathbf{p}\rangle\left|\mathbf{p}^{\prime}\right\rangle+|\mathbf{p}\rangle\left|\mathbf{p}^{\prime}\right\rangle\right) .
\end{aligned}
$$

Note we do not use anti-symmetric superpositions as we consider two electrons from a singlet spin state, so that the spins will already be anti-symmetric. These will be eigenstates of the Hamiltonian $\hat{H}_{0}$ without the electron-electron interaction term.

As in the one particle case the full Hamiltonian is given by laser-free and dipole coupling Hamiltonians as in Eq. (5) but now in the two particle case the dipole coupling is given by

$$
\hat{U}=-\sum_{i=1}^{2} e \mathbf{E}(t) \cdot \mathbf{r}_{i}=-\mathbf{E}(t) \cdot e\left(\hat{\mathbf{r}}_{1}+\hat{\mathbf{r}}_{2}\right)
$$

We will proceed as before and derive the integro-differential equations for $a(t), b(\mathbf{p}, t), c(\mathbf{p}, \eta, t)$ and $d\left(\mathbf{p}, \mathbf{p}^{\prime}, t\right)$. However, first we will introduce the dipole matrix elements required for each possible kind of transition between the two particle states. The matrix elements will follow the convention that the left state will have a lower or equal energy 


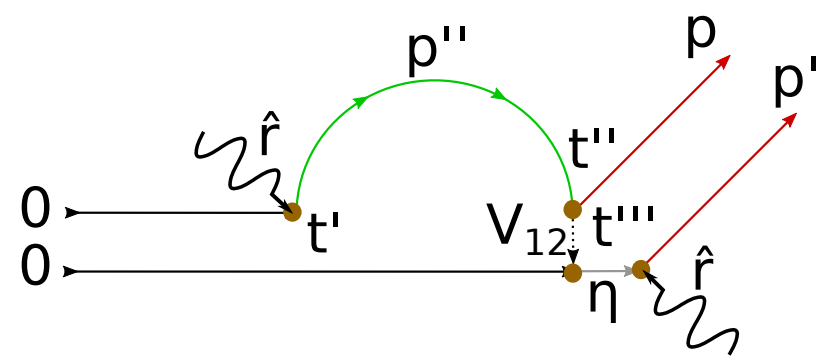

FIG. 7. The complete RESI process showing all three transitions. The three nodes marked at the times $t^{\prime}, t^{\prime \prime}$ and $t^{\prime \prime \prime}$ show the three transitions identified in the chain in Eq. (42).

to the right hand state. Then the dipole matrix elements can be defined in the following way

$$
\begin{array}{rlrl}
\mathbf{d}(\mathbf{p}) & :=\left\langle 0\left|e\left(\hat{\mathbf{r}}_{1}+\hat{\mathbf{r}}_{2}\right)\right| \mathbf{p}, 0\right\rangle, & \mathbf{g}\left(\mathbf{p}, \mathbf{p}^{\prime}\right) & :=\left\langle\mathbf{p}, 0\left|e\left(\hat{\mathbf{r}}_{1}+\hat{\mathbf{r}}_{2}\right)\right| \mathbf{p}^{\prime}, 0\right\rangle, \\
\mathbf{d}(\mathbf{p}, \eta) & :=\left\langle 0\left|e\left(\hat{\mathbf{r}}_{1}+\hat{\mathbf{r}}_{2}\right)\right| \mathbf{p}, \eta\right\rangle, & \mathbf{g}\left(\mathbf{p}, \mathbf{p}^{\prime}, \eta\right) & :=\left\langle\mathbf{p}, 0\left|e\left(\hat{\mathbf{r}}_{1}+\hat{\mathbf{r}}_{2}\right)\right| \mathbf{p}^{\prime}, \eta\right\rangle, \\
\mathbf{d}\left(\mathbf{p}, \mathbf{p}^{\prime}\right) & :=\left\langle 0\left|e\left(\hat{\mathbf{r}}_{1}+\hat{\mathbf{r}}_{2}\right)\right| \mathbf{p}, \mathbf{p}^{\prime}\right\rangle & \mathbf{g}\left(\mathbf{p}, \mathbf{p}^{\prime}, \mathbf{p}^{\prime \prime}\right) & :=\left\langle\mathbf{p}, 0\left|e\left(\hat{\mathbf{r}}_{1}+\hat{\mathbf{r}}_{2}\right)\right| \mathbf{p}^{\prime}, \mathbf{p}^{\prime \prime}\right\rangle, \\
\mathbf{h}\left(\mathbf{p}, \eta, \mathbf{p}^{\prime}, \eta^{\prime}\right) & :=\left\langle\mathbf{p}, \eta\left|e\left(\hat{\mathbf{r}}_{1}+\hat{\mathbf{r}}_{2}\right)\right| \mathbf{p}^{\prime}, \eta^{\prime}\right\rangle, & \\
\mathbf{h}\left(\mathbf{p}, \eta, \mathbf{p}^{\prime}, \mathbf{p}^{\prime \prime}\right) & :=\left\langle\mathbf{p}, \eta\left|e\left(\hat{\mathbf{r}}_{1}+\hat{\mathbf{r}}_{2}\right)\right| \mathbf{p}^{\prime}, \mathbf{p}^{\prime \prime}\right\rangle, & \mathbf{i}\left(\mathbf{p}, \mathbf{p}^{\prime}, \mathbf{p}^{\prime \prime}, \mathbf{p}^{\prime \prime \prime}\right) & :=\left\langle\mathbf{p}, \mathbf{p}^{\prime}\left|e\left(\hat{\mathbf{r}}_{1}+\hat{\mathbf{r}}_{2}\right)\right| \mathbf{p}^{\prime \prime}, \mathbf{p}^{\prime \prime \prime}\right\rangle .
\end{array}
$$

Note that, due to symmetry, the dipole matrix element from $|0\rangle$ to $|0\rangle$ will be zero. Each matrix element has an important physical meaning, which we will discuss in some detail for both the interacting and non-interacting cases in the Appendix D.

\section{Example for RESI}

Here we use this formulation to recover the equations for the RESI mechanism of NSDI. The process of RESI goes through each states in our two-electron wavefunction ansatz given by Eq. (30). Hence, it goes through the 'chain'

$$
|0\rangle \underset{\mathbf{d}\left(\mathbf{p}^{\prime \prime}\right)}{\longrightarrow}\left|\mathbf{p}^{\prime \prime}, 0\right\rangle \underset{\mathbf{g}\left(\mathbf{p}^{\prime \prime}, \mathbf{p}, \eta\right)}{\longrightarrow}|\mathbf{p}, \eta\rangle \underset{\mathbf{h}\left(\mathbf{p}, \eta, \mathbf{p}, \mathbf{p}^{\prime}\right)}{\longrightarrow}\left|\mathbf{p}, \mathbf{p}^{\prime}\right\rangle
$$

where the dipole matrix elements underneath are essential for the transitions between states, so must be included to describe RESI. In Fig. 7, the complete Feynman diagram for RESI is shown. In addition to these dipole matrix elements we include those responsible for self propagating the states

$$
\begin{aligned}
& |\mathbf{p}, 0\rangle \rightarrow\left|\mathbf{p}^{\prime}, 0\right\rangle \quad \text { implemented by } \mathbf{g}\left(\mathbf{p}, \mathbf{p}^{\prime}\right), \\
& |\mathbf{p}, \eta\rangle \rightarrow\left|\mathbf{p}^{\prime}, \eta^{\prime}\right\rangle \quad \text { implemented by } \mathbf{h}\left(\mathbf{p}, \eta, \mathbf{p}^{\prime}, \eta^{\prime}\right) \text {, } \\
& \left|\mathbf{p}, \mathbf{p}^{\prime}\right\rangle \rightarrow\left|\mathbf{p}^{\prime \prime}, \mathbf{p}^{\prime \prime \prime}\right\rangle \quad \text { implemented by } \mathbf{i}\left(\mathbf{p}, \mathbf{p}^{\prime}, \mathbf{p}^{\prime \prime}, \mathbf{p}^{\prime \prime \prime}\right) \text {. }
\end{aligned}
$$

The remaining dipole matrix elements will not contribute significantly to RESI and thus we can neglect their contributions to the corresponding part of the time dependent Schrödinger equation. $\mathbf{d}(\mathbf{p}, \eta), \mathbf{d}\left(\mathbf{p}, \mathbf{p}^{\prime}\right)$, and $\mathbf{g}\left(\mathbf{p}, \mathbf{p}^{\prime}, \mathbf{p}^{\prime \prime}\right)$. In this example we will take the simplest case where electron interaction is only considered in the necessary step of the excitation of the second electron. As in the case of SFA in SAE approximation, the crucial point is to determine the most singular parts of the relevant matrix elements and the less singular "rest". SFA will then correspond to the systematic expansion in the "rest".

Below we provide the necessary decompositions of the relevant matrix at two levels: (a) at the level of exact matrix elements, calculated for two-electron Hamiltonian; (b) approximated matrix elements, calculated, neglecting the electron-electron interactions. In this way we will be able to compare with previous SFA results for RESI. We list 
both expressions a) and b) in their most explicit form,

$$
\begin{aligned}
& \mathbf{d}(\mathbf{p})=\mathbf{d}(\mathbf{p}) \simeq \frac{2 e}{\sqrt{2}}\left\langle 0_{1}|\hat{\mathbf{r}}| \mathbf{p}\right\rangle, \\
& \mathbf{g}\left(\mathbf{p}, \mathbf{p}^{\prime}\right)=i e \hbar \nabla_{\mathbf{p}} \delta\left(\mathbf{p}-\mathbf{p}^{\prime}\right)+\hbar \tilde{\mathbf{g}}\left(\mathbf{p}, \mathbf{p}^{\prime}\right) \simeq i e \hbar \nabla_{\mathbf{p}} \delta\left(\mathbf{p}-\mathbf{p}^{\prime}\right)+\hbar \mathbf{g}_{1}\left(\mathbf{p}, \mathbf{p}^{\prime}\right), \\
& \mathbf{h}\left(\mathbf{p}, \eta, \mathbf{p}^{\prime}, \eta^{\prime}\right)=\delta_{\eta \eta^{\prime}}\left(i e \hbar \nabla_{\mathbf{p}} \delta\left(\mathbf{p}-\mathbf{p}^{\prime}\right)+\hbar \tilde{\mathbf{h}}\left(\mathbf{p}, \mathbf{p}^{\prime}, \eta\right)\right)+e \delta\left(\mathbf{p}-\mathbf{p}^{\prime}\right) \tilde{\mathbf{d}}\left(\eta, \eta^{\prime}\right) \\
& \simeq \delta_{\eta \eta^{\prime}}\left(i e \hbar \nabla_{\mathbf{p}} \delta\left(\mathbf{p}-\mathbf{p}^{\prime}\right)+\hbar \mathbf{g}_{1}\left(\mathbf{p}, \mathbf{p}^{\prime}\right)\right)+e \delta\left(\mathbf{p}-\mathbf{p}^{\prime}\right)\left\langle\eta|\hat{\mathbf{r}}| \eta^{\prime}\right\rangle, \\
& \mathbf{h}\left(\mathbf{p}, \eta, \mathbf{p}^{\prime}, \mathbf{p}^{\prime \prime}\right) \simeq e \delta\left(\mathbf{p}-\mathbf{p}^{\prime \prime}\right) \tilde{\mathbf{h}}\left(\mathbf{p}, \mathbf{p}^{\prime}, \eta\right)+e \delta\left(\mathbf{p}-\mathbf{p}^{\prime}\right) \tilde{\mathbf{h}}\left(\mathbf{p}, \mathbf{p}^{\prime \prime}, \eta\right) \\
& \simeq e \delta\left(\mathbf{p}-\mathbf{p}^{\prime \prime}\right)\left\langle\eta|\hat{\mathbf{r}}| \mathbf{p}^{\prime}\right\rangle+e \delta\left(\mathbf{p}-\mathbf{p}^{\prime}\right)\left\langle\eta|\hat{\mathbf{r}}| \mathbf{p}^{\prime \prime}\right\rangle, \\
& \mathbf{i}\left(\mathbf{p}, \mathbf{p}^{\prime}, \mathbf{p}^{\prime \prime}, \mathbf{p}^{\prime \prime \prime}\right) \simeq \delta\left(\mathbf{p}^{\prime}-\mathbf{p}^{\prime \prime \prime}\right)\left(i e \hbar \nabla_{\mathbf{p}} \delta\left(\mathbf{p}-\mathbf{p}^{\prime \prime}\right)+\hbar \tilde{\mathbf{g}}\left(\mathbf{p}, \mathbf{p}^{\prime}, \mathbf{p}^{\prime \prime}, \mathbf{p}^{\prime \prime \prime}\right)\right) \\
& +\delta\left(\mathbf{p}^{\prime}-\mathbf{p}^{\prime \prime}\right)\left(i e \hbar \nabla_{\mathbf{p}} \delta\left(\mathbf{p}-\mathbf{p}^{\prime \prime \prime}\right)+\hbar \tilde{\mathbf{g}}\left(\mathbf{p}, \mathbf{p}^{\prime}, \mathbf{p}^{\prime \prime}, \mathbf{p}^{\prime \prime \prime}\right)\right) \\
& +\delta\left(\mathbf{p}-\mathbf{p}^{\prime \prime \prime}\right)\left(i e \hbar \nabla_{\mathbf{p}^{\prime}} \delta\left(\mathbf{p}^{\prime}-\mathbf{p}^{\prime \prime}\right)+\hbar \tilde{\mathbf{g}}\left(\mathbf{p}, \mathbf{p}^{\prime}, \mathbf{p}^{\prime \prime}, \mathbf{p}^{\prime \prime \prime}\right)\right) \\
& +\delta\left(\mathbf{p}-\mathbf{p}^{\prime \prime}\right)\left(i e \hbar \nabla_{\mathbf{p}^{\prime}} \delta\left(\mathbf{p}^{\prime}-\mathbf{p}^{\prime \prime \prime}\right)+\hbar \tilde{\mathbf{g}}\left(\mathbf{p}, \mathbf{p}^{\prime}, \mathbf{p}^{\prime \prime}, \mathbf{p}^{\prime \prime \prime}\right)\right) \\
& \simeq \delta\left(\mathbf{p}^{\prime}-\mathbf{p}^{\prime \prime \prime}\right)\left(i e \hbar \nabla_{\mathbf{p}} \delta\left(\mathbf{p}-\mathbf{p}^{\prime \prime}\right)+\hbar \mathbf{g}_{1}\left(\mathbf{p}, \mathbf{p}^{\prime}, \mathbf{p}^{\prime \prime}, \mathbf{p}^{\prime \prime \prime}\right)\right) \\
& +\delta\left(\mathbf{p}^{\prime}-\mathbf{p}^{\prime \prime}\right)\left(i e \hbar \nabla_{\mathbf{p}} \delta\left(\mathbf{p}-\mathbf{p}^{\prime \prime \prime}\right)+\hbar \mathbf{g}_{1}\left(\mathbf{p}, \mathbf{p}^{\prime \prime \prime}\right)\right) \\
& +\delta\left(\mathbf{p}-\mathbf{p}^{\prime \prime \prime}\right)\left(i e \hbar \nabla_{\mathbf{p}^{\prime}} \delta\left(\mathbf{p}^{\prime}-\mathbf{p}^{\prime \prime}\right)+\hbar \mathbf{g}_{1}\left(\mathbf{p}^{\prime}, \mathbf{p}^{\prime \prime}\right)\right) \\
& +\delta\left(\mathbf{p}-\mathbf{p}^{\prime \prime}\right)\left(i e \hbar \nabla_{\mathbf{p}^{\prime}} \delta\left(\mathbf{p}^{\prime}-\mathbf{p}^{\prime \prime \prime}\right)+\hbar \mathbf{g}_{1}\left(\mathbf{p}^{\prime}, \mathbf{p}^{\prime \prime \prime}\right)\right)
\end{aligned}
$$

The convention that we use above is that the less singular parts of the matrix elements with tilde are calculated "exactly", taking into account electron-electron interaction, while the matrix with subscript 1 stem from approximate calculation, in which we neglect the electron-electron interactions, so that these matrix elements can be obtained from the corresponding single electron dipole moments, calculated in the SAE approximation. Thus $\hbar \mathbf{g}_{1}\left(\mathbf{p}, \mathbf{p}^{\prime}\right)=\hbar \mathbf{g}\left(\mathbf{p}, \mathbf{p}^{\prime}\right)$ from the previous chapters.

In addition we have perhaps the most important matrix element that describes re-scattering of the electron accompanied by the excitation of the remaining electron,

$$
\mathbf{g}\left(\mathbf{p}, \mathbf{p}^{\prime}, \eta\right)=e \delta\left(\mathbf{p}-\mathbf{p}^{\prime}\right) \mathbf{d}(\mathbf{p}, \eta)+\hbar n \tilde{\mathbf{g}}\left(\mathbf{p}, \mathbf{p}^{\prime}, \eta\right)=e \delta\left(\mathbf{p}-\mathbf{p}^{\prime}\right)\langle 0|\hat{\mathbf{r}}| \eta\rangle_{1}+\hbar \mathbf{g}_{1}\left(\mathbf{p}, \mathbf{p}^{\prime}, \eta\right)
$$

This matrix element we will keep entire, but treat it as a perturbation.

Since we treat $\mathbf{g}\left(\mathbf{p}, \mathbf{p}^{\prime}, \eta\right)$ as a first order perturbation, we can then keep only the most singular parts of the remaining matrix elements, neglecting the less singular parts, $\tilde{\mathbf{g}}\left(\mathbf{p}, \mathbf{p}^{\prime}\right)$, ... etc. These less singular contributions can be very interesting, leading to Coulomb effects such as distortion of interference structures in ATI [24, 243], and should certainly receive some attention. In the present instance we will take the most basic form of RESI neglecting them. Thus, we will take for instance the single electron re-scattering $\tilde{\mathbf{g}}\left(\mathbf{p}, \mathbf{p}^{\prime}\right)=0$ in above equations. Substituting these into the integro-differential equations (see Eq. (E1) in Appendix E) gives a much simplified form:

$$
\begin{aligned}
& \dot{a}(t)=\frac{i}{\hbar} \mathbf{E}(t) \cdot \int d^{3} \mathbf{p} \mathbf{d}(\mathbf{p}) b(\mathbf{p}, t) \\
& \dot{b}(\mathbf{p}, t)=-\frac{i}{\hbar}\left[\left(\frac{\hbar^{2} p^{2}}{2 m}+E_{0}-E_{10}\right) b(\mathbf{p}, t)-\mathbf{E}(t) \mathbf{d}(\mathbf{p}) a(t)-i e \hbar \mathbf{E}(t) \cdot \nabla_{\mathbf{p}} b(\mathbf{p}, t)\right]+\cdots \\
& \dot{c}(\mathbf{p}, \eta, t)=-\frac{i}{\hbar}\left[\left(\frac{\hbar^{2} p^{2}}{2 m}+E_{0}-E_{1 \eta}\right) c(\mathbf{p}, \eta, t)-\mathbf{E}(t) \cdot \int d^{3} \mathbf{p}^{\prime} \mathbf{g}\left(\mathbf{p}^{\prime}, \mathbf{p}, \eta\right) b\left(\mathbf{p}^{\prime}, t\right)-i e \hbar \mathbf{E}(t) \nabla_{\mathbf{p}} c(\mathbf{p}, \eta, t)\right]+\cdots \\
& \dot{d}\left(\mathbf{p}, \mathbf{p}^{\prime}, t\right)=-\frac{i}{\hbar}\left[\left(\frac{\hbar^{2} p^{2}}{2 m}+\frac{\hbar^{2} p^{\prime 2}}{2 m}+E_{0}\right) d\left(\mathbf{p}, \mathbf{p}^{\prime}, t\right)-e \mathbf{E}(t) \cdot \sum_{\eta \neq 0}\left(c\left(\mathbf{p}^{\prime}, \eta, t\right) \tilde{\mathbf{g}}\left(\mathbf{p}, \mathbf{p}^{\prime}, \eta\right)+c(\mathbf{p}, \eta, t) \tilde{\mathbf{g}}\left(\mathbf{p}^{\prime}, \mathbf{p}, \eta\right)\right)\right. \\
&\left.\quad-2 i e \hbar \mathbf{E}(t) \cdot\left(\nabla_{\mathbf{p}}+\nabla_{\mathbf{p}^{\prime}}\right) d\left(\mathbf{p}, \mathbf{p}^{\prime}, t\right)\right]+\cdots
\end{aligned}
$$

The above equations contain only the terms relevant for the perturbative solution in the first order in $\tilde{\mathbf{g}}\left(\mathbf{p}, \mathbf{p}^{\prime}, \eta\right)-$ they have thus reduced to a very simple form. Now integral solutions of each of these equations can be formulated, where $\dot{d}\left(\mathbf{p}, \mathbf{p}^{\prime}, t\right)$ is expressed in terms of $\dot{c}(\mathbf{p}, \eta, t), \dot{c}(\mathbf{p}, \eta, t)$ is in terms of $\dot{b}(\mathbf{p}, t)$ and $\dot{b}(\mathbf{p}, t)$ is in terms of $a(t)$, which we assume to know (or we set to unity for the not-too-strong and not-too-long driving pulses). The solutions are as 
follows:

$$
\begin{aligned}
& b\left(\mathbf{p}^{\prime \prime}, t^{\prime \prime}\right)=\frac{i}{h} \int_{0}^{t^{\prime \prime}} d t^{\prime} \exp \left[-\frac{i}{\hbar} S_{b}\left(\mathbf{p}^{\prime \prime}, t^{\prime}, t^{\prime \prime}\right)\right] \mathbf{d}\left(\mathbf{p}^{\prime \prime}-e \mathbf{A}\left(t^{\prime}\right) / c\right) a\left(t^{\prime}\right) \\
& c\left(\mathbf{p}, \eta, t^{\prime \prime \prime}\right)=\frac{i}{\hbar} \int_{0}^{t^{\prime \prime \prime}} d t^{\prime \prime} \int d^{3} \mathbf{p}^{\prime \prime} \exp \left[-\frac{i}{\hbar} S_{c}\left(\mathbf{p}, t^{\prime \prime}, t^{\prime \prime \prime}\right)\right] \mathbf{E}\left(t^{\prime \prime}\right) \cdot \mathbf{g}\left(\mathbf{p}-e \mathbf{A}\left(t^{\prime \prime}\right) / c, \mathbf{p}^{\prime \prime}-e \mathbf{A}\left(t^{\prime \prime}\right) / c, \eta\right) b\left(\mathbf{p}^{\prime \prime}, t^{\prime \prime}\right), \quad(54) \\
& d\left(\mathbf{p}, \mathbf{p}^{\prime}, t\right)= \frac{i}{\hbar} \int_{0}^{t} d t^{\prime \prime \prime} \exp \left[\frac{i}{\hbar} S_{d}\left(\mathbf{p}, \mathbf{p}^{\prime}, t^{\prime \prime \prime}, t\right)\right] \mathbf{E}\left(t^{\prime \prime \prime}\right) \cdot \sum_{\eta \neq 0}\left(\tilde{\mathbf{g}}\left(\mathbf{p}-e \mathbf{A}\left(t^{\prime \prime \prime}\right) / c, \mathbf{p}^{\prime}-e \mathbf{A}\left(t^{\prime \prime \prime}\right) / c, \eta\right) c\left(\mathbf{p}^{\prime}, \eta, t^{\prime \prime \prime}\right) \quad(55)\right. \\
&\left.+\tilde{\mathbf{g}}\left(\mathbf{p}^{\prime}-e \mathbf{A}\left(t^{\prime \prime \prime}\right) / c, \mathbf{p}-e \mathbf{A}\left(t^{\prime \prime \prime}\right) / c, \eta\right) c\left(\mathbf{p}, \eta, t^{\prime \prime \prime}\right)\right)
\end{aligned}
$$

where

$$
\begin{aligned}
S_{b}\left(\mathbf{p}^{\prime \prime}, t^{\prime}, t^{\prime \prime}\right) & =\int_{t^{\prime}}^{t^{\prime \prime}} d \tau\left[\frac{1}{2 m}\left(\mathbf{p}^{\prime \prime}-e \mathbf{A}(\tau) / c\right)^{2}+E_{0}-E_{10}\right] \\
S_{c}\left(\mathbf{p}, t^{\prime \prime}, t^{\prime \prime \prime}\right) & =\int_{t^{\prime \prime}}^{t^{\prime \prime \prime}} d \tau\left[\frac{1}{2 m}(\mathbf{p}-e \mathbf{A}(\tau) / c)^{2}+E_{0}-E_{1 \eta}\right] \\
S_{d}\left(\mathbf{p}, \mathbf{p}^{\prime}, t^{\prime \prime \prime}, t\right) & =\int_{t^{\prime \prime \prime}}^{t} d \tau\left[\frac{1}{2 m}(\mathbf{p}-e \mathbf{A}(\tau) / c)^{2}+\frac{1}{2 m}\left(\mathbf{p}^{\prime}-e \mathbf{A}(\tau) / c\right)^{2}\right]
\end{aligned}
$$

The S-Matrix transition amplitude of this process can be related to the above expression in the following way

$$
\begin{aligned}
M\left(\mathbf{p}, \mathbf{p}^{\prime}\right) & =\lim _{t \rightarrow \infty}\left\langle\mathbf{p}, \mathbf{p}^{\prime} \mid \psi(t)\right\rangle \\
& =\lim _{t \rightarrow \infty} d\left(\mathbf{p}, \mathbf{p}^{\prime}, t\right)
\end{aligned}
$$

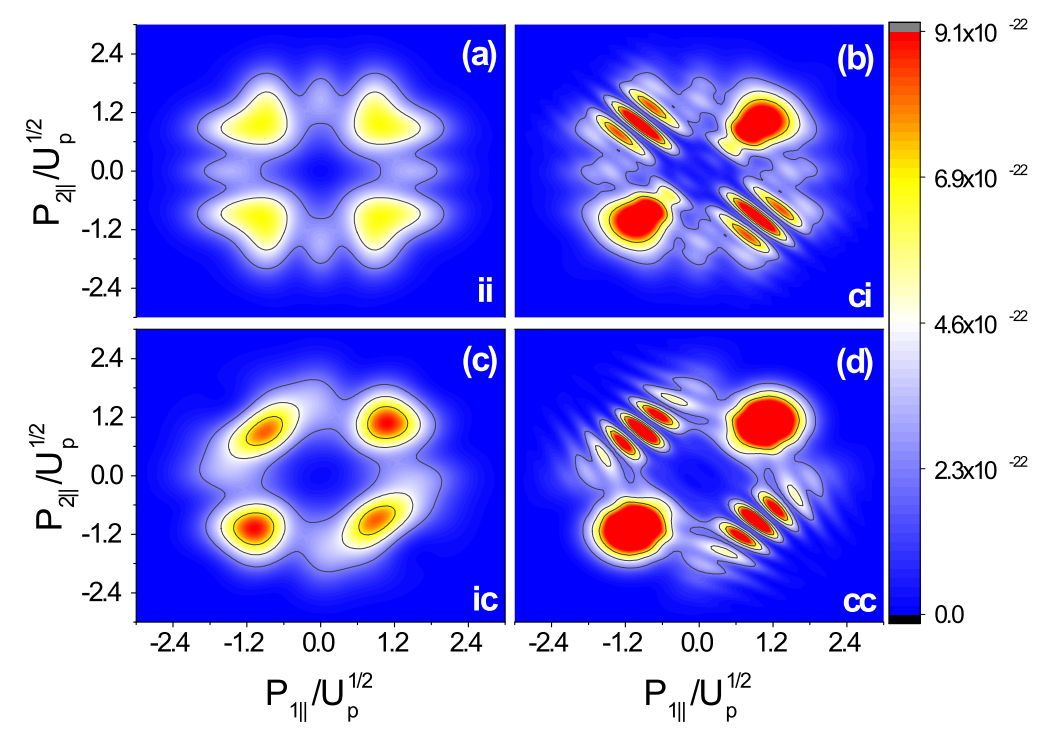

FIG. 8. Momentum distribution of RESI for argon showing coherent and incoherent sums of pathways relating to different intermediate excited states and pathways related by symmetries. Whether the sum is coherent or incoherent (denoted c and i) is given in the bottom right for both the pathways relating to symmetries and excited states respectively. The ponderomotive energy is given by $U_{\mathrm{p}}=0.1$ a.u. $\left(I=4.56 \times 10^{13} \mathrm{~W} / \mathrm{cm}^{2}\right)$ corresponding to an angular frequency $\omega=0.057$ a.u. or wavelength $\lambda=800 \mathrm{~nm}$. 
We get thus the final result for the RESI amplitude

$$
\begin{aligned}
d\left(\mathbf{p}, \mathbf{p}^{\prime}, t\right)= & \left(\frac{i}{\hbar}\right)^{3} \sum_{\eta \neq 0} \int_{0}^{t} d t^{\prime \prime \prime} \int_{0}^{t^{\prime \prime \prime}} d t^{\prime \prime} \int_{0}^{t^{\prime \prime}} d t^{\prime} \int d^{3} \mathbf{p}^{\prime \prime} \exp \left[\frac{i}{\hbar} S_{d}\left(\mathbf{p}, \mathbf{p}^{\prime}, t^{\prime \prime \prime}, t\right)\right] \\
\times & \mathbf{E}\left(t^{\prime \prime \prime}\right) \cdot\left(\tilde{\mathbf{g}}\left(\mathbf{p}^{\prime}-e \mathbf{A}\left(t^{\prime \prime \prime}\right) / c, \mathbf{p}-e \mathbf{A}\left(t^{\prime \prime \prime}\right) / c, \eta\right) \exp \left[-\frac{i}{\hbar} S_{c}\left(\mathbf{p}, t^{\prime \prime}, t^{\prime \prime \prime}\right)\right]\right. \\
& \times \mathbf{E}\left(t^{\prime \prime}\right) \cdot \mathbf{g}\left(\mathbf{p}-e \mathbf{A}\left(t^{\prime \prime}\right) / c, \mathbf{p}^{\prime \prime}-e \mathbf{A}\left(t^{\prime \prime}\right) / c, \eta\right) \exp \left[-\frac{i}{\hbar} S_{b}\left(\mathbf{p}^{\prime \prime}, t^{\prime}, t^{\prime \prime}\right)\right] \mathbf{d}\left(\mathbf{p}^{\prime \prime}-e \mathbf{A}\left(t^{\prime}\right) / c\right) a\left(t^{\prime}\right) \\
+ & \left.\left\{\mathbf{p}^{\prime} \rightarrow \mathbf{p}\right\}\right)
\end{aligned}
$$

In this expression the three actions will be combined to give the SFA action for the RESI processes. The three integrals with the times $t^{\prime}, t^{\prime \prime}$ and $t^{\prime \prime \prime}$ can be associated with first ionization, recollision excitation and final ionization respectively. The integral over momentum can be related to the intermediate momentum. These integrals can be solved by the saddle point approximation, which makes this problem computation tractable, as was done in [179, 244]. Therein probability distributions for monochromatic fields were calculated for the momentum components parallel to the laser field polarisation, where the components perpendicular to the laser field polarisation are integrated over, shown in Fig. 8. This shows that different pathways for the the RESI process will interfere, by plotting coherent and incoherent sums. These are pathways related to ionization via different excited states; for argon there are six pathways that contribute, as well as pathways related to those via symmetries such as the indistinguishability of electrons.

\section{Example for EII}

Using the same logic it is easy to calculate do the same for the EII of NSDI. Using the same restrictions as before only the integro-differential equation for $d\left(\mathbf{p}, \mathbf{p}^{\prime}, t\right)$ needs to be changed. This can easily be seen in the EII "chain" (see Fig. 9)

$$
|0\rangle \underset{\mathbf{d}\left(\mathbf{p}^{\prime \prime}\right)}{\longrightarrow}\left|\mathbf{p}^{\prime \prime}, 0\right\rangle \underset{\mathbf{g}\left(\mathbf{p}^{\prime \prime}, \mathbf{p}, \mathbf{p}^{\prime}\right)}{\longrightarrow}\left|\mathbf{p}, \mathbf{p}^{\prime}\right\rangle
$$

Here, we neglect the dipole matrix element for recollision excitation RESI contribution given by $\mathbf{g}\left(\mathbf{p}, \mathbf{p}^{\prime}, \eta\right)$, and instead include in a similar way the matrix element $\mathbf{g}\left(\mathbf{p}, \mathbf{p}^{\prime}, \mathbf{p}^{\prime \prime}\right)$. We can proceed as before, and now the integro-differential equation for $d\left(\mathbf{p}, \mathbf{p}^{\prime}, t\right)$ can be written out, this time it only depends on $b\left(\mathbf{p}^{\prime \prime}, t^{\prime \prime}\right)$ and is given by,

$$
\begin{gathered}
\dot{d}\left(\mathbf{p}, \mathbf{p}^{\prime}, t\right)=-\frac{i}{\hbar}\left[\left(\frac{\hbar^{2} p^{2}}{2 m}+\frac{\hbar^{2} p^{\prime 2}}{2 m}+E_{0}\right) d\left(\mathbf{p}, \mathbf{p}^{\prime}, t\right)-\mathbf{E}(t) \cdot \int d^{3} \mathbf{p}^{\prime \prime} \mathbf{g}\left(\mathbf{p}, \mathbf{p}^{\prime}, \mathbf{p}^{\prime \prime}\right) b\left(\mathbf{p}^{\prime \prime}, t^{\prime \prime}\right)\right. \\
\left.-2 \mathbf{E}(t) \cdot\left(\nabla_{\mathbf{p}}+\nabla_{\mathbf{p}^{\prime}}\right) d\left(\mathbf{p}, \mathbf{p}^{\prime}, t\right)\right]
\end{gathered}
$$

Then the solution can be written as

$$
\begin{aligned}
d\left(\mathbf{p}, \mathbf{p}^{\prime}, t\right)= & \frac{i}{\hbar} \int_{0}^{t} d t^{\prime \prime} \int_{0}^{t^{\prime \prime}} d t^{\prime} \int d^{3} \mathbf{p}^{\prime \prime} \exp \left[\frac{i}{\hbar} S_{d}\left(\mathbf{p}, \mathbf{p}^{\prime}, t^{\prime \prime}, t\right)\right] \mathbf{E}\left(t^{\prime \prime}\right) \mathbf{g}\left(\mathbf{p}-e \mathbf{A}\left(t^{\prime \prime}\right) / c, \mathbf{p}^{\prime}-e \mathbf{A}\left(t^{\prime \prime}\right) / c, \mathbf{p}^{\prime \prime}\right) \\
& \times \exp \left[-\frac{i}{\hbar} S_{b}\left(\mathbf{p}^{\prime \prime}, t^{\prime}, t^{\prime \prime}\right)\right] \mathbf{d}\left(\mathbf{p}^{\prime \prime}-e \mathbf{A}\left(t^{\prime}\right) / c\right) a\left(t^{\prime}\right)
\end{aligned}
$$

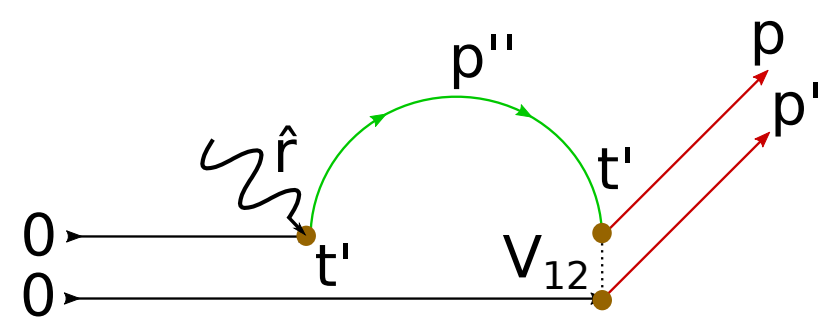

FIG. 9. The complete EI process showing the two transitions. The two nodes marked at $t^{\prime}$ and $t^{\prime \prime}$ show the two transitions identified in the chain in Eq. (62). 
Ultimately, both the EII and RESI mechanism can be included in $d\left(\mathbf{p}, \mathbf{p}^{\prime}, t\right)$ and it will still be integrable and, as expected, will simply be equal to the sum of these two solutions.

\section{LARGE MOLECULES AND TARGETS IN STRONG LASER FIELDS}

In this section we formulate the general problem we want to attack with the help of SFA. The Hamiltonian describing a multi-atomic molecule or atomic cluster has the following general form:

$$
\hat{H}=\hat{H}_{\mathrm{nuc}}+\hat{H}_{\mathrm{el}},
$$

where the nuclear hamiltonian reads

$$
\hat{H}_{\mathrm{nuc}}=\sum_{i=1}^{N} \frac{\mathbf{P}_{i}^{2}}{2 M_{i}}+V\left(\mathbf{R}_{1}, \ldots, \mathbf{R}_{N}\right)
$$

with the inter-nuclear potential

$$
V\left(\mathbf{R}_{1}, \ldots, \mathbf{R}_{N}\right)=\frac{1}{2} \sum_{i \neq j}^{N} \frac{Z_{i} Z_{j} e^{2}}{\left|\mathbf{R}_{i}-\mathbf{R}_{j}\right|}
$$

where $N$ is the number of nuclei involved, $i$ enumerates the nuclei, and $\mathbf{R}_{i}, \mathbf{P}_{i}$ are their positions and momenta, respectively, $Z_{i}$ are the nuclear charges, and $M_{i}$ the nuclear masses. In principle, we could include more complex nucleus-nucleus interactions, taking into account deeply-bound electrons via effective potentials and similar methods. We neglect here the influence of the laser electric field on the nucleus - they are simply too heavy to be affected by the short laser pulses.

The electronic Hamiltonian depends parametrically on the positions of the nuclei, via

$$
\hat{H}_{\mathrm{el}}=\sum_{i=1}^{M} \frac{\mathbf{p}_{i}^{2}}{2 m}+\frac{1}{2} \sum_{i \neq j}^{M} \frac{e^{2}}{\left|\mathbf{r}_{i}-\mathbf{r}_{j}\right|}-\sum_{i, j}^{M, N} \frac{Z_{j} e^{2}}{\left|\mathbf{r}_{i}-\mathbf{R}_{j}\right|}-\sum_{i=1}^{M} e \mathbf{E}(t) \mathbf{r}_{i}
$$

Here $M$ is the number of electrons involved, $i$ enumerates them, and $\mathbf{r}_{i}, \mathbf{p}_{i}$ are their positions and momenta, respectively. Again, we could replace bare Coulomb potentials by the dressed effective ones. Also, we assume that the target is large, but still smaller than the wavelength, so that a global dipole approximation holds.

\section{Born-Oppenheimer Approximation}

In the following we assume that the nuclear motion is slower than that of the electrons, so we use the BornOppenheimer approach. To this is aim we first determine the electronic wave function, $\Psi_{\mathrm{el}}\left(\left\{\mathbf{r}_{i}\right\}_{i=1}^{M}, t ;\left\{\mathbf{R}_{i}\right\}_{i=1}^{N}\right)$, in Dirac's notation denoted as $\left|\Psi_{\mathrm{el}}(t)\right\rangle$ that fulfils TDSE with fixed nuclear positions

$$
i \hbar \frac{d}{d t}\left|\Psi_{\mathrm{el}}(t)\right\rangle=\hat{H}_{\mathrm{el}}\left|\Psi_{\mathrm{el}}(t)\right\rangle
$$

We define then instantaneous electronic potential for the nuclei

$$
E_{\mathrm{el}}\left(\left\{\mathbf{R}_{i}\right\}_{i=1}^{N}, t\right)=\left\langle\Psi_{\mathrm{el}}(t)\left|\hat{H}_{\mathrm{el}}\right| \Psi_{\mathrm{el}}(t)\right\rangle,
$$

and treat the motion of the nuclei classically and solve the resulting Newton equations

$$
\begin{aligned}
\frac{d \mathbf{R}_{i}}{d t} & =\frac{\mathbf{P}_{i}}{M_{i}}, \\
\frac{d \mathbf{P}_{i}}{d t} & =-\nabla_{\mathbf{R}_{i}} V\left(\mathbf{R}_{1}, \ldots, \mathbf{R}_{N}\right)-\nabla_{\mathbf{R}_{i}} E_{\mathrm{el}}\left(\mathbf{R}_{1}, \ldots, \mathbf{R}_{N}\right) .
\end{aligned}
$$

The solutions of these classical equations are then inserted into the electronic TDSE, and so on. In general, it has to be done self-consistently. We will discuss below, couple of cases when some simplifications are possible. 


\section{Single Active Electron Approximation}

The theory formulated above can be reduced to a single-electron TDSE using the SAE approximation. In principle, it can be done in a same way as it is done in the static case for atoms or molecules. The only difference is that we now have to consider the fact that the effective potential must now be time-dependent through the parametric dependence on the nuclear coordinates,

$$
\hat{H}_{\mathrm{eff}}=\frac{\mathbf{p}^{2}}{2 m}+V_{\mathrm{eff}}\left(\mathbf{R}_{1}(t), \ldots, \mathbf{R}_{N}(t), t\right)-e \mathbf{E}(t) \mathbf{r}
$$

While calculations of $V_{\text {eff }}$ for atoms belongs still to the domain of the atomic physics, calculations of the effective potential for molecules and, especially in the dynamical situations clearly require use of molecular physics and quantum theoretical chemistry methods.

\section{A. Strong Field Approximation for quenched molecules}

The equations of the above section are very complex. There are some situations, however, when they can be radically simplified. One example of such a situation is the instant quench, in which the molecule is suddenly stripped of, say, one of the electrons, or photoexcited to a certain well-defined state. This can be achieved, for instance, applying an ultrashort attosecond XUV or soft X-ray pulse to the molecule. Right after the pulse, the molecule will find itself in the ground state corresponding to one missing electron, or in the well-defined excited state. In both situations, the nuclei configuration will be by no means stable. The molecule will start to vibrate, rotate, and maybe even dissociate.

If the excitation occurs to a weakly bound molecular state, the following vibrations or dissociation will occur on a rather slow time scale of $100 \mathrm{fs}$ to $1 \mathrm{ps}$. In that case, the HHG or ATI caused by an intense few-femtosecond pulse may be used for an instant imaging of the dynamically changing molecular structure (for seminal experiments see Refs. 245, 246). If the electron removal or excitation occurs to a strongly-bound state, the resulting dynamics might be much faster: stripping of electrons, for instance, might lead to dissociation completely controlled by the Coulomb forces, and occurring on the timescales of an atomic unit (fractions of a femtosecond). These are the situations we want to consider in this section.

\section{SFA and molecular dynamics}

If we then apply a short femtosecond laser pulse in the mid-infrared range, we may expect that similarly as in the standard HHG or ATI processes, the femtosecond laser induced electronic dynamics will not affect the intrinsic molecular dynamics. That means that, from the point of view of nuclei, we can replace the electronic Hamiltonian

$$
\hat{H}_{\mathrm{eff}}=\frac{\mathbf{p}^{2}}{2 m}+V_{\mathrm{eff}}\left(\mathbf{R}_{1}(t), \ldots, \mathbf{R}_{N}(t), t\right)-e \mathbf{E}(t) \mathbf{r}=\hat{H}_{0}-e \mathbf{E}(t) \mathbf{r},
$$

with

$$
\hat{H}_{0}=\frac{\mathbf{p}^{2}}{2 m}+V_{\mathrm{eff}}\left(\mathbf{R}_{1}(t), \ldots, \mathbf{R}_{N}(t), t\right)
$$

The Born-Oppenheimer Newton equations for the nuclei can then be solved self-consistently, as

$$
\begin{aligned}
\frac{d \mathbf{R}_{i}}{d t} & =\frac{\mathbf{P}_{i}}{M_{i}}, \\
\frac{d \mathbf{P}_{i}}{d t} & =-\nabla_{\mathbf{R}_{i}} V\left(\mathbf{R}_{1}, \ldots, \mathbf{R}_{N}\right)-\nabla_{\mathbf{R}_{i}} E_{\mathrm{el}}\left(\mathbf{R}_{1}, \ldots, \mathbf{R}_{N}\right),
\end{aligned}
$$

where

$$
E_{\mathrm{el}}\left(\left\{\mathbf{R}_{i}\right\}_{i=1}^{N}, t\right)=\left\langle\Psi_{\mathrm{el}}(t)\left|\hat{H}_{0}\left(\left\{\mathbf{R}_{i}\right\}_{i=1}^{N}, t\right)\right| \Psi_{\mathrm{el}}(t)\right\rangle
$$

Assuming the that the ionization during the process is weak, the contribution of the continuum part of the electronic wavefunction will give negligible contribution to the electronic energy, so that

$$
E_{\mathrm{el}}\left(\left\{\mathbf{R}_{i}\right\}_{i=1}^{N}, t\right) \simeq\left\langle\Psi_{0}(t)\left|\hat{H}_{0}\left(\left\{\mathbf{R}_{i}\right\}_{i=1}^{N}, t\right)\right| \Psi_{0}(t)\right\rangle|a(t)|^{2}
$$


where $\left|\Psi_{0}(t)\right\rangle$ is the time-dependent ground electronic state, and $a(t)$ is the probability amplitude of being in this state. If we know $|a(t)|^{2}$ (for instance, if we can assume that $|a(t)|^{2} \simeq 1$ ), then the solutions can be simply introduced into Eq. (90), and one can then calculate explicitly - i.e. without self-consistency conditions-both $\left|\Psi_{0}(t)\right\rangle$ and the corresponding continuum functions that fulfill

$$
\begin{aligned}
E_{0}\left(\left\{\mathbf{R}_{i}\right\}_{i=1}^{N}, t\right)\left|\Psi_{0}(t)\right\rangle & =\hat{H}_{0}\left(\left\{\mathbf{R}_{i}\right\}_{i=1}^{N}, t\right)\left|\Psi_{0}(t)\right\rangle \\
\frac{1}{2 m} \mathbf{p}^{2}|\mathbf{p}(t)\rangle & =\hat{H}_{0}\left(\left\{\mathbf{R}_{i}\right\}_{i=1}^{N}, t\right)|\mathbf{p}(t)\rangle .
\end{aligned}
$$

Both of these functions depend explicitly on time through the time dependence of the positions of the nuclei. Note, that the equations can be even more simplified if we can simplify the effects of $E_{0}$ in Eq. (76) âAS the equations will not even require self-consistency! For instance, in the case of stripping of, say, $K$ electrons, for large internuclei distance, the only effect of $E_{0}$ in Eq. (76) will be to screen the nuclei charges, that is replace $Z_{i}$ s by $\tilde{Z}_{i}$ s, where $\sum_{i} Z_{i}=\sum_{i} \tilde{Z}_{i}-K$

\section{SFA for a quenched molecule}

The expression derived above implicitly assumes that we proceed in fact as in Section III. That is, we write the full electronic wave function as

$$
|\Psi(t)\rangle=e^{i I_{p}(t) t / \hbar+i \phi_{B}(t)}\left(a(t)\left|\Psi_{0}(t)\right\rangle+\int d^{3} \mathbf{p} b(\mathbf{p}, t)|\mathbf{p}(t)\rangle\right) .
$$

where we set $I_{p}(t)=-E_{0}(t)$. The new effect here is $\phi_{B}(t)=\left\langle\Psi_{0}(t) \mid \partial_{t} \Psi_{0}(t)\right\rangle$ - the Berry phase arising from projecting/expanding the electronic wave function in the time dependent basis. The equations still have practically the same form as before; for instance the direct transition amplitude fulfills:

$$
\partial_{t} b_{0}(\mathbf{p}, t)=-\frac{i}{\hbar}\left(\frac{\mathbf{p}^{2}}{2 m}+\tilde{I}_{p}(t)\right) b_{0}(\mathbf{p}, t)+\frac{i}{\hbar} \mathbf{E}(t) \cdot \mathbf{d}(\mathbf{p}, t) a(t)+\mathbf{E}(t) \cdot \nabla_{\mathbf{v}} b_{0}(\mathbf{p}, t),
$$

where we have included now the Berry phase in $\tilde{I}_{p}(t)=I_{p}(t)+\hbar \phi_{B}(t)$. Note that the Berry phase is nonzero if and only if the ground-state wavefunction is complex. This typically happens if the time-reversal symmetry is broken, i.e. for instance in the presence of a magnetic field or a so-called "artificial" gauge field. Also, the matrix element now depends explicitly on time, through the time dependence of the positions of the nuclei.

\section{Generalized SFA expression for a quenched molecule}

The above equations for the electronic dynamics together with the Newton equations (75), (76), together with the expressions for the electronic energy (77), (78), (79), allow us to derive thus:

- The direct ATI amplitude:

$$
\begin{array}{rl}
b_{0}(\mathbf{p}, t)=\frac{i}{\hbar} \int_{0}^{t} & d t^{\prime} \mathbf{E}\left(t^{\prime}\right) \cdot \mathbf{d}\left(\mathbf{p}-e \mathbf{A}\left(t^{\prime}\right) / c, t\right) a\left(t^{\prime}\right) \\
& \quad \times \exp \left(-\frac{i}{\hbar} \int_{t^{\prime}}^{t} d \tilde{t}\left[\frac{1}{2 m}(\mathbf{p}-e \mathbf{A}(\tilde{t}) / c)^{2}+\tilde{I}_{p}(t)\right]\right) .
\end{array}
$$

- The re-scattering amplitude:

$$
\begin{aligned}
b_{1}(\mathbf{p}, t)= & -\int_{0}^{t} d t^{\prime} \exp \left[-i S\left(\mathbf{p}, t, t^{\prime}\right) / \hbar\right] \mathbf{E}\left(t^{\prime}\right) \cdot \int_{0}^{t^{\prime}} d t^{\prime \prime} \int d^{3} \mathbf{p}^{\prime} \mathbf{g}\left(\mathbf{p}-e \mathbf{A}\left(t^{\prime}\right) / c, \mathbf{p}^{\prime}-e \mathbf{A}\left(t^{\prime}\right) / c, t\right) \\
& \times \mathbf{E}\left(t^{\prime \prime}\right) \cdot \mathbf{d}\left(\mathbf{p}^{\prime}-e \mathbf{A}\left(t^{\prime \prime}\right) / c, t\right) a\left(t^{\prime \prime}\right) \exp \left[-i S\left(\mathbf{p}^{\prime}, t^{\prime}, t^{\prime \prime}\right) / \hbar\right]
\end{aligned}
$$

where

$$
S\left(\mathbf{p}, t, t^{\prime}\right)=\int_{t^{\prime}}^{t} d \tilde{t}\left[\frac{1}{2 m}(\mathbf{p}-e \mathbf{A}(\tilde{t}) / c)^{2}+\tilde{I}_{p}(\tilde{t})\right]
$$


- The time-dependent dipole moment

$$
\begin{aligned}
\langle\mathbf{x}(t)\rangle=\operatorname{Re}\left[\frac{i}{\hbar}\right. & \int_{0}^{t} d t^{\prime} \int d^{3} \mathbf{p} a^{*}(t) \mathbf{d}(\mathbf{p}-e \mathbf{A}(t) / c) \mathbf{E}\left(t^{\prime}\right) \cdot \mathbf{d}\left(\mathbf{p}-e \mathbf{A}\left(t^{\prime}\right) / c\right) a\left(t^{\prime}\right) \\
& \left.\times \exp \left(-i \int_{t^{\prime}}^{t} d \tilde{t}\left[\frac{1}{2 m}(\mathbf{p}-e \mathbf{A}(\tilde{t}) / c)^{2}+I_{p}\right] / \hbar\right)\right]
\end{aligned}
$$

Note that if $|a(t)|^{2}$ is "known", then the solutions do not require self-consistency. Otherwise, they have to be obtained in the manner discussed below.

\section{B. SFA for large targets}

Here we consider another situation: the molecule (a large target) is initially in the ground state, and is impinged by an intense, short (few laser cycles) laser pulse in the mid-infrared range. This pulse causes the ionization of the single active electron, and induces thus structural dynamics of the target, i.e. the motion of the nuclei. Amazingly, the expressions describing the quantities of interest are exactly the same as in the previous section. The way to obtain them, however, is much more complex: now we have to determine the evolution of $\mathbf{R}_{i}(t)$ and $\mathbf{P}_{i}(t)$ simultaneously and self-consitently with the dynamics of the electronic wave function, $\left|\Psi_{\mathrm{el}}(t)\right\rangle$.

The protocol to follow is thus:

1. Calculate the electronic state (the ground state of $\hat{H}_{\text {eff }}$ ),

$$
\left.E_{\mathrm{el}}\left(\left\{\mathbf{R}_{i}(0)\right\}_{i=1}^{N}, t\right) \Psi_{\mathrm{el}}(0)\right\rangle=\hat{H}_{\mathrm{eff}}\left(\left\{\mathbf{R}_{i}(0)\right\}_{i=1}^{N}, 0\right)\left|\Psi_{\mathrm{el}}(0)\right\rangle
$$

for the initial positions of the nuclei $\pm \Delta \mathbf{R}_{i}$ (to be able to calculate gradients).

2. Propagate the equations for nuclei,

$$
\begin{aligned}
\frac{d \mathbf{R}_{i}}{d t} & =\frac{\mathbf{P}_{i}}{M_{i}} \\
\frac{d \mathbf{P}_{i}}{d t} & =-\nabla_{\mathbf{R}_{i}} V\left(\mathbf{R}_{1}, \ldots, \mathbf{R}_{N}\right)-\nabla_{\mathbf{R}_{i}} E_{\mathrm{el}}\left(\mathbf{R}_{1}, \ldots, \mathbf{R}_{N}\right)
\end{aligned}
$$

to the next time instant, $t$. Calculate the new $\left\{\mathbf{R}_{i}(t)\right\}_{i=1}^{N}$.

3. Calculate the new electronic state $\left|\Psi_{\mathrm{el}}(t)\right\rangle$. This is the state propagated using $\hat{H}_{\text {eff }}$,

$$
i \hbar \frac{d}{d t}\left|\Psi_{\mathrm{el}}(t)\right\rangle=\hat{H}_{\mathrm{el}}\left(\left\{\mathbf{R}_{i}(t)\right\}_{i=1}^{N}, t\right)\left|\Psi_{\mathrm{el}}(t)\right\rangle
$$

for the actual positions of the nuclei $\pm \Delta \mathbf{R}_{i}$ (to be able to calculate gradients). Note that this propagation should be done using the SFA ansatz (80)). Calculate then

$$
E_{\mathrm{el}}\left(\left\{\mathbf{R}_{i}(t)\right\}_{i=1}^{N}, t\right)=\left\langle\Psi_{\mathrm{el}}(t) \hat{H}_{\mathrm{eff}}\left(\left\{\mathbf{R}_{i}(t)\right\}_{i=1}^{N}, 0\right) \mid \Psi_{\mathrm{el}}(t)\right\rangle
$$

for the actual positions of the nuclei $\pm \Delta \mathbf{R}_{i}$ (to be able to calculate gradients).

4. Calculate

$$
E_{\mathrm{el}}\left(\left\{\mathbf{R}_{i}(t)\right\}_{i=1}^{N}, t\right)=\left\langle\Psi_{\mathrm{el}}(t)\left|\hat{H}_{\mathrm{el}}\left(\left\{\mathbf{R}_{i}(t)\right\}_{i=1}^{N}, 0\right)\right| \Psi_{\mathrm{el}}(t)\right\rangle
$$

\section{Go to 2.}

Obviously, the above procedure is quite complex, but it does not present giant numerical challenges, and it is relatively straightforward to implement. Evidently, it is much easier than solving the TDSE involving classical (BornOppenheimer) dynamics of the nuclei, the feasibility of which is not entirely obvious. 


\section{SFA and quantum molecular dynamics}

The use of classical Newton equations for molecules in dissociation or vibration processes might be questionable. There is a simple method of including certain aspects of the quantum motion of molecules that we describe now. Our starting assumption is the generalization of the SFA ansatz to the full wave function:

$$
|\Psi(t)\rangle=\xi_{0}\left(\mathbf{R}_{1}, \ldots, \mathbf{R}_{N}\right) a(t)\left|\Psi_{0}\right\rangle+\xi_{1}\left(\mathbf{R}_{1}, \ldots, \mathbf{R}_{N}\right) \int d^{3} \mathbf{p} b(\mathbf{p}, t)|\mathbf{p}\rangle
$$

where $\xi_{0}, \xi_{1}$ are the normalized wave functions of the nuclei for the molecule with $M, M-1$ electrons correspondingly. As before $\hat{H}_{\text {eff }}=\hat{H}_{0}-e \mathbf{E}(t) \mathbf{r}$, whereas

$$
\hat{H}_{0}=\frac{\mathbf{p}^{2}}{2 m}+V_{\mathrm{eff}}\left(\mathbf{R}_{1}, \ldots, \mathbf{R}_{N}(t)\right) .
$$

The electronic ground state is now time-independent, but it does explicitly depend on the nuclear positions via

$$
E_{0}\left(\mathbf{R}_{1}, \ldots, \mathbf{R}_{N}\right)\left|\Psi_{0}\right\rangle=\hat{H}_{0}\left|\Psi_{0}\right\rangle
$$

Similarly, the states in the continuum do not depend on time, but on the nuclear positions, entering via $\hat{H}_{0}$ as

$$
\frac{\mathbf{p}^{2}}{2 m}|\mathbf{p}\rangle=\hat{H}_{0}|\mathbf{p}\rangle
$$

We still use the Born-Oppenheimer approximation, but in the quantum version. Also, we use different Hamiltonians for the non-ionized and ionized part of the molecular electronic dynamics. Thus, for $\xi_{0}\left(\mathbf{R}_{1}, \ldots, \mathbf{R}_{N}\right)$ we use

$$
\hat{H}_{\text {nuc }, 0}=\sum_{i=1}^{N} \frac{\mathbf{P}_{i}^{2}}{2 M_{i}}+V\left(\mathbf{R}_{1}, \ldots, \mathbf{R}_{N}\right)+|a(t)|^{2}\left\langle\Psi_{0}(t)\left|\hat{H}_{0}\right| \Psi_{0}(t)\right\rangle
$$

while for $\xi_{1}\left(\mathbf{R}_{1}, \ldots, \mathbf{R}_{N}\right)$ we use simply

$$
\hat{H}_{\mathrm{nuc}, 1}=\sum_{i=1}^{N} \frac{\mathbf{P}_{i}^{2}}{2 M_{i}}+V\left(\mathbf{R}_{1}, \ldots, \mathbf{R}_{N}\right)
$$

We neglect here the laser part of the electronic energy, as well as the kinetic energy of electrons in the continuum. Note that the equation (98) can be solved without any self-consistency conditions. As in the previous sections, equation (97) can also be solved that way, provided that the time dependence of $|a(t)|^{2}$ is known.

The last point is the derivation of the SFA equation. To this end we assume that the quantum fluctuations of the nuclear positions are small, and replace the $\mathbf{R}_{i}$ dependence in $\left|\Psi_{0}(t)\right\rangle$ by the average $\overline{\mathbf{R}}_{i}(t)=\int d^{3} \mathbf{R} \mathbf{R}_{i}\left|\xi_{0}(\mathbf{R}, t)\right|^{2}$. Similarly, we replace the $\mathbf{R}_{i}$ dependence in the continuum part by the average $\overline{\mathbf{R}}_{i}(t)=\int d^{3} \mathbf{R} \mathbf{R},\left|\xi_{1}(\mathbf{R}, t)\right|^{2} ;$ after that trick, the SFA equations can be projected on the normalized functions $\xi_{0,1}(t)$. This leads to the following modified equations:

$$
\partial_{t} b_{0}(\mathbf{p}, t)=-\frac{i}{\hbar}\left(\frac{\mathbf{p}^{2}}{2 m}+I_{p}(t)\right) b_{0}(\mathbf{p}, t)+\mathbf{E}(t) \cdot \nabla_{\mathbf{v}} b_{0}(\mathbf{p}, t)+\frac{i}{\hbar} \mathbf{E}(t) \cdot \mathbf{d}(\mathbf{p}, t)\left\langle\xi_{0}(t) \mid \xi_{1}(t)\right\rangle a(t)
$$

and

$$
\partial_{t} a(t)=\frac{i}{\hbar}\left\langle\xi_{1}(t) \mid \xi_{0}(t)\right\rangle \int d^{3} \mathbf{p} \mathbf{E}(t) \cdot \mathbf{d}(\mathbf{p}, t) b_{0}(\mathbf{p}, t)
$$

As we see, the final equation depends only on the overlap $\left\langle\xi_{1}(t) \mid \xi_{0}(t)\right\rangle$, generally called the nuclear autocorrelation function, which, despite the fact that the positions of the nuclei in each branch of the process are quite "classical", might become very small as the positions of the nuclei in the two channels change. This can then seriously limit the HHG and LIED signals from the process, both for the direct and re-scattering parts [247-249]. 


\section{SFA FOR SOLIDS}

In addition to the generation of harmonics in gases, the past decade has seen a broad interest in the generation of harmonics from condensed matter, where the higher density of emitters offers the possibility of much brighter emission, both in liquids [250, 251] and in solids, starting with the seminal experiment in a bulk crystal [181]. While the disorder of the liquid phase makes spectroscopic studies of its structure more complicated, the rigid structure of solids allows for a much deeper understanding of the emission mechanisms as well as, potentially, broad and detailed high-harmonic spectroscopy studies.

This is because the continuum which is explored by the electron released in a solid by a strong-field excitation from the valence to the conduction band contains much more structure than the quadratic band of a free electron, as shown in Fig. 10, and the dispersion induced by this structured continuum produces non-harmonic motion which leads to the emission of so-called "intraband" emission. Moreover, in atoms, the ionized electron leaves behind a stationary hole which is bound to the parent ion and cannot be displaced in space, but in solids this is no longer the case, and the motion of the hole in the valence band also needs to be considered; nevertheless, when the hole and electron meet, they can recombine and emit so-called interband harmonics, exactly as in the atomic case. However, despite that similarity, there are important differences, since that recombination can happen away from the origin, the electron and hole trajectories are subject to more complicated dynamics in their dispersive bands, and the bands themselves contain nontrivial parallel-transport effects that produce, through a nonzero geometrical phase, additional 'anomalous' velocity terms that also contribute to the harmonic emission.

To a good approximation, the electronic dynamics in a solid driven by a strong low-frequency laser pulse is governed by the semiconductor Bloch equations (SBE), as derived in Ref. 253,

$$
\begin{aligned}
\dot{n}_{m}(\mathbf{K}, t)= & \frac{i}{\hbar} s_{m} e \mathbf{E}(t) \cdot \mathbf{d}_{c v}^{*}(\mathbf{K}-e \mathbf{A}(t) / c) \pi(\mathbf{K}, t)+\text { c.c. }, \\
\dot{\pi}(\mathbf{K}, t)= & -\frac{i}{\hbar}\left[\varepsilon_{g}(\mathbf{K}-e \mathbf{A}(t) / c)+e \mathbf{E}(t) \cdot \boldsymbol{\xi}_{g}(\mathbf{K}-e \mathbf{A}(t) / c)-i \frac{\hbar}{T_{2}}\right] \pi(\mathbf{K}, t) \\
& -\frac{i}{\hbar} e \mathbf{E}(t) \cdot \mathbf{d}_{c v}(\mathbf{K}-e \mathbf{A}(t) / c) w(\mathbf{K}, t) .
\end{aligned}
$$

where $n_{m}$ is the population in band $m, \pi$ is the inter-band coherence (where we assume for simplicity a two-band model $), w=n_{c}-n_{v}$ is the population difference between the valence and conduction bands, $s_{c}=-s_{v}=1, \mathbf{d}_{m m}(\mathbf{k})$ is the inter-band dipole moment, and $\boldsymbol{\xi}_{g}(\mathbf{k})=\boldsymbol{\xi}_{c}(\mathbf{k})-\boldsymbol{\xi}_{v}(\mathbf{k})$ is the difference in the Berry connections of the two bands [253, supplemental material]. These variables give rise to the harmonic emission via the intra and inter-band

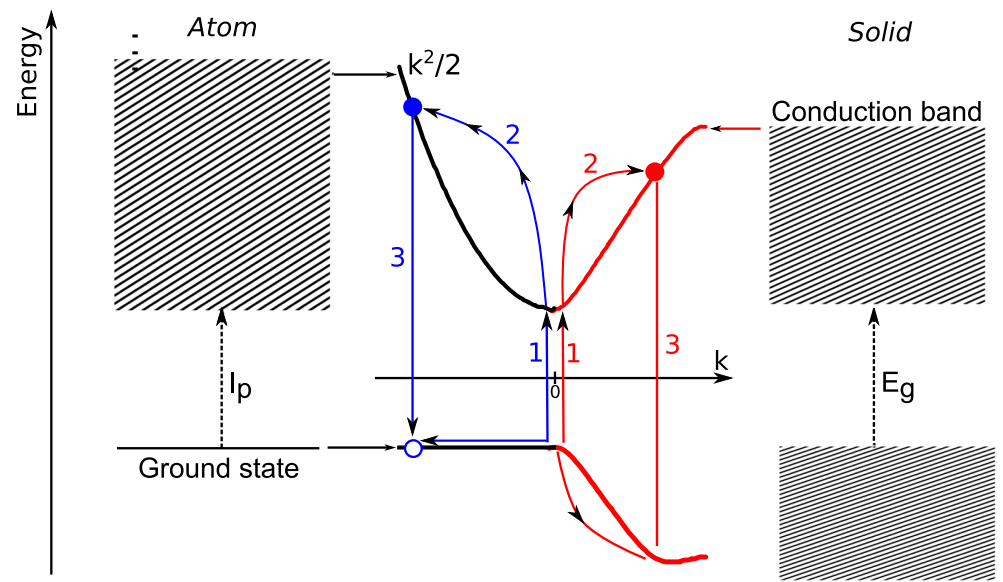

FIG. 10. Sketch of the differences between the harmonic-emission processes in atoms and solids. In atoms, the continuum band is parabolic, so the electron's motion in the continuum does not emit harmonics, and the hole it leaves behind remains stationary in a flat band. In a solid, on the other hand, the hole can also move, and both holes and electrons experience dispersive forces in their continuum motion, which leads to the emission of intraband harmonics. However, when electrons and holes meet, they can also emit interband harmonics analogous to the gas-phase harmonics. Adapted from Ref. 252. 
(b)

(a)

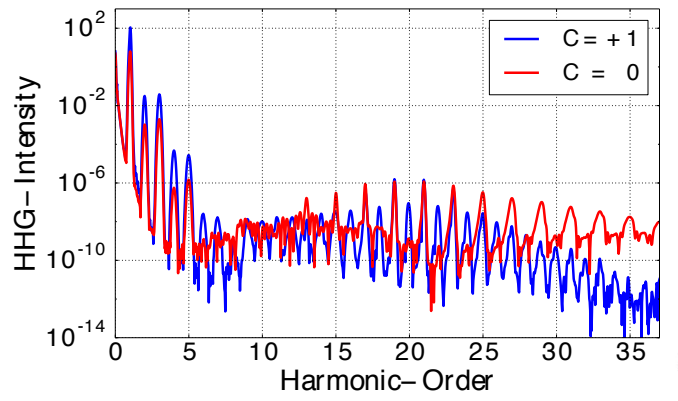

\section{.}
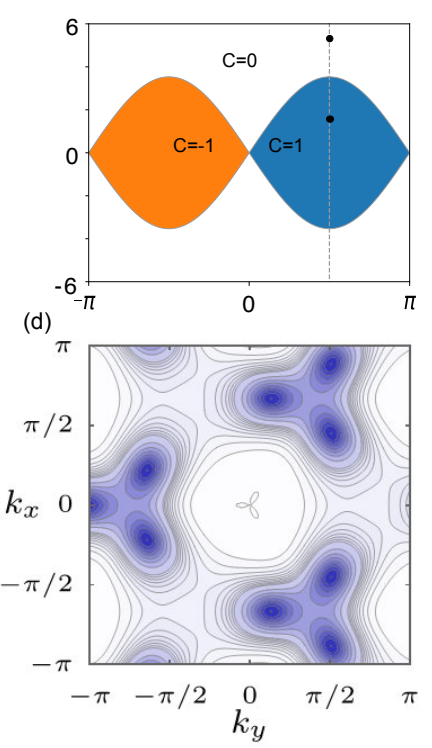

(c)

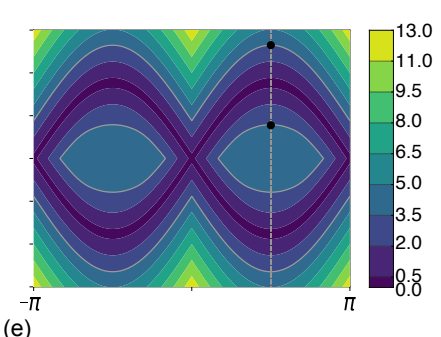

(e)

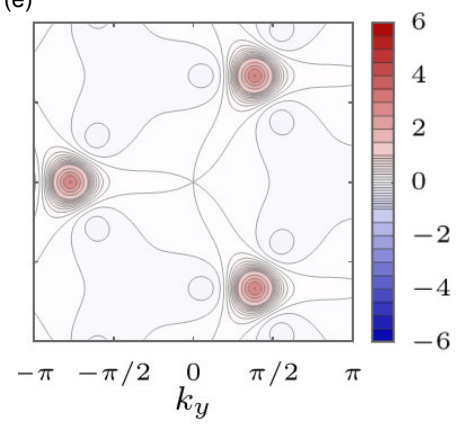

FIG. 11. Traces in HHG of a topological phase transition in a two-dimensional material, calculated from the semiconductor Bloch equations $(101,102)$ on the Haldane model in Ref. 253. (a) High-harmonic spectrum for two closely-related sets of parameters, with equivalent bandgaps but on either side of the topological phase transition characterized with different Chern numbers $C(\mathrm{~b})$, showing a distinct suppression of even harmonics on the (trivial/nontrivial) side, despite the fact that the symmetries of the Hamiltonian allow those harmonics and the bandgaps are essentially identical (c); it is only when one considers the Berry curvature for those materials $(\mathrm{d}, \mathrm{e})$ that the differences in the spectrum can be explained.

components of the total current,

$$
\begin{aligned}
\mathbf{J}_{\mathrm{ra}}(t) & =e \sum_{m} \int_{\overline{\mathrm{BZ}}} d^{3} \mathbf{K} \mathbf{v}_{m}(\mathbf{K}-e \mathbf{A}(t) / c) n_{m}(\mathbf{K}, t), \\
\mathbf{J}_{\mathrm{er}}(t) & =e \frac{d}{d t} \int_{\overline{\mathrm{BZ}}} d^{3} \mathbf{K} \mathbf{d}_{c v}^{*}(\mathbf{K}-e \mathbf{A}(t) / c) \pi(\mathbf{K}, t)+\text { c.c. }
\end{aligned}
$$

The semiconductor Bloch equations $(101,102)$ are too complex to solve directly other than numerically but, as in the atomic case, they are subject to the Keldysh approximation [33] which allows us to give an approximate expression for the current,

$$
\begin{aligned}
J_{\mathrm{er}}^{(i)}(t)=-\mathrm{i} \sum_{j} & \frac{d}{d t} \int_{t_{0}}^{t} d t^{\prime} \int_{\overline{\mathrm{BZ}}} d^{3} \mathbf{K}\left|d_{c v}^{(i)}(\mathbf{K}-e \mathbf{A}(t) / c)\right|\left|d_{c v}^{(j)}\left(\mathbf{K}-e \mathbf{A}\left(t^{\prime}\right) / c\right)\right| E^{(j)}\left(t^{\prime}\right) \\
& \times e^{-\mathrm{i} S\left(\mathbf{K}, t, t^{\prime}\right) / \hbar-\left(t-t^{\prime}\right) / T_{2}+i\left(\phi_{c v}^{(j)}(\mathbf{K}, t)-\phi_{c v}^{(i)}(\mathbf{K}, t)\right)}+\text { c.c. },
\end{aligned}
$$

where $S\left(\mathbf{K}, t, t^{\prime}\right)$ is the so called quasi-classical action for the electron-hole and is defined according to:

$$
S\left(\mathbf{K}, t, t^{\prime}\right)=\int_{t^{\prime}}^{t}\left[\varepsilon_{g}\left(\mathbf{K}-e \mathbf{A}\left(t^{\prime \prime}\right) / c\right)+e \mathbf{E}\left(t^{\prime \prime}\right) \cdot \boldsymbol{\xi}_{g}\left(\mathbf{K}-e \mathbf{A}\left(t^{\prime \prime}\right) / c\right)-\hbar \frac{d}{d t^{\prime \prime}} \phi_{c v}^{(j)}\left(\mathbf{K}-e \mathbf{A}\left(t^{\prime \prime}\right) / c\right)\right] d t^{\prime \prime} .
$$

This forms the heart of the SFA description of high-harmonic emission in a crystalline solid, and it contains all of the semiclassical dynamics for the electron trajectories in the conduction band as well as the hole's trajectory in the valence band [182, 183, 252].

Traditional treatments of HHG in solids have worked in one-dimensional configurations where the geometrical-phase element of (106) can be set to zero using an appropriate choice of gauge for the Bloch-function basis, in which case the kinematics of the electron and hole wavepackets are fully determined by the band structure [182, 254, 255]. However, there are materials where this gauge transformation is not possible, due to the existence of a Berry curvature on one or more of the bands, and the geometric-phase terms in (106) cannot be neglected. This Berry curvature is crucial for a wide array of solid-state effects [256-258], and it is often the driving ingredient of nontrivial phenomena. Its 
presence in the SFA harmonic-emission current (105) means that it can in principle be measured via HHG observables, and indeed recent experimental $[186,187]$ and theoretical $[253,259]$ works show that this is the case; we showcase in Fig. 11 some of our recent results on that front.

\section{ACKNOWLEDGMENTS}

We thank Sougato Bose, Paul Corkum, Anne L'Huillier, Yann Mairesse and Pascal Salières for enlightening discussions. This work was supported by National Science Centre Poland-Symfonia Grant No. 2016/20/W/ST4/00314. We acknowledge also the project Advanced Research Using High Intensity Laser Produced Photons and Particles (No. CZ. 02.1.01/0.0/0.0/16_019/0000789) from the European Regional Development Fund (ADONIS), Ministerio de Economía y Competitividad through Plan Nacional (Grant No. FIS2016-79508-P FISICATEAMO, and Severo Ochoa Excellence Grant No. SEV-2015-0522), funding from the European Union Horizon 2020 Research and Innovation Programme under the Marie Sklodowska-Curie Grant Agreement No. 641272 Laserlab-Europe (Grant No. EU-H2020 654148), Fundació Privada Cellex, and Generalitat de Catalunya (Grant No. SGR 1341 and CERCA Programme). N.S. was supported by the Erasmus Mundus Doctorate Program Europhotonics (Grant No. 159224-1-2009-1-FRERAMUNDUS-EMJD). N.S., A.C., E.P., and M.L. acknowledge ERC AdG OSYRIS, EU FETPRO QUIC. K.A. and J.B. additionally acknowledge ERC AdG Transformer (788218), Ministerio de Economía y Competitividad through Plan Nacional FIS2017-89536-P and Agencia de Gestió d'Ajuts Universitaris i de Recerca (AGAUR) with 2017 SGR 1639. E.P. acknowledges Cellex-ICFO-MPQ Fellowship funding. A.D. is financed by a "Juan de la Cierva" fellowship (IJCI-2017-33180). J.A.P.-H. acknowledges Spanish Ministerio de Economía, Industria y Competitividad (MINECO) through the PALMA Grant No. FIS2016-81056-R, from LaserLab Europe IV Grant No. 654148, and from the Regional Government, Junta de Castilla y León, Grant No. CLP087U16.

[1] D. Strickland and G. Mourou. Compression of amplified chirped optical pulses. Opt. Commun. 55 no. 6, pp. 447-449 (1985).

[2] Groundbreaking inventions in laser physics: optical tweezers and generation of high-intensity, ultra-short optical pulses. Scientific background on the Nobel Prize in Physics 2018. The Nobel Prize Committee in Physics (2018).

[3] M. Nisoli, S. De Silvestri, O. Svelto et al. Compression of high-energy laser pulses below 5 fs. Opt. Lett. 22 no. 8 , pp. 522-524 (1997). Author eprint.

[4] M. Hemmer, M. Baudisch, A. Thai, A. Couairon and J. Biegert. Self-compression to sub-3-cycle duration of mid-infrared optical pulses in dielectrics. Opt. Express 21 no. 23, p. 28095 (2013). arXiv:1304.5955.

[5] M. F. Ciappina, J. A. Pérez-Hernández, A. Landsman et al. Attosecond physics at the nanoscale. Rep. Prog. Phys. 80, p. 054401 (2017). arXiv:1607.01480.

[6] F. Krausz and M. Y. Ivanov. Attosecond physics. Rev. Mod. Phys. 81 no. 1, pp. 163-234 (2009).

[7] M. Drescher, M. Hentschel, R. Kienberger et al. X-ray pulses approaching the attosecond frontier. Science 291 no. 5510 , pp. 1923-1927 (2001).

[8] K. Zhao, Q. Zhang, M. Chini et al. Tailoring a 67 attosecond pulse through advantageous phase-mismatch. Opt. Lett. 37 no. 18, pp. 3891-3893 (2012).

[9] F. Silva, S. M. Teichmann, S. L. Cousin, M. Hemmer and J. Biegert. Spatio-temporal isolation of attosecond soft x-ray pulses in the water window. Nature Commun. 6, p. 6611 (2015).

[10] P. Salières, A. L'Huillier, P. Antoine and M. Lewenstein. Study of spatial and temporal coherence of high order harmonics. In B. Bederson and H. Walther (eds.), Adv. At. Mol. Opt. Phys., vol. 41, pp. 83-142 (Academic Press, 1999). arXiv:quant$\mathrm{ph} / 9710060$.

[11] M. Lewenstein and A. L'Huillier. Principles of Single Atom Physics: High-Order Harmonic Generation, Above-Threshold Ionization and Non-Sequential Ionization. In T. Brabec (ed.), Strong Field Laser Physics, pp. $147-183$ (Springer, New York, 2009).

[12] G. G. Paulus, F. Grasbon, H. Walther et al. Absolute-phase phenomena in photoionization with few-cycle laser pulses. Nature 414, p. 182 (2001). Author eprint.

[13] J. Itatani, J. Levesque, D. Zeidler et al. Tomographic imaging of molecular orbitals. Nature 432, p. 867 (2004).

[14] C. I. Blaga, J. Xu, A. D. DiChiara et al. Imaging ultrafast molecular dynamics with laser-induced electron diffraction. Nature 483, p. 194 (2012). OSU eprint.

[15] M. G. Pullen, B. Wolter, A. T. Le et al. Imaging an aligned polyatomic molecule with laser-induced electron diffraction. Nature Commun. 6, p. 7262 (2015).

[16] A. Baltuška, T. Udem, M. Uiberacker et al. Attosecond control of electronic processes by intense light fields. Nature 421, p. 611 (2003).

[17] B. Wolter, C. Lemell, M. Baudisch et al. Formation of very-low-energy states crossing the ionization threshold of argon atoms in strong mid-infrared fields. Phys. Rev. A 90 no. 6, p. 063424 (2014). arXiv:1410.5629. 
[18] I. J. Sola, E. Mével, L. Elouga et al. Controlling attosecond electron dynamics by phase-stabilized polarization gating. Nature Phys. 2, p. 319 (2006).

[19] D. Milošević, G. Paulus, D. Bauer and W. Becker. Above-threshold ionization by few-cycle pulses. J. Phys. B: At. Mol. Opt. Phys. 39 no. 14, p. R203 (2006).

[20] T. Zuo, A. D. Bandrauk and P. B. Corkum. Laser-induced electron diffraction: a new tool for probing ultrafast molecular dynamics. Chem. Phys. Lett 259 no. 3-4, pp. 313-320 (1996). HAL eprint.

[21] M. Lein, J. P. Marangos and P. L. Knight. Electron diffraction in above-threshold ionization of molecules. Phys. Rev. A 66 no. 5, p. 051404 (2002). ICL eprint.

[22] C. Lin, A.-T. Le, Z. Chen, T. Morishita and R. Lucchese. Strong-field rescattering physics-self-imaging of a molecule by its own electrons. J. Phys. B: At. Mol. Opt. Phys. 43 no. 12, p. 122001 (2010). KSU eprint.

[23] J. Xu, C. I. Blaga, K. Zhang et al. Diffraction using laser-driven broadband electron wave packets. Nature Commun. 5, p. 4635 (2014).

[24] M. Meckel, D. Comtois, D. Zeidler et al. Laser-induced electron tunneling and diffraction. Science 320 no. 5882 , pp. 1478-1482 (2008). Stanford e-print.

[25] T. Morishita, A.-T. Le, Z. Chen and C. D. Lin. Accurate retrieval of structural information from laser-induced photoelectron and high-order harmonic spectra by few-cycle laser pulses. Phys. Rev. Lett. 100 no. 1, p. 013903 (2008). KSU eprint.

[26] J. Xu, C. I. Blaga, A. D. DiChiara et al. Laser-induced electron diffraction for probing rare gas atoms. Phys. Rev. Lett. 109 no. 23, p. 233002 (2012). KSU eprint.

[27] F. H. Faisal. Multiple absorption of laser photons by atoms. J. Phys. B: At. Mol. Phys. 6 no. 4, p. L89 (1973).

[28] H. R. Reiss. Effect of an intense electromagnetic field on a weakly bound system. Phys. Rev. A 22 no. 5, pp. 1786-1813 (1980).

[29] M. Lewenstein, K. C. Kulander, K. J. Schafer and P. H. Bucksbaum. Rings in above-threshold ionization: a quasiclassical analysis. Phys. Rev. A 51 no. 2, pp. 1495-1507 (1995).

[30] M. V. Frolov, D. V. Knyazeva, N. L. Manakov et al. Analytic model for the description of above-threshold ionization by an intense short laser pulse. Phys. Rev. A 89 no. 6, p. 063419 (2014). UNL eprint.

[31] W. Becker, F. Grasbon, R. Kopold et al. Above-threshold ionization: From classical features to quantum effects. Adv. At. Mol. Opt. Phys. 48, pp. 35-98 (2002). Author eprint.

[32] O. I. Tolstikhin, T. Morishita and S. Watanabe. Adiabatic theory of ionization of atoms by intense laser pulses: onedimensional zero-range-potential model. Phys. Rev. A 81 no. 3, p. 033415 (2010).

[33] L. Keldysh. Ionization in the field of a strong electromagnetic wave. Sov. Phys. JETP 20 no. 5 , p. 1307 (1965). [Zh. Eksp. Teor. Fiz. 47 no. 5, p. 1945 (1965)].

[34] P. Agostini, F. Fabre, G. Mainfray, G. Petite and N. K. Rahman. Free-free transitions following six-photon ionization of xenon atoms. Phys. Rev. Lett. 42 no. 17, pp. 1127-1130 (1979).

[35] P. Kruit, J. Kimman and M. J. V. der Wiel. Absorption of additional photons in the multiphoton ionisation continuum of xenon at 1064, 532 and 440 nm. J. Phys. B: At. Mol. Phys. 14 no. 19, p. L597 (1981).

[36] F. Fabre, G. Petite, P. Agostini and M. Clement. Multiphoton above-threshold ionisation of xenon at 0.53 and $1.06 \mu \mathrm{m}$. J. Phys. B: At. Mol. Phys. 15 no. 9, p. 1353 (1982).

[37] P. Kruit, J. Kimman, H. G. Muller and M. J. van der Wiel. Electron spectra from multiphoton ionization of xenon at 1064, 532, and 355 nm. Phys. Rev. A 28 no. 1, pp. 248-255 (1983).

[38] A. L'Huillier and P. Balcou. High-order harmonic generation in rare gases with a 1-ps 1053-nm laser. Phys. Rev. Lett. 70 no. 6, pp. $774-777$ (1993).

[39] A. McPherson, G. Gibson, H. Jara et al. Studies of multiphoton production of vacuum-ultraviolet radiation in the rare gases. J. Opt. Soc. Am. B 4 no. 4, pp. 595-601 (1987).

[40] A. l'Huillier, L. A. Lompre, G. Mainfray and C. Manus. Multiply charged ions induced by multiphoton absorption in rare gases at $0.53 \mu \mathrm{m}$. Phys. Rev. A 27 no. 5, pp. 2503-2512 (1983).

[41] A. L'Huillier, L. A. Lompre, G. Mainfray and C. Manus. Multiply charged ions induced by multiphoton absorption processes in rare-gas atoms at $1.064 \mu \mathrm{m}$. J. Phys. B: At. Mol. Phys. 16 no. 8, p. 1363 (1983).

[42] G. Mainfray and C. Manus. Multiphoton ionization of atoms. Rep. Prog. Phys. 54 no. 10, pp. 1333-1372 (1991).

[43] M. Uiberacker, T. Uphues, M. Schultze et al. Attosecond real-time observation of electron tunnelling in atoms. Nature 446 no. 7136, pp. 627-32 (2007).

[44] M. Ammosov, N. Delone and V. Krainov. Tunnel ionization of complex atoms and of atomic ions in an alternating electromagnetic field. Sov. Phys. JETP 64 no. 6, p. 1191 (1986). [Zh. Eksp. Teor. Fiz. 91 no. 6, p. 2008 (1986)].

[45] H. Brandi, L. Davidovich and N. Zagury. Non-perturbative methods applied to multiphoton ionization. J. Phys. Colloques 43 no. C2, pp. 397-405 (1982). HAL eprint.

[46] H. S. A. Neto, L. Davidovich and P. W. Milonni. Comment on "multiphoton-ionization transition amplitudes and the Keldysh approximation". Phys. Rev. A 41 no. 7, pp. 4095-4095 (1990).

[47] W. Becker, L. Davidovich and J. K. McIver. Keldysh-like expansion for above-threshold ionization. Phys. Rev. A 49 no. 2, pp. 1131-1140 (1994).

[48] Z. Białynicka-Birula. Exactly soluble model of continuum-continuum transitions in strong laser beams. Phys. Rev. A 28 no. 2, pp. 836-844 (1983).

[49] Z. Deng and J. H. Eberly. Effect of coherent continuum-continuum relaxation and saturation in multiphoton ionization. Phys. Rev. Lett. 53 no. 19, pp. 1810-1813 (1984).

[50] K. Rzążewski and R. Grobe. Saturation of continuum-continuum transitions in multiphoton absorption. Phys. Rev. Lett. 
54 no. 15, pp. 1729-1729 (1985).

[51] J. Eberly, J. Javanainen and K. Rzążewski. Above-threshold ionization. Phys. Rep. 204 no. 5, pp. 331-383 (1991).

[52] N. M. Kroll and K. M. Watson. Charged-particle scattering in the presence of a strong electromagnetic wave. Phys. Rev. A 8 no. 2, pp. 804-809 (1973).

[53] N. M. Kroll and K. M. Watson. Inelastic atom-atom scattering within an intense laser beam. Phys. Rev. A 13 no. 3 , pp. 1018-1033 (1976).

[54] M. Lewenstein, J. Mostowski and M. Trippenbach. Multichannel decay and continuum-continuum transitions in abovethreshold ionisation. J. Phys. B: At. Mol. Phys. 18 no. 14, p. L461 (1985).

[55] J. Grochmalicki, J. Kukliński and M. Lewenstein. Above-threshold ionisation and electron scattering in intense laser fields. J. Phys. B: At. Mol. Phys. 19 no. 22, p. 3649 (1986).

[56] M. Lewenstein. Collective effects at high laser intensities and multiple ionisation. J. Phys. B: At. Mol. Phys. 19 no. 9 , p. L309 (1986).

[57] J. Javanainen, J. H. Eberly and Q. Su. Numerical simulations of multiphoton ionization and above-threshold electron spectra. Phys. Rev. A 38 no. 7, pp. 3430-3446 (1988).

[58] J. H. Eberly, Q. Su and J. Javanainen. Nonlinear light scattering accompanying multiphoton ionization. Phys. Rev. Lett. 62 no. 8, pp. 881-884 (1989).

[59] J. H. Eberly, Q. Su and J. Javanainen. High-order harmonic production in multiphoton ionization. J. Opt. Soc. Am. B 6 no. 7, pp. 1289-1298 (1989).

[60] J. H. Eberly, Q. Su and J. Javanainen. Nonlinear light scattering accompanying multiphoton ionization. Phys. Rev. Lett. 62 no. 8, pp. 881-884 (1989).

[61] Q. Su and J. H. Eberly. Model atom for multiphoton physics. Phys. Rev. A 44 no. 9, pp. 5997-6008 (1991).

[62] R. Grobe and J. H. Eberly. Photoelectron spectra for a two-electron system in a strong laser field. Phys. Rev. Lett. 68 no. 19, pp. 2905-2908 (1992).

[63] R. Grobe and J. H. Eberly. One-dimensional model of a negative ion and its interaction with laser fields. Phys. Rev. A 48 no. 6, pp. 4664-4681 (1993).

[64] D. Dundas, K. T. Taylor, J. S. Parker and E. S. Smyth. Double-ionization dynamics of laser-driven helium. J. Phys. B: At. Mol. Opt. Phys. 32 no. 9, p. L231 (1999).

[65] K. Taylor, J. Parker, K. Meharg and D. Dundas. Laser-driven helium at 780 nm. Eur. Phys. J. D 26 no. 1, pp. 67-71 (2003).

[66] J. S. Parker, L. R. Moore, D. Dundas and K. T. Taylor. Double ionization of helium at 390 nm. J. Phys. B: At. Mol. Opt. Phys. 33 no. 20, p. L691.

[67] A. Emmanouilidou, J. S. Parker, L. R. Moore and K. T. Taylor. Direct versus delayed pathways in strong-field nonsequential double ionization. New J. Phys. 13 no. 4, p. 043001.

[68] J. Feist, S. Nagele, R. Pazourek et al. Nonsequential two-photon double ionization of helium. Phys. Rev. A 77 no. 4 , p. 043420 (2008). arXiv:0803.0511.

[69] R. Pazourek, J. Feist, S. Nagele and J. Burgdörfer. Attosecond streaking of correlated two-electron transitions in helium. Phys. Rev. Lett. 108 no. 16, p. 163001 (2012). arXiv:1112.3273.

[70] K. Sacha and B. Eckhardt. Pathways to double ionization of atoms in strong fields. Phys. Rev. A 63 no. 4 , p. 043414 (2001).

[71] B. Eckhardt and K. Sacha. Classical threshold behaviour in a $(1+1)$-dimensional model for double ionization in strong fields. J. Phys. B: At. Mol. Opt. Phys. 39 no. 18, p. 3865 (2006). arXiv:physics/0605195.

[72] J. S. Prauzner-Bechcicki, K. Sacha, B. Eckhardt and J. Zakrzewski. Time-resolved quantum dynamics of double ionization in strong laser fields. Phys. Rev. Lett. 98 no. 20, p. 203002 (2007).

[73] J. S. Prauzner-Bechcicki, K. Sacha, B. Eckhardt and J. Zakrzewski. Quantum model for double ionization of atoms in strong laser fields. Phys. Rev. A 78 no. 1, p. 013419 (2008).

[74] J. H. Thiede, B. Eckhardt, D. K. Efimov, J. S. Prauzner-Bechcicki and J. Zakrzewski. Ab initio study of time-dependent dynamics in strong-field triple ionization. Phys. Rev. A 98 no. 3, p. 031401 (2018).

[75] C. Ruiz, L. Plaja, L. Roso and A. Becker. Ab initio calculation of the double ionization of helium in a few-cycle laser pulse beyond the one-dimensional approximation. Phys. Rev. Lett. 96 no. 5, p. 053001 (2006).

[76] A. Staudte, C. Ruiz, M. Schöffler et al. Binary and recoil collisions in strong field double ionization of helium. Phys. Rev. Lett. 99 no. 26, p. 263002 (2007).

[77] D. K. Efimov, A. Maksymov, J. S. Prauzner-Bechcicki et al. Restricted-space ab initio models for double ionization by strong laser pulses. Phys. Rev. A 98 no. 1, p. 013405 (2018). arXiv:1803.08364.

[78] K. J. Schafer, B. Yang, L. F. DiMauro and K. C. Kulander. Above threshold ionization beyond the high harmonic cutoff. Phys. Rev. Lett. 70 no. 11, pp. 1599-1602 (1993).

[79] D. N. Fittinghoff, P. R. Bolton, B. Chang and K. C. Kulander. Observation of nonsequential double ionization of helium with optical tunneling. Phys. Rev. Lett. 69 no. 18, pp. 2642-2645 (1992).

[80] B. Walker, B. Sheehy, L. F. DiMauro et al. Precision measurement of strong field double ionization of helium. Phys. Rev. Lett. 73 no. 9, pp. 1227-1230 (1994).

[81] A. L'Huillier, K. J. Schafer and K. C. Kulander. Theoretical aspects of intense field harmonic generation. J. Phys. B: At. Mol. Opt. Phys. 24 no. 15, p. 3315 (1991).

[82] J. L. Krause, K. J. Schafer and K. C. Kulander. High-order harmonic generation from atoms and ions in the high intensity regime. Phys. Rev. Lett. 68 no. 24, pp. 3535-3538 (1992).

[83] J. G. Leopold and I. C. Percival. Microwave ionization and excitation of Rydberg atoms. Phys. Rev. Lett. 41 no. 14, pp. 
944-947 (1978).

[84] I. C. Percival. Semiclassical theory of Bound States. In I. Prigogine and S. Rice (eds.), Adv. Chem. Phys., vol. 36, pp. 1-61 (Wiley, New York, 1977).

[85] G. Casati, B. V. Chirikov, D. L. Shepelyansky and I. Guarneri. Relevance of classical chaos in quantum mechanics: the hydrogen atom in a monochromatic field. Phys. Rep. 154 no. 2, pp. 77-123 (1987). Author eprint.

[86] J. Grochmalicki, M. Lewenstein and K. Rzążewski. Stabilization of atoms in superintense laser fields: Is it real?. Phys. Rev. Lett. 66 no. 8, pp. 1038-1041 (1991).

[87] M. Gajda, J. Grochmalicki, M. Lewenstein and K. Rzążewski. Stabilization of atoms in ultrastrong laser fields: a classical approach. Phys. Rev. A 46 no. 3, pp. 1638-1653 (1992).

[88] K. Rzążewski, M. Lewenstein and P. Salières. Multielectron stabilization of atoms in a laser field: classical perspective. Phys. Rev. A 49 no. 2, pp. 1196-1201 (1994).

[89] M. Wójcik, J. Zakrzewski and K. Rzążewski. Classical aspects of quantum localization in microwave ionization of H atoms. Phys. Rev. A 52 no. 4, pp. R2523-R2526 (1995).

[90] P. J. Ho and J. Eberly. Argon-like three-electron trajectories in intense-field double and triple ionization. Opt. Express 15 no. 4, pp. 1845-1850 (2007).

[91] J. Chen, J. Liu, L. B. Fu and W. M. Zheng. Interpretation of momentum distribution of recoil ions from laser-induced nonsequential double ionization by semiclassical rescattering model. Phys. Rev. A 63 no. 1, p. 011404 (2000).

[92] N. I. Shvetsov-Shilovski, M. Lein, L. B. Madsen et al. Semiclassical two-step model for strong-field ionization. Phys. Rev. A 94 no. 1, p. 013415 (2016). arXiv:1604.05123.

[93] P. J. Ho, R. Panfili, S. L. Haan and J. H. Eberly. Nonsequential double ionization as a completely classical photoelectric effect. Phys. Rev. Lett. 94 no. 9, p. 093002 (2005).

[94] F. Mauger, C. Chandre and T. Uzer. Strong field double ionization: the phase space perspective. Phys. Rev. Lett. 102 no. 17 , p. 173002 (2009).

[95] R. Panfili, J. H. Eberly and S. L. Haan. Comparing classical and quantum dynamics of strong-field double ionization. Opt. Express 8 no. 7, pp. 431-435 (2001).

[96] S. A. Berman, J. Dubois, C. Chandre, M. Perin and T. Uzer. Coherent buildup of high-order harmonic radiation: the classical perspective. Phys. Rev. A 97 no. 6, p. 061402 (2018). arXiv:1801.09727.

[97] M. Ivanov and O. Smirnova. Multielectron high harmonic generation: simple man on a complex plane. In T. Schultz and M. Vrakking (eds.), Attosecond and XUV Physics: Ultrafast Dynamics and Spectroscopy, pp. 201-256 (Wiley-VCH, Weinheim, 2014). arXiv:1304.2413.

[98] M. Y. Kuchiev. Atomic antenna. JETP Lett. 45 no. 7, pp. 404-406 (1987). [Pis'ma Zh. Eksp. Teor. Fiz. 45 no. 7 , pp. 319-321 (1987)].

[99] F. Brunel. Not-so-resonant, resonant absorption. Phys. Rev. Lett. 59 no. 1, pp. $52-55$ (1987).

[100] F. Brunel. Harmonic generation due to plasma effects in a gas undergoing multiphoton ionization in the high-intensity limit. J. Opt. Soc. Am. B 7 no. 4, pp. 521-526 (1990).

[101] P. B. Corkum, N. H. Burnett and F. Brunel. Above-threshold ionization in the long-wavelength limit. Phys. Rev. Lett. 62 no. 11, pp. 1259-1262 (1989).

[102] F. Ehlotzky. Harmonic generation in keldysh-type models. Nuovo Cimento D 14 no. 5, pp. 517-525 (1992).

[103] P. B. Corkum. Plasma perspective on strong field multiphoton ionization. Phys. Rev. Lett. 71 no. 13, pp. 1994-1997 (1993).

[104] K. C. Kulander, K. J. Schafer and J. L. Krause. Dynamics of short-pulse excitation, ionization and harmonic conversion. In B. Piraux, A. L'Huillier and K. Rzążewski (eds.), Super-Intense Laser Atom Physics, vol. 316 of NATO Advanced Studies Institute Series B: Physics, pp. 95-110 (Plenum, New York, 1993).

[105] The workshop was famous also for the fact that there was a flood of the river and there was no fresh water, so that the participants had to fight their thirst by drinking Belgian beers in large quantities.

[106] A. L'Huillier, M. Lewenstein, P. Salières et al. High-order harmonic-generation cutoff. Phys. Rev. A 48 no. 5, pp. R3433-R3436 (1993).

[107] M. Lewenstein, P. Balcou, M. Y. Ivanov, A. L'Huillier and P. B. Corkum. Theory of high-harmonic generation by low-frequency laser fields. Phys. Rev. A 49 no. 3, pp. 2117-2132 (1994).

[108] B. Yang, K. J. Schafer, B. Walker et al. Intensity-dependent scattering rings in high order above-threshold ionization. Phys. Rev. Lett. 71 no. 23, pp. 3770-3773 (1993).

[109] W. Becker, S. Long and J. K. McIver. Modeling harmonic generation by a zero-range potential. Phys. Rev. A 50 no. 2, pp. 1540-1560 (1994).

[110] N. Suárez, A. Chacón, M. F. Ciappina, J. Biegert and M. Lewenstein. Above-threshold ionization and photoelectron spectra in atomic systems driven by strong laser fields. Phys. Rev. A 92, p. 063421 (2015). arXiv:1509.01929.

[111] N. Suárez, A. Chacón, M. F. Ciappina et al. Above-threshold ionization and laser-induced electron diffraction in diatomic molecules. Phys. Rev. A 94 no. 4, p. 043423 (2016). arXiv:1607.07560.

[112] N. Suárez, A. Chacón, J. A. Pérez-Hernández et al. High-order-harmonic generation in atomic and molecular systems. Phys. Rev. A 95 no. 3, p. 033415 (2017). arXiv:1701.05021.

[113] N. Suárez, A. Chacón, E. Pisanty et al. Above-threshold ionization in multicenter molecules: the role of the initial state. Phys. Rev. A 97 no. 3, p. 033415 (2018). arXiv:1709.04366.

[114] N. Suárez. Strong Field Processes in Atoms and Polyatomic Molecules. PhD thesis, ICFO, Barcelona (2018).

[115] F. H. Faisal. Exact solution of the general separable potential model of a quantum system in an electromagnetic field. Phys. Lett. A 119 no. 8, pp. 375-378 (1987). 
[116] F. H. Faisal. Gauge-invariant exact solution of a general separable potential model of a quantum system in a laser field. Phys. Lett. A 125 no. 4, pp. 200-204 (1987).

[117] F. Faisal, P. Scanzana and J. Zarembo. Analytical ati spectrum of an exactly solvable 3d model of laser-atom interaction. J. Phys. B: At. Mol. Opt. Phys. 22 no. 8, p. L183 (1989).

[118] F. H. M. Faisal. Unified theory of above threshold ionization, multi-harmonic radiation and radiative electron scattering in strong laser fields. In C. A. Nicolaides, C. W. Clark and M. H. Nayfeh (eds.), Atoms in Strong Fields, vol. 212 of NATO Advanced Studies Institute Series B: Physics, pp. 407-424 (Springer, Boston, 1990).

[119] F. Faisal and P. Scanzano. Solution of the initial-value problem in laser-atom interaction: a variational fourier transform method. Comput. Phys. Commun. 63 no. 1-3, pp. 265-278 (1991).

[120] F. H. M. Faisal and T. Radożycki. Three-dimensional relativistic model of a bound particle in an intense laser field. Phys. Rev. A 47 no. 5, pp. 4464-4473 (1993).

[121] F. H. M. Faisal and T. Radożycki. Three-dimensional relativistic model of a bound particle in an intense laser field. II. Phys. Rev. A 48 no. 1, pp. 554-557 (1993).

[122] T. Radożycki and F. H. M. Faisal. Multiphoton ejection of strongly bound relativistic electrons in very intense laser fields. Phys. Rev. A 48 no. 3, pp. 2407-2412 (1993).

[123] J. Z. Kamiński. On exactly soluble models for multiphoton processes. Czech. J. Phys. 39 no. 10, pp. 1061-1065 (1989).

[124] M. Klaiber, K. Z. Hatsagortsyan and C. H. Keitel. Above-threshold ionization beyond the dipole approximation. Phys. Rev. A 71 no. 3, p. 033408 (2005). UH eprint.

[125] H. Tetchou Nganso, S. Giraud, B. Piraux, Y. V. Popov and M. K. Njock. On the role of the Coulomb potential in strong field atomic ionization dynamics. J. Electron Spectrosc. 161 no. 1-3, pp. 178-181 (2007). ICTP eprint.

[126] H. M. Tetchou Nganso, Y. V. Popov, B. Piraux, J. Madroñero and M. G. K. Njock. Ionization of atoms by strong infrared fields: solution of the time-dependent schrödinger equation in momentum space for a model based on separable potentials. Phys. Rev. A 83 no. 1, p. 013401 (2011).

[127] H. M. Tetchou Nganso, A. Hamido, M. G. Kwato Njock, Y. V. Popov and B. Piraux. Interaction of a model atom exposed to strong laser pulses: role of the Coulomb potential. Phys. Rev. A 87 no. 1, p. 013420 (2013). UC Louvain eprint.

[128] A. Galstyan, Y. V. Popov, F. Mota-Furtado et al. Modelling laser-atom interactions in the strong field regime. Eur. Phys. J. D 71 no. 4, p. 97 (2017). arXiv:1611.04300.

[129] A. Galstyan, Y. V. Popov, N. Janssens et al. Ionisation of $\mathrm{H}_{2} \mathrm{O}$ by a strong ultrashort XUV pulse: a model within the single active electron approximation. Chem. Phys. 504, pp. 22-30 (2018). arXiv:1703.05517.

[130] P. Salières, A. L'Huillier and M. Lewenstein. Coherence control of high-order harmonics. Phys. Rev. Lett. 74 no. 19, pp. 3776-3779 (1995).

[131] M. Lewenstein, P. Salières and A. L'Huillier. Phase of the atomic polarization in high-order harmonic generation. Phys. Rev. A 52 no. 6, pp. 4747-4754 (1995).

[132] P. Antoine, A. L'Huillier and M. Lewenstein. Attosecond pulse trains using high-order harmonics. Phys. Rev. Lett. 77 no. 7, pp. 1234-1237 (1996).

[133] P. M. Paul, E. S. Toma, P. Breger et al. Observation of a train of attosecond pulses from high harmonic generation. Science 292 no. 5522, pp. 1689-1692 (2001).

[134] M. Hentschel, R. Kienberger, C. Spielmann et al. Attosecond metrology. Nature 414 no. 6863 , p. 509 (2001).

[135] R. Kienberger, E. Goulielmakis, M. Uiberacker et al. Atomic transient recorder. Nature 427 no. 6977 , p. 817 (2004).

[136] G. Sansone, E. Benedetti, F. Calegari et al. Isolated single-cycle attosecond pulses. Science 314 no. 5798, pp. $443-446$ (2006).

[137] P. Salières, B. Carré, L. Le Déroff et al. Feynman's path-integral approach for intense-laser-atom interactions. Science 292 no. 5518, pp. 902-905 (2001).

[138] W. Becker, X. Liu, P. J. Ho and J. H. Eberly. Theories of photoelectron correlation in laser-driven multiple atomic ionization. Rev. Mod. Phys. 84 no. 3, pp. 1011-1043 (2012).

[139] H. Reiss. Properties of the strong-field approximation (2018). arXiv:1810.12106.

[140] A. Rudenko, K. Zrost, T. Ergler et al. Coulomb singularity in the transverse momentum distribution for strong-field single ionization. J. Phys. B: At. Mol. Opt. Phys. 38 no. 11, p. L191 (2005).

[141] M. Bashkansky, P. H. Bucksbaum and D. W. Schumacher. Asymmetries in above-threshold ionization. Phys. Rev. Lett. 60 no. 24, pp. 2458-2461 (1988).

[142] C. I. Blaga, F. Catoire, P. Colosimo et al. Strong-field photoionization revisited. Nature Phys. 5 no. 5 , pp. 335-338 (2009). OSU e-print.

[143] D. B. Milošević and F. Ehlotzky. Coulomb and rescattering effects in above-threshold ionization. Phys. Rev. A 58 no. 4 , pp. 3124-3127 (1998).

[144] D. G. Arbó, J. E. Miraglia, M. S. Gravielle et al. Coulomb-Volkov approximation for near-threshold ionization by short laser pulses. Phys. Rev. A 77 no. 1, p. 013401 (2008).

[145] L. Torlina and O. Smirnova. Time-dependent analytical $R$-matrix approach for strong-field dynamics. I. One-electron systems. Phys. Rev. A 86 no. 4, p. 043408 (2012).

[146] S. Popruzhenko and D. Bauer. Strong field approximation for systems with Coulomb interaction. J. Mod. Opt. 55 no. 16, pp. 2573-2589 (2008). arXiv:0803.1972.

[147] T.-M. Yan, S. V. Popruzhenko, M. J. J. Vrakking and D. Bauer. Low-energy structures in strong field ionization revealed by quantum orbits. Phys. Rev. Lett. 105 no. 25, p. 253002 (2010).

[148] O. Smirnova, M. Spanner and M. Ivanov. Analytical solutions for strong field-driven atomic and molecular one- and two-electron continua and applications to strong-field problems. Phys. Rev. A 77 no. 3, p. 033407 (2008). 
[149] X.-Y. Lai, C. Poli, H. Schomerus and C. Figueira de Morisson Faria. Influence of the Coulomb potential on abovethreshold ionization: a quantum-orbit analysis beyond the strong-field approximation. Phys. Rev. A 92 no. 4 , p. 043407 (2015).

[150] A. S. Maxwell, A. Al-Jawahiry, T. Das and C. Figueira de Morisson Faria. Coulomb-corrected quantum interference in above-threshold ionization: working towards multitrajectory electron holography. Phys. Rev. A 96 no. 2, p. 023420 (2017). arXiv:1705.01518.

[151] A. Maxwell and C. Figueira de Morisson Faria. Coulomb-free and Coulomb-distorted recolliding quantum orbits in photoelectron holography. J. Phys. B: At. Mol. Opt. Phys. 51 no. 12, p. 124001 (2018).

[152] V. Mosert and D. Bauer. Photoelectron spectra with Qprop and t-SURFF. Comput. Phys. Commun. 207, pp. 452-463 (2016). arXiv:1603.05529.

[153] A. Maxwell, A. Al-Jawahiry, X. Lai and C. Figueira de Morisson Faria. Analytic quantum-interference conditions in Coulomb corrected photoelectron holography. J. Phys. B: At. Mol. Opt. Phys. 51 no. 4, p. 044004 (2018).

[154] R. Kopold, D. B. Milošević and W. Becker. Rescattering processes for elliptical polarization: a quantum trajectory analysis. Phys. Rev. Lett. 84 no. 17, pp. 3831-3834 (2000).

[155] R. Kopold, W. Becker and D. B. Milošević. Quantum orbits: a space-time picture of intense-laser-induced processes in atoms. J. Mod. Opt. 49 no. 12, pp. 1987-1999 (2002).

[156] C. Figueira de Morisson Faria, D. B. Milošević and G. G. Paulus. Phase-dependent effects in bichromatic high-order harmonic generation. Phys. Rev. A 61 no. 6, p. 063415 (2000).

[157] G. Sansone, E. Benedetti, J.-P. Caumes et al. Control of long electron quantum paths in high-order harmonic generation by phase-stabilized light pulses. Phys. Rev. A 73 no. 5, p. 053408 (2006).

[158] A. Zaïr, M. Holler, A. Guandalini et al. Quantum path interferences in high-order harmonic generation. Phys. Rev. Lett. 100 no. 14, p. 143902 (2008).

[159] C. Figueira de Morisson Faria, H. Schomerus and W. Becker. High-order above-threshold ionization: the uniform approximation and the effect of the binding potential. Phys. Rev. A 66 no. 4, p. 043413 (2002).

[160] D. B. Milošević and W. Becker. Role of long quantum orbits in high-order harmonic generation. Phys. Rev. A 66 no. 6 , p. 063417 (2002).

[161] J. A. Pérez-Hernández, L. Roso and L. Plaja. Harmonic generation beyond the Strong-Field Approximation: the physics behind the short-wave-infrared scaling laws. Opt. Express 17 no. 12, pp. 9891-9903 (2009).

[162] Y. Huismans, A. Rouzée, A. Gijsbertsen et al. Time-resolved holography with photoelectrons. Science 331 no. 6013 , pp. 61-64 (2011). HAL e-print.

[163] T. Weber, H. Giessen, M. Weckenbrock et al. Correlated electron emission in multiphoton double ionization. Nature 405 no. 6787 , p. 658 (2000).

[164] C. Figueira de Morisson Faria and X. Liu. Electron-electron correlation in strong laser fields. J. Mod. Opt. 58 no. 13, pp. 1076-1131 (2011).

[165] J. S. Parker, B. J. S. Doherty, K. T. Taylor et al. High-energy cutoff in the spectrum of strong-field nonsequential double ionization. Phys. Rev. Lett. 96 no. 13, p. 133001 (2006). OSU eprint.

[166] D. F. Ye, X. Liu and J. Liu. Classical trajectory diagnosis of a fingerlike pattern in the correlated electron momentum distribution in strong field double ionization of helium. Phys. Rev. Lett. 101 no. 23, p. 233003 (2008).

[167] A. Emmanouilidou. Recoil collisions as a portal to field-assisted ionization at near-uv frequencies in the strong-field double ionization of helium. Phys. Rev. A 78 no. 2, p. 023411 (2008). arXiv:0805.4117.

[168] E. Eremina, X. Liu, H. Rottke et al. Laser-induced non-sequential double ionization investigated at and below the threshold for electron impact ionization. J. Phys. B: At. Mol. Opt. Phys. 36 no. 15, p. 3269 (2003). MPG eprint.

[169] S. L. Haan, Z. S. Smith, K. N. Shomsky, P. W. Plantinga and T. L. Atallah. Anticorrelated electrons from high-intensity nonsequential double ionization of atoms. Phys. Rev. A 81 no. 2, p. 023409 (2010). CC eprint.

[170] B. Bergues, M. Kübel, N. G. Johnson et al. Attosecond tracing of correlated electron-emission in non-sequential double ionization. Nature Commun. 3, p. 813 (2012).

[171] X. Sun, M. Li, D. Ye et al. Mechanisms of strong-field double ionization of xe. Phys. Rev. Lett. 113 no. 10 , p. 103001 (2014).

[172] M. Kübel, K. Betsch, N. G. Kling et al. Non-sequential double ionization of Ar: from the single-to the many-cycle regime. New J. Phys. 16 no. 3, p. 033008 (2014).

[173] D. F. Ye and J. Liu. Strong-field double ionization at the transition to below the recollision threshold. Phys. Rev. A 81 no. 4, p. 043402 (2010).

[174] A. Emmanouilidou and A. Staudte. Intensity dependence of strong-field double-ionization mechanisms: from field-assisted recollision ionization to recollision-assisted field ionization. Phys. Rev. A 80 no. 5, p. 053415 (2009).

[175] T. Shaaran, M. T. Nygren and C. Figueira de Morisson Faria. Laser-induced nonsequential double ionization at and above the recollision-excitation-tunneling threshold. Phys. Rev. A 81 no. 6, p. 063413 (2010).

[176] Z. Chen, Y. Liang and C. D. Lin. Quantitative rescattering theory of correlated two-electron momentum spectra for strong-field nonsequential double ionization of helium. Phys. Rev. A 82 no. 6, p. 063417 (2010).

[177] X. Hao, J. Chen, W. Li et al. Quantum effects in double ionization of argon below the threshold intensity. Phys. Rev. Lett. 112 no. 7, p. 073002 (2014).

[178] T. Shaaran, C. Figueira de Morisson Faria and H. Schomerus. Causality and quantum interference in time-delayed laser-induced nonsequential double ionization. Phys. Rev. A 85 no. 2, p. 023423 (2012).

[179] A. S. Maxwell and C. Figueira de Morisson Faria. Quantum interference in time-delayed nonsequential double ionization. Phys. Rev. A 92 no. 2, p. 023421 (2015). 
[180] W. Quan, X. Hao, Y. Wang et al. Quantum interference in laser-induced nonsequential double ionization. Phys. Rev. A 96 no. 3, p. 032511 (2017).

[181] S. Ghimire, A. D. DiChiara, E. Sistrunk et al. Observation of high-order harmonic generation in a bulk crystal. Nature Phys. 7 no. 2, p. 138 (2011). OSU eprint.

[182] G. Vampa, T. Hammond, N. Thiré et al. Linking high harmonics from gases and solids. Nature 522 no. 7557 , p. 462 (2015). NRC eprint.

[183] G. Vampa, C. R. McDonald, G. Orlando et al. Theoretical analysis of high-harmonic generation in solids. Phys. Rev. Lett. 113 no. 7, p. 073901 (2014). NRC eprint.

[184] E. N. Osika, A. Chacón, L. Ortmann et al. Wannier-Bloch approach to localization in high-harmonics generation in solids. Phys. Rev. X 7 no. 2, p. 021017 (2017).

[185] F. Krausz and M. I. Stockman. Attosecond metrology: from electron capture to future signal processing. Nature Photon. 8 no. 3, p. 205 (2014). GSU eprint.

[186] H. Liu, Y. Li, Y. S. You et al. High-harmonic generation from an atomically thin semiconductor. Nature Phys. 13 no. 3 , p. 262 (2017). Stanford eprint.

[187] T. T. Luu and H. J. Wörner. Measurement of the Berry curvature of solids using high-harmonic spectroscopy. Nature Commun. 9 no. 1, p. 916 (2018).

[188] R. Dum, A. Sanpera, K.-A. Suominen et al. Wave packet dynamics with Bose-Einstein condensates. Phys. Rev. Lett. 80 no. 18, pp. 3899-3902 (1998). arXiv:quant-ph/9708009.

[189] F. Morales, M. Richter, S. Patchkovskii and O. Smirnova. Imaging the Kramers-Henneberger atom. Proc. Natl. Acad. Sci. U.S.A. 108 no. 41, pp. 16906-16911 (2011).

[190] M. Lewenstein, A. Sanpera and V. Ahufinger. Ultracold Atoms in Optical Lattices: Simulating Quantum Many-Body Systems (Oxford University Press, Oxford, 2012).

[191] S. Arlinghaus and M. Holthaus. Driven optical lattices as strong-field simulators. Phys. Rev. A 81 no. 6 , p. 063612 (2010). arXiv:1006.4067.

[192] D.-S. Lühmann, C. Weitenberg and K. Sengstock. Emulating molecular orbitals and electronic dynamics with ultracold atoms. Phys. Rev. X 5 no. 3, p. 031016 (2015).

[193] S. Sala, J. Förster and A. Saenz. Ultracold-atom quantum simulator for attosecond science. Phys. Rev. A 95 no. 1 , p. 011403 (2017). arXiv:1311.2304.

[194] S. V. Rajagopal, K. M. Fujiwara, R. Senaratne et al. Quantum emulation of extreme non-equilibrium phenomena with trapped atoms. Ann. Phys. 529 no. 8, p. 1700008 (2017).

[195] R. Ramos, D. Spierings, S. Potnis and A. M. Steinberg. Atom-optics knife edge: measuring narrow momentum distributions. Phys. Rev. A 98 no. 2, p. 023611 (2018). arXiv:1806.00068.

[196] R. Senaratne, S. V. Rajagopal, T. Shimasaki et al. Quantum simulation of ultrafast dynamics using trapped ultracold atoms. Nature Commun. 9 no. 1, p. 2065 (2018).

[197] M. Kahn and G. Marcus. Proposal for strong field physics simulation by means of optical waveguide. J. Phys. B At. Mol. Opt. Phys. 50 no. 9, p. 095004 (2017).

[198] J. L. Hansen. Imaging molecular frame dynamics using spatially oriented molecules. PhD thesis, Aarhus University (2012).

[199] C. Jin, H. J. Wörner, V. Tosa et al. Separation of target structure and medium propagation effects in high-harmonic generation. J. Phys. B: At. Mol. Opt. Phys. 44 no. 9, p. 095601 (2011). arXiv:1012.1892.

[200] B. Buades, D. Moonshiram, T. P. Sidiropoulos et al. Dispersive soft x-ray absorption fine-structure spectroscopy in graphite with an attosecond pulse. Optica 5 no. 5, pp. 502-506 (2018). arXiv:1802.04652.

[201] P. Corkum, N. Burnett and M. Y. Ivanov. Subfemtosecond pulses. Opt. Lett. 19 no. 22, pp. 1870-1872 (1994).

[202] M. Kovacev, Y. Mairesse, E. Priori et al. Temporal confinement of the harmonic emission through polarization gating. Eur. Phys. J. D 26 no. 1, pp. 79-82 (2003).

[203] H. Mashiko, S. Gilbertson, C. Li et al. Double optical gating of high-order harmonic generation with carrier-envelope phase stabilized lasers. Phys. Rev. Lett. 100 no. 10, p. 103906 (2008). UCF eprint.

[204] T. Pfeifer, A. Jullien, M. J. Abel et al. Generating coherent broadband continuum soft-x-ray radiation by attosecond ionization gating. Opt. Express 15 no. 25, pp. 17120-17128 (2007).

[205] H. Vincenti and F. Quéré. Attosecond lighthouses: how to use spatiotemporally coupled light fields to generate isolated attosecond pulses. Phys. Rev. Lett. 108 no. 11, p. 113904 (2012). arXiv:1108.5559.

[206] C. Heyl, C. Arnold, A. Couairon and A. LâĂŹHuillier. Introduction to macroscopic power scaling principles for high-order harmonic generation. J. Phys. B: At. Mol. Opt. Phys. 50 no. 1, p. 013001 (2016).

[207] M. G. Pullen, B. Wolter, X. Wang et al. Transition from nonsequential to sequential double ionization in many-electron systems. Phys. Rev. A 96 no. 3, p. 033401 (2017). arXiv:1602.07840.

[208] B. Feuerstein, R. Moshammer, D. Fischer et al. Separation of recollision mechanisms in nonsequential strong field double ionization of Ar: the role of excitation tunneling. Phys. Rev. Lett. 87 no. 4, p. 043003 (2001). MST eprint.

[209] B. Wolter, M. G. Pullen, A.-T. Le et al. Ultrafast electron diffraction imaging of bond breaking in di-ionized acetylene. Science 354 no. 6310, pp. 308-312 (2016). CORE eprint.

[210] M. G. Pullen, B. Wolter, A.-T. Le et al. Influence of orbital symmetry on diffraction imaging with rescattering electron wave packets. Nature Commun. 7, p. 11922 (2016).

[211] Y. Ito, C. Wang, A.-T. Le et al. Extracting conformational structure information of benzene molecules via laser-induced electron diffraction. Struct. Dyn. 3 no. 3, p. 034303 (2016). KSU eprint.

[212] K. Amini, M. Sclafani, T. Steinle et al. Imaging ultrafast skeletal deformations in polyatomic molecules using laser-induced 
electron diffraction (2018). arXiv:1805.06793.

[213] M. B. Gaarde, P. Antoine, A. L'Huillier, K. J. Schafer and K. C. Kulander. Macroscopic studies of short-pulse high-order harmonic generation using the time-dependent Schrödinger equation. Phys. Rev. A 57 no. 6, pp. 4553-4560 (1998).

[214] J. Tate, T. Auguste, H. G. Muller et al. Scaling of wave-packet dynamics in an intense midinfrared field. Phys. Rev. Lett. 98 no. 1, p. 013901 (2007).

[215] M. F. Ciappina, J. Biegert, R. Quidant and M. Lewenstein. High-order-harmonic generation from inhomogeneous fields. Phys. Rev. A 85 no. 3, p. 033828 (2012). arXiv:1110.0665.

[216] A. Scrinzi. Time-dependent Schrödinger equation. In T. Schultz and M. Vrakking (eds.), Attosecond and XUV Physics: Ultrafast Dynamics and Spectroscopy, pp. 257-292 (Wiley-VCH, Weinheim, 2014).

[217] K. C. Kulander. Multiphoton ionization of hydrogen: a time-dependent theory. Phys. Rev. A 35 no. 1, pp. 445-447 (1987).

[218] H. G. Muller. Numerical simulation of high-order above-threshold-ionization enhancement in argon. Phys. Rev. A 60 no. 2, pp. 1341-1350 (1999).

[219] D. Bauer. Emergence of classical orbits in few-cycle above-threshold ionization of atomic hydrogen. Phys. Rev. Lett. 94 no. 11, p. 113001 (2005). arXiv:physics/0501007.

[220] M. Ruf, H. Bauke and C. H. Keitel. A real space split operator method for the klein-gordon equation. J. Comput. Phys. 228 no. 24, pp. 9092-9106 (2009).

[221] D. Bauer, D. B. Milošević and W. Becker. Strong-field approximation for intense-laserâĂŞatom processes: the choice of gauge. Phys. Rev. A 72 no. 2, p. 023415 (2005). arXiv:quant-ph/0504053.

[222] A. Galstyan, O. Chuluunbaatar, A. Hamido et al. Reformulation of the strong-field approximation for light-matter interactions. Phys. Rev. A 93 no. 2, p. 023422 (2016). arXiv:1512.00681.

[223] A. Sanpera, J. B. Watson, M. Lewenstein and K. Burnett. Harmonic-generation control. Phys. Rev. A 54 no. 5, pp. 4320-4326 (1996).

[224] L. Elsgolts. Differential equations and the calculus of variations (University Press of the Pacific, Miami, 2003).

[225] N. Bleistein and R. A. Handelsman. Asymptotic Expansions of Integrals (Dover, New York, 1975).

[226] K. S. Budil, P. Salières, A. L'Huillier, T. Ditmire and M. D. Perry. Influence of ellipticity on harmonic generation. Phys. Rev. A 48 no. 5, pp. R3437-R3440 (1993).

[227] P. Antoine, A. L'Huillier, M. Lewenstein, P. Salières and B. Carré. Theory of high-order harmonic generation by an elliptically polarized laser field. Phys. Rev. A 53 no. 3, pp. 1725-1745 (1996). Lund eprint.

[228] P. Antoine, B. Carré, A. L'Huillier and M. Lewenstein. Polarization of high-order harmonics. Phys. Rev. A 55 no. 2, pp. 1314-1324 (1997). Lund eprint.

[229] A. Ferré, C. Handschin, M. Dumergue et al. A table-top ultrashort light source in the extreme ultraviolet for circular dichroism experiments. Nature Photon. 9 no. 2, p. 93 (2015).

[230] A. Ferré, A. Boguslavskiy, M. Dagan et al. Multi-channel electronic and vibrational dynamics in polyatomic resonant high-order harmonic generation. Nature Commun. 6, p. 5952 (2015).

[231] J. Levesque, Y. Mairesse, N. Dudovich et al. Polarization state of high-order harmonic emission from aligned molecules. Phys. Rev. Lett. 99, p. 243001 (2007).

[232] X. Zhou, R. Lock, N. Wagner et al. Elliptically polarized high-order harmonic emission from molecules in linearly polarized laser fields. Phys. Rev. Lett. 102 no. 7, p. 073902 (2009). JILA eprint.

[233] D. Shafir, B. Fabre, J. Higuet et al. Role of the ionic potential in high harmonic generation. Phys. Rev. Lett. 108 no. 20, p. 203001 (2012). arXiv:1301.4112.

[234] D. B. Milošević, W. Becker and R. Kopold. Generation of circularly polarized high-order harmonics by two-color coplanar field mixing. Phys. Rev. A 61 no. 6, p. 063403 (2000).

[235] A. Fleischer, O. Kfir, T. Diskin, P. Sidorenko and O. Cohen. Spin angular momentum and tunable polarization in high-harmonic generation. Nature Photon. 8 no. 7, pp. 543-549 (2014). arXiv:1310.1206.

[236] O. Kfir, patrik Grychtol, E. Turgut et al. Generation of bright phase-matched circularly-polarized extreme ultraviolet high harmonics. Nature Photon. 9 no. 2, pp. 99 - 105 (2015).

[237] E. Pisanty Alatorre. Electron dynamics in complex time and complex space. PhD thesis, Imperial College London (2016).

[238] K. Veyrinas, C. Elkharrat, S. Marggi Poullain et al. Complete determination of the state of elliptically polarized light by electron-ion vector correlations. Phys. Rev. A 88 no. 6, p. 063411 (2013).

[239] K. Veyrinas, V. Gruson, S. Weber et al. Molecular frame photoemission by a comb of elliptical high-order harmonics: a sensitive probe of both photodynamics and harmonic complete polarization state. Faraday Discuss. 194, pp. 161-183 (2016).

[240] L. Barreau, K. Veyrinas, V. Gruson et al. Evidence of depolarization and ellipticity of high harmonics driven by ultrashort bichromatic circularly polarized fields. Nature Commun. 9 no. 1, p. 4727 (2018).

[241] E. Pisanty, L. Rego, J. San RomÃąn et al. Conservation of torus-knot angular momentum in high-order harmonic generation (2018). arXiv:1810.06503.

[242] E. Pisanty, G. Jiménez, V. Vicuña et al. Knotting fractional-order knots with the polarization state of light (2018). arXiv:1808.05193.

[243] C. M. Maharjan, A. S. Alnaser, I. Litvinyuk, P. Ranitovic and C. L. Cocke. Wavelength dependence of momentum-space images of low-energy electrons generated by short intense laser pulses at high intensities. J. Phys. B At. Mol. Opt. Phys. 39 no. 8, p. 1955 (2006).

[244] A. S. Maxwell and C. Figueira de Morisson Faria. Controlling below-threshold nonsequential double ionization via quantum interference. Phys. Rev. Lett. 116 no. 14, p. 143001 (2016). 
[245] W. Li, X. Zhou, R. Lock et al. Time-resolved dynamics in $\mathrm{N}_{2} \mathrm{O}_{4}$ probed using high harmonic generation. Science 322 no. 5905, pp. 1207-1211 (2008). JILA eprint.

[246] H. J. Wörner, J. B. Bertrand, D. V. Kartashov, P. B. Corkum and D. M. Villeneuve. Following a chemical reaction using high-harmonic interferometry. Nature 466 no. 7306, p. 604 (2010). NRC eprint.

[247] M. Lein. Attosecond probing of vibrational dynamics with high-harmonic generation. Phys. Rev. Lett. 94 no. 5 , p. 053004 (2005). UH eprint.

[248] S. Baker, J. S. Robinson, C. Haworth et al. Probing proton dynamics in molecules on an attosecond time scale. Science 312 no. 5772, pp. 424-427 (2006).

[249] S. Patchkovskii. Nuclear dynamics in polyatomic molecules and high-order harmonic generation. Phys. Rev. Lett. 102 no. 25, p. 253602 (2009).

[250] P. Heissler, E. Lugovoy, R. Hörlein et al. Using the third state of matter: high harmonic generation from liquid targets. New J. Phys. 16 no. 11, p. 113045 (2014).

[251] T. T. Luu, Z. Yin, A. Jain et al. Extreme-ultraviolet high-harmonic generation in liquids. Nature Commun. 9 no. 1, p. 3723 (2018).

[252] G. Vampa and T. Brabec. Merge of high harmonic generation from gases and solids and its implications for attosecond science. J. Phys. B: At. Mol. Opt. Phys. 50 no. 8, p. 083001 (2017).

[253] A. Chacón, W. Zhu, S. P. Kelly et al. Observing topological phase transitions with high harmonic generation (2018). arXiv:1807.01616.

[254] P. G. Hawkins and M. Y. Ivanov. Role of subcycle transition dynamics in high-order-harmonic generation in periodic structures. Phys. Rev. A 87 no. 6, p. 063842 (2013).

[255] T. Higuchi, M. I. Stockman and P. Hommelhoff. Strong-field perspective on high-harmonic radiation from bulk solids. Phys. Rev. Lett. 113 no. 21, p. 213901 (2014). arXiv:1406.7234.

[256] K. von Klitzing. The quantized Hall effect. Rev. Mod. Phys. 58 no. 3, pp. 519-531 (1986). Nobel Prize lecture.

[257] F. D. M. Haldane. Nobel lecture: Topological quantum matter. Rev. Mod. Phys. 89 no. 4, p. 040502 (2017). Nobel Prize lecture.

[258] D. Xiao, M.-C. Chang and Q. Niu. Berry phase effects on electronic properties. Rev. Mod. Phys. 82 no. 3, pp. 1959-2007 (2010). arXiv:0907.2021.

[259] R. Silva, Á. Jiménez-Galán, B. Amorim, O. Smirnova and M. Ivanov. All optical ultrafast probe of a topological phase transition (2018). arXiv:1806.11232.

[260] X. M. Tong, Z. X. Zhao and C. D. Lin. Theory of molecular tunneling ionization. Phys. Rev. A 66 no. 3, p. 033402 (2002). KSU eprint.

[261] L. Pitaevskii and S. Stringari. Bose-Einstein condensation (Oxford University Press, Oxford, 2003).

[262] T. Busch, B.-G. Englert, K. Rzażewski and M. Wilkens. Two cold atoms in a harmonic trap. Found. Phys. 28 no. 4 , pp. 549-559 (1998).

\section{Appendix A: Time dependent ADK rates}

This is the first section in which we remind the reader about possible way to evaluate/estimate the amplitude of the ground state, $a(t)$, which is the essential ingredient of our formulation of the SFA. The ADK rates apply in principle for arbitrary atoms, but the theory can be extended to molecules [260]. This approach can be used for pulses which are not too short: the rate has to have time to "define itself". The static expressions for the ADK rates are

$$
W_{\mathrm{ADK}}=\left|C_{n^{*} l^{*}}\right|^{2} \sqrt{\frac{6}{\pi}} f_{l m} I_{p}\left(2\left(2 I_{p}\right)^{3 / 2} / F\right)^{2 n^{*}-|m|-3 / 2} \exp \left(-2\left(2 I_{p}\right)^{3} / 2 / 3 F\right),
$$

where $F$ is the peak value of the laser electric field, $n^{*}=Z / \sqrt{I_{p}}$, and $Z$ being the charge of the atomic/ionic core. The other symbols are

$$
\left|C_{n^{*} l^{*}}\right|^{2}=\frac{2^{2 n^{*}}}{n^{*} \Gamma\left(n^{*}+l^{*}+1\right) \Gamma\left(n^{*}-l^{*}\right)},
$$

with $\Gamma(\cdot)$ denoting the Gamma function, $l^{*}=n^{*}-1$, and

$$
f_{l m}=\frac{(2 l+1)(l+|m|) !}{2^{\mid} m|| m \mid !(l-|m|) !}
$$

with $l, m$ denoting electrons initial orbital and magnetic quantum numbers.

For shorter pulses, we obtain $W_{\mathrm{ADK}}(t)$ replacing $F \rightarrow F(t)=\mathcal{E}_{0} f(t)$ (the rates change adiabatically with the pulse envelope). If the laser frequency is even smaller, and the pulse shortened we can even replace $F \rightarrow F(t)=|\mathbf{E}(t)|$, i.e. the actual value of the electric field. 


\section{Appendix B: Ground state amplitude according to SFA}

Inserting the expression (14) in the equation for $a(t)$, we obtain

$$
\frac{d a(t)}{d t}=\int_{0}^{t} d t^{\prime} \gamma\left(t, t^{\prime}\right) a\left(t^{\prime}\right)
$$

where

$$
\begin{aligned}
\gamma\left(t, t^{\prime}\right)= & \int d^{3} \mathbf{p} \mathbf{E}(t) \cdot \mathbf{d}(\mathbf{p}-e \mathbf{A}(t) / c) \mathbf{E}\left(t^{\prime}\right) \cdot \mathbf{d}\left(\mathbf{p}-e \mathbf{A}\left(t^{\prime}\right) / c\right) \\
& \times \exp \left(-i \int_{t^{\prime}}^{t} d t^{\prime \prime}\left[\left(\mathbf{p}-e \mathbf{A}\left(t^{\prime \prime}\right) / c\right)^{2} / 2+I_{p}\right] / \hbar\right) .
\end{aligned}
$$

In principle solving equation (B1) in the static, quenched or even self-consistent case presents no basic difficulties. If the pulse is longer, and the rate of change of $a(t)$ slow, we may replace $a\left(t^{\prime}\right) \rightarrow a(t)$ and obtain explicitly

$$
a(t)=\exp \left(-W_{\mathrm{SFA}}(t)\right) a(0)
$$

where the SFA rate is

$$
W_{\mathrm{SFA}}=\int_{0}^{t} \int_{0}^{t^{\prime}} \gamma\left(t, t^{\prime}\right)
$$

\section{Appendix C: Model atom and molecule}

The paradigm examples of separable potentials used in atomic physics are zero-range potentials. In 1D, a Dirac delta potential can be used fr this purpose. In 3D, the Dirac delta must be regularized - that is why the celebrated pseudo-potential must be used. It has found multiple applications in the many-body theory of ultracold atomic gases (cf. Refs. 190, 261); in strong-field physics it was elaborated by W. Becker and his collaborators [109]. In 2D the situation is more complex due to the logarithmic divergencies (cf. Ref. 262 and references therein).

One should stress, however, that the use of separable potentials has a long history in strong-field physics [115-129]. Many of these papers deal with zero-range models, some with general separable potentials, but typically using laser fields of constant strength and circular polarization. For such a case, one can transform the problem to a rotating frame in which the Hamiltonian is time-indenpendent. The recent papers by Galstyan et al. [128, 129] are perhaps the closest to the approach developed by us in Refs. 110-114.

In this section we discuss a useful non-local separable potential with the purpose of applications for atoms, but most importantly for large molecules. The idea will be to compute both the direct and the re-scattering transition amplitudes [29]. These terms involve the dipole and the continuum-continuum matrix elements defined by Eqs. (11) and (12). Then, our main task will be devoted to find analytically the wavefunctions for the ground and scattering states of our model potential. The Hamiltonian, $\hat{H}\left(\mathbf{p}, \mathbf{p}^{\prime}\right)$, of the atomic system in the momentum representation can be written as:

$$
\hat{H}\left(\mathbf{p}, \mathbf{p}^{\prime}\right)=\frac{\mathbf{p}^{2}}{2} \delta\left(\mathbf{p}-\mathbf{p}^{\prime}\right)+\hat{V}\left(\mathbf{p}, \mathbf{p}^{\prime}\right),
$$

where the first term on the right-hand side is the kinetic energy operator, and the second one is the non-local potential $\hat{V}\left(\mathbf{p}, \mathbf{p}^{\prime}\right)$. We use non-local separable potentials that can be understood as sums of projectors on certain states. They generally have the form

$$
\hat{V}\left(\mathbf{p}, \mathbf{p}^{\prime}\right)=-\gamma \sum_{i=1}^{M} \phi_{i}(\mathbf{p}) \phi_{\mathbf{i}}^{*}\left(\mathbf{p}^{\prime}\right) .
$$

When we model molecules, each of the orbitals is typically centered in real space at the positions of the nuclei, $\mathbf{R}_{i}$. In the momentum representation it translates to $\phi_{i}(\mathbf{p}) \exp \left(\mathbf{i p} \cdot \mathbf{R}_{\mathbf{i}} \tilde{\phi}_{\mathbf{i}}(\mathbf{p})\right.$, where $\tilde{\phi}_{i}(\mathbf{p})$ is a "smooth" function, with the Fourier transform centered at $\mathbf{R}=0$. The above potential has generically $M$ bound states, so one can model with its help not only the ground state of the molecule in question, but even some of its excited states. Alternatively it may be used to model multielectron molecules. 


\section{Ground state}

In the present Appendix we will consider $M=1$ only, so the models with a single bound state, but we will explore the full richness of the orbital $\phi(\mathbf{p})$ to model multicentered ground states of, in principle, arbitrary molecules. By using the non-local separable Hamiltonian, we write the stationary Schrödinger equation as follows:

$$
E \Psi(\mathbf{p})=\frac{p^{2}}{2} \Psi(\mathbf{p})-\gamma \phi(\mathbf{p}) \int d^{3} \mathbf{p}^{\prime} \phi^{*}\left(\mathbf{p}^{\prime}\right) \Psi\left(\mathbf{p}^{\prime}\right),
$$

where $E$ denotes the energy of the wavefunction $\Psi(\mathbf{p})$. Note that we have defined the non-local potential as $\hat{V}\left(\mathbf{p}, \mathbf{p}^{\prime}\right)=$ $-\gamma \phi(\mathbf{p}) \phi\left(\mathbf{p}^{\prime}\right)$, which describes the attraction between the electron and the nucleus [29]. This potential has been chosen such that it assures analytical solutions of the continuum or scattering states, i.e. for states with energies $E>0$. Note that the ground state can also be calculated analytically. The parameter $\gamma$ is a constant that, as we will see, determines the energy of the ground state. The shape of the ground state, however, can be controlled to a high degree by the choice of a suitable auxiliary function $\phi(\mathbf{p})$, which may correspond to a multicenter molecular orbital of arbitrary shape.

For the ground state, $\Psi_{0}(\mathbf{p})$, we solve the stationary Schrödinger equation in the momentum representation:

$$
\frac{p^{2}}{2} \Psi_{0}(\mathbf{p})-\gamma \phi(\mathbf{p}) \int d^{3} \mathbf{p}^{\prime} \phi^{*}\left(\mathbf{p}^{\prime}\right) \Psi_{0}\left(\mathbf{p}^{\prime}\right)=-I_{p} \Psi_{0}(\mathbf{p}),
$$

From Eq. (C4) we easily determine the ground state:

$$
\Psi_{0}(\mathbf{p})=\frac{\mathcal{N} \phi(\mathbf{p})}{\left(\frac{p^{2}}{2}+I_{p}\right)}
$$

where, $\mathcal{N}$ denotes a normalization constant. Multiplying the last formula by $\phi(\mathbf{p})$, and taking the volume integral on $\mathbf{p}$, we obtain equation that determines the ground state energy,

$$
1=\gamma \int \frac{d^{3} \mathbf{p}|\phi(\mathbf{p})|^{2}}{\left(\frac{p^{2}}{2}+I_{p}\right)} .
$$

The solution of the last integral in Eq. (C6) gives us the relation between the parameters $I_{p}$, and $\gamma$ given $\phi(\mathbf{p})$. The normalization constant fulfils

$$
1=\mathcal{N}^{2} \int \frac{d^{3} \mathbf{p}|\phi(\mathbf{p})|^{2}}{\left(\frac{p^{2}}{2}+I_{p}\right)^{2}}
$$

\section{Scattering waves}

Let us consider the scattering wave, $\Psi_{\mathbf{p}_{0}}(\mathbf{p})$, with asymptotic momentum $\mathbf{p}_{0}$, as a coherent superposition of a plane wave and an extra correction $\delta \Psi_{\mathbf{p}_{0}}(\mathbf{p})$ :

$$
\Psi_{\mathbf{p}_{0}}(\mathbf{p})=\delta\left(\mathbf{p}-\mathbf{p}_{0}\right)+\delta \Psi_{\mathbf{p}_{0}}(\mathbf{p})
$$

This state has an energy $E=\mathbf{p}_{0}^{2} / 2$. Then, the Schrödinger equation in momentum representation reads:

$$
\begin{aligned}
\frac{p_{0}^{2}}{2} \Psi_{\mathbf{p}_{0}}(\mathbf{p}) & =\frac{p^{2}}{2} \Psi_{\mathbf{p}_{0}}(\mathbf{p})-\gamma \phi(\mathbf{p}) \int d^{3} \mathbf{p}^{\prime} \phi^{*}\left(\mathbf{p}^{\prime}\right) \Psi_{\mathbf{p} 0}(\mathbf{p}), \\
\left(\frac{p^{2}}{2}-\frac{p_{0}^{2}}{2}\right) \delta \Psi_{\mathbf{p}_{0}}(\mathbf{p}) & =\gamma \phi(\mathbf{p}) \phi^{*}\left(\mathbf{p}_{0}\right)+\gamma \phi(\mathbf{p}) \int d^{3} \mathbf{p}^{\prime} \phi^{*}\left(\mathbf{p}^{\prime}\right) \delta \Psi_{\mathbf{p}_{0}}\left(\mathbf{p}^{\prime}\right) .
\end{aligned}
$$

To solve analytically the last equation, we apply elementary algebra and the following Dirac delta distribution properties: $\left(\frac{p^{2}}{2}-\frac{p_{0}^{2}}{2}\right) \delta\left(\mathbf{p}-\mathbf{p}_{0}\right)=0$, and $\left(\frac{p^{2}}{2}-\frac{p_{0}^{2}}{2}\right) \delta\left(\frac{p^{2}}{2}-\frac{p_{0}^{2}}{2} \pm i \epsilon\right)=0$. Finally, the correction $\delta \Psi_{\mathbf{p}_{0}}$ reads:

$$
\delta \Psi_{\mathbf{p}_{0}}(\mathbf{p})=\frac{2 \gamma \phi(\mathbf{p}) \phi^{*}\left(\mathbf{p}_{0}\right)}{\left(1-A\left(i p_{0}+\epsilon\right)\left(p^{2}-\left(p_{0}-i \epsilon\right)^{2}\right)\right.} .
$$


Here, $\epsilon$, is the (positive) regularization parameter to avoid the divergence at $\mathbf{p}=\mathbf{p}_{0}$. The sign in front of $\epsilon$ defines the asymptotic behaviour of the scattering solutions. For the present choice, the scattered part of the wavefunction in the position representation behaves asymptotically as $\exp \left(-i p_{0} r\right)$, i.e. as a incoming spherical wave, which is the correct behaviour for the scattering wave functions, describing the states with asymptotic outgoing momentum $\mathbf{p}_{0}$. The final expression for the scattering wave functions is:

$$
\Psi_{\mathbf{p}_{0}}(\mathbf{p})=\delta\left(\mathbf{p}-\mathbf{p}_{0}\right)+\frac{2 \gamma \phi(\mathbf{p}) \phi^{*}\left(\mathbf{p}_{0}\right)}{\left(1-A\left(i p_{0}+\epsilon\right)\left(p^{2}-\left(p_{0}-i \epsilon\right)^{2}\right)\right.} .
$$

\section{Dipole matrix element}

The dipole matrix element is

$$
\mathbf{d}\left(\mathbf{p}_{0}\right)=e\left\langle\Psi_{\mathbf{p}_{0}}\left|i \hbar \nabla_{\mathbf{p}}\right| \Psi_{0}\right\rangle
$$

Tedious, but elementary algebra leads to:

$$
\begin{aligned}
\mathbf{d}\left(\mathbf{p}_{0}\right) & =i e \mathcal{N}\left[\frac{\nabla_{\mathbf{p}} \phi(\mathbf{p})}{\left(p^{2}+2 I_{p}\right)}-\frac{\mathbf{p} \phi(\mathbf{p})}{\left(p^{2}+2 I_{p}\right)^{2}}\right] \\
& +2 i e \gamma \mathcal{N} \phi\left(\mathbf{p}_{0}\right)\left\{\int d^{3} \mathbf{p} \frac{\left[\left(\nabla_{\mathbf{p}} \phi(\mathbf{p})\right) \phi^{*}(\mathbf{p})+2 \mathbf{p}|\phi(\mathbf{p})|^{2}\right]}{\left(1-A\left(-i p_{0}+\epsilon\right)\right)\left(p^{2}+2 I_{p}\right)\left(p^{2}-\left(p_{0}-i \epsilon\right)^{2}\right)}\right\} .
\end{aligned}
$$

\section{Continuum-continuum transition matrix element}

Let us consider the scattering waves obtained in Eq. (C11) and evaluate the continuum-continuum transition matrix element of Eq. (12), i.e.

$$
e\left\langle\mathbf{p}_{1}|\mathbf{x}| \mathbf{p}_{2}\right\rangle=i e \hbar \nabla_{\mathbf{p}_{1}} \delta\left(\mathbf{p}_{1}-\mathbf{p}_{2}\right)+\hbar \mathbf{g}\left(\mathbf{p}_{1}, \mathbf{p}_{2}\right)
$$

Again after tedious (though straightforward) calculations we obtain:

$$
\begin{aligned}
& \mathbf{g}\left(\mathbf{p}_{1}, \mathbf{p}_{2}\right)=\left.2 i \gamma \nabla_{\mathbf{p}}\left[\frac{\phi(\mathbf{p}) \phi^{*}\left(\mathbf{p}_{2}\right)}{\left(1-A\left(i p_{0}+\epsilon\right)\right)\left(p^{2}+2 I_{p}\right)\left(\left(p^{2}-\left(p_{2}-i \epsilon\right)^{2}\right)\right.}\right]\right|_{\mathbf{p}=\mathbf{p}_{1}} \\
&-\left.2 i \gamma \nabla_{\mathbf{p}}\left[\frac{\phi^{*}(\mathbf{p}) \phi\left(\mathbf{p}_{1}\right)}{\left(1-A\left(-i p_{1}+\epsilon\right)\right)\left(p^{2}+2 I_{p}\right)\left(\left(p^{2}-\left(p_{1}+i \epsilon\right)^{2}\right)\right.}\right]\right|_{\mathbf{p}=\mathbf{p}_{2}} \\
&+4 \gamma^{2} \int d^{3} \mathbf{p}\left[\frac{\phi(\mathbf{p}) \phi^{*}\left(\mathbf{p}_{2}\right)}{\left(1-A\left(i p_{0}+\epsilon\right)\right)\left(p^{2}+2 I_{p}\right)\left(p^{2}-\left(p_{2}-i \epsilon\right)^{2}\right)}\right] . \\
& \nabla_{\mathbf{p}}\left[\frac{\phi^{*}(\mathbf{p}) \phi\left(\mathbf{p}_{1}\right)}{\left(1-A\left(-i p_{1}+\epsilon\right)\left(p^{2}+2 I_{p}\right)\left(p^{2}-\left(p_{1}+i \epsilon\right)^{2}\right)\right.}\right] .
\end{aligned}
$$

All of the above formulae are directly and easily generalized to the case when the Hamiltonian is time dependent.

\section{Appendix D: Dipole matrix elements}

In the remaining appendices, we turn to the objects of the two-electron theory and their properties.

\section{Dipole Matrix Elements from the ground state}

The dipole matrix element given by the function $\mathbf{d}$ deals with dipole transitions from the two electron ground state into all other admissible two electron states. This is the analogue of the function by the same name for one electron case, given by Eq. (11), but now there are three non-zero variants. The first, bearing the strongest resemblance to the one electron case, can be expressed as a sum of two terms

$$
\mathbf{d}(\mathbf{p})=e\left\langle 0\left|\hat{\mathbf{r}}_{1}\right| \mathbf{p}, 0\right\rangle+e\left\langle 0\left|\hat{\mathbf{r}}_{2}\right| \mathbf{p}, 0\right\rangle .
$$


Each term will have two contributions, (1) direct laser induced ionization of the electron acted on directly by the operator, while the other electron remains bound in a ground state and (2) correlated ionization where the the action of laser on one electron is transferred to the other via electron-electron interaction, this could be through elastic collision. This last contribution is expected to be small and will be zero in the non-interacting case, where Eq. (D1) simplifies to

$$
\begin{aligned}
\mathbf{d}(\mathbf{p}) & =\frac{e}{\sqrt{2}}\left(\left\langle 0\left|\left\langle 0\left|\hat{\mathbf{r}}_{1} \otimes \mathbb{I}_{2}\right| \mathbf{p}\right\rangle\right| 0\right\rangle+\left\langle 0\left|\left\langle 0\left|\mathbb{I}_{1} \otimes \hat{\mathbf{r}}_{2}\right| 0\right\rangle\right| \mathbf{p}\right\rangle\right) \\
& =\frac{2 e}{\sqrt{2}}\left\langle 0_{1}|\hat{\mathbf{r}}| \mathbf{p}\right\rangle
\end{aligned}
$$

The final expression is written in terms of 1-electron states and operators. This dipole matrix element is vital to most strong field processes as it will describe the initial tunnelling step, hence it is necessary to model NSDI for both the EI and RESI mechanism. The subscript denotes whether it is the first or second electron to be ionized, this is significant as the second will have a much larger ionization potential, which will make it less probable and for both mechanisms of NSDI we will neglect this contribution.

The other two variants of the $\mathbf{d}$ dipole matrix element require at least some electron interaction in all contributing processes and so will both be zero in the non-interacting case. The element $\mathbf{d}(\mathbf{p}, \eta)$ will again have two contributions, in the first the operator acts on an electron to ionize it and the electron-electron correlation causes the other electron to be excited in a 'shake-up' process. This would be the main term involved in the previously proposed shake-off mechanism for NSDI [79], which has since fallen out of favour in preference of the re-collision mechanisms EII and RESI. This will be the dominant of the two contributions. In the second (presumably less likely) transition, the laser excites an electron and the electron-electron correlation causes the second electron to be fully ionized. In both these possibilities the energy transfer between electron could be through direct collision or any other electron-electron interaction, however in the first only some of the electron's energy is transferred, while in the second scenario most of the electron's energy will be transferred to the other one.

For the dipole matrix element $\mathbf{d}\left(\mathbf{p}, \mathbf{p}^{\prime}\right)$ the only transition is one where the laser-induced ionization of an electron through interactions between the electrons causes the other electron to ionize as well-in fact, this matrix element, when dressed in the laser field, leads to collective tunnelling, as discussed in Ref. 56.

\section{Dipole Matrix Elements from the scattering and ground state}

The dipole matrix elements from the two electron scattering-ground state $|\mathbf{p}, 0\rangle$ are given by the function $\mathbf{g}$, there are three possible variants. The first, $\mathbf{g}\left(\mathbf{p}, \mathbf{p}^{\prime}\right)$ relates to the transition from the two electron continuum-ground state $|\mathbf{p}, 0\rangle$ to an alternative continuum-ground state $\left|\mathbf{p}^{\prime}, 0\right\rangle$. The leading contribution will typically come from the laser induced change of momentum of the continuum state, this term should play a strong role as we expect strong coupling between the laser and continuum electrons. Continuum-continuum transitions can, as in the one electron case, also involve contributions via interaction through the single electron potential. In Eq. (12) this was described by splitting of the continuum-continuum matrix element into two parts in the one-electron case. Alternatively, there is the strongly correlated and less likely process, where the laser acts on the bound electron which through electronelectron interaction changes the momentum of the scattering state for the other electron, without changing the state of the original bound electron. This would be quite an exotic case and generally it is a reasonable approximation to assume the two electrons in this state are some what physically separated. Of course, when an electron recollides with the it's parent atom/molecule then there will be much overlap and this term could contribute to elastic recollision processes such as high-order above-threshold ionization (HATI). In Fig. 12 the four pathways discussed are shown in the form of Feynman diagrams, the non-interacting cases are given by panels a) and b).

This dipole moment can be considerably simplified if we consider non-interacting electrons

$$
\mathbf{g}\left(\mathbf{p}, \mathbf{p}^{\prime}\right)=\frac{e}{2}\left(\left\langle\mathbf{p}\left|\left\langle 0|| \hat{\mathbf{r}}_{1}|| \mathbf{p}^{\prime}\right\rangle\right| 0\right\rangle+\left\langle 0\left|\left\langle\mathbf{p}|| \hat{\mathbf{r}}_{2}|| 0\right\rangle\right| \mathbf{p}^{\prime}\right\rangle\right)
$$

which can be written in terms of one particle state and operators as,

$$
\mathbf{g}_{1}\left(\mathbf{p}, \mathbf{p}^{\prime}\right)=e\left\langle\mathbf{p}|\hat{\mathbf{r}}| \mathbf{p}^{\prime}\right\rangle
$$

This can be treated as before by Eq. (12),

The dipole matrix element $\mathbf{g}\left(\mathbf{p}, \mathbf{p}^{\prime}, \eta\right)$ deals with transitions from $|\mathbf{p}, 0\rangle$ to $\left|\mathbf{p}^{\prime}, \eta\right\rangle$. This will have contributions from scattering states interacting with the laser to change the moment from $\mathbf{p}$ to $\mathbf{p}^{\prime}$, which simultaneously through the 

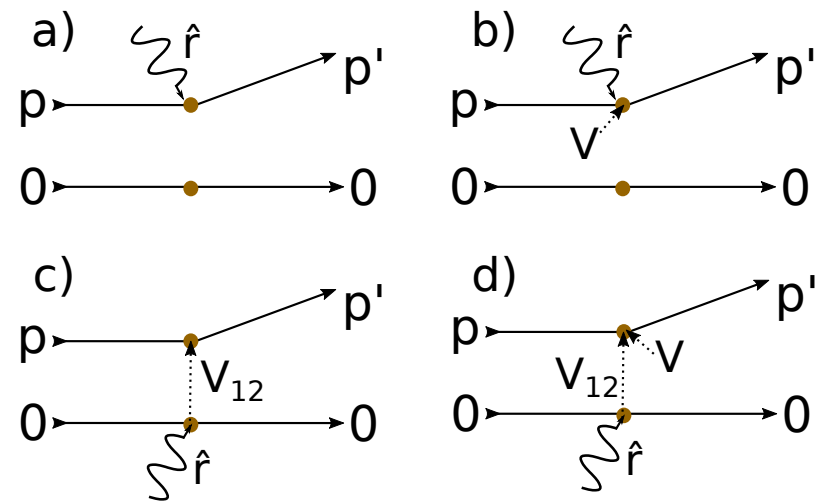

FIG. 12. Four main process for the matrix element $g\left(\mathbf{p}, \mathbf{p}^{\prime}\right)$, the top row [panels a) and b)] show processes where the laser dipole acts on the continuum state, the bottom row [panels c) and d)] show cases where the laser dipole acts on the ground state and electron interaction transfers the energy to the continuum state. The right hand column includes the continuum electrons interaction with the core potential
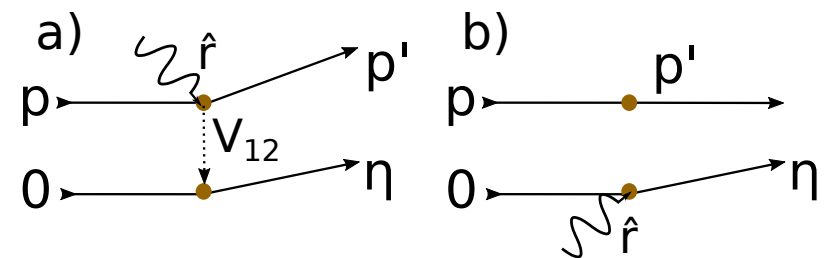

FIG. 13. Two process from the matrix element $g\left(\mathbf{p}, \mathbf{p}^{\prime}, \eta\right)$, panel a) shows the excitation process via electron interaction required in RESI, panel b) shows the laser induced excitation of the bound state while the continuum state remains unaffected.

electron interactions also leads to excitation of the other electron from it's ground state into an excited state with principle quantum number $\eta$. It is crucial to include this to allow for the excitation step of the second electron in the RESI mechanism of NSDI. Alternatively, there is the contribution that the excitation of an electron via the laser also results in the momentum change of the scattering state from $\mathbf{p}$ to $\mathbf{p}^{\prime}$. In the non-interacting case the first pathway is has no contribution as the ground state electron cannot be excited without electron correlation. The second pathway is non-zero in the case $\mathbf{p}=\mathbf{p}^{\prime}$ and the dipole matrix element can be written as

$$
\mathbf{g}\left(\mathbf{p}, \mathbf{p}^{\prime}, \eta\right)=e \delta\left(\mathbf{p}-\mathbf{p}^{\prime}\right)\langle 0|\hat{\mathbf{r}}| \eta\rangle
$$

The excitation via electron interaction and non-interacting pathways are depicted in panel a) and b) of Fig. 13, respectively.

It is a very similar situation for the dipole matrix element $\mathbf{g}\left(\mathbf{p}, \mathbf{p}^{\prime}, \mathbf{p}^{\prime \prime}\right)$, except the excited state is replaced by a scattering state with momentum $\mathbf{p}^{\prime \prime}$. There are the same kind of contributions, one where the change of momentum of the scattering states leads through electron-electron interaction to ionization of the bound state, this is the crucial ionization step of the second electron in the EI mechanism of NSDI. Another possibility is the laser induced ionization of the bound electron leads to change of momentum of the scattering state, which could happen through the collision of the two ionized electron. In the non-interacting case the first contribution is zero, while the second is non-zero again if $\mathbf{p}=\mathbf{p}^{\prime}$ which means the matrix element can be simplified to

$$
\mathbf{g}\left(\mathbf{p}, \mathbf{p}^{\prime}, \mathbf{p}^{\prime \prime}\right)=e \delta\left(\mathbf{p}-\mathbf{p}^{\prime}\right)\left\langle 0_{2}|\hat{\mathbf{r}}| \mathbf{p}^{\prime \prime}\right\rangle+e \delta\left(\mathbf{p}-\mathbf{p}^{\prime \prime}\right)\left\langle 0_{2}|\hat{\mathbf{r}}| \mathbf{p}^{\prime}\right\rangle
$$

The subscript two denotes that this is the laser induced ionization of a second electron, i.e. this electron comes from $\mathrm{a}+1$ ion, thus it is much less probably than the first ionization and for our purposes where we consider NSDI we will non consider such contributions. In Fig. 14 we show the EI recollision excitation pathway and also the non-interacting pathway, in panels a) and b), respectively.

\section{Dipole Matrix Elements from the scattering and excited states}

The dipole matrix elements from the two electron continuum-excited state $|\mathbf{p}, \eta\rangle$ are given by the function $\mathbf{h}$, there are two variants. The function $\mathbf{h}\left(\mathbf{p}, \eta, \mathbf{p}^{\prime}, \eta^{\prime}\right)$ deals with transitions to alternate continuum-excited states. This will 

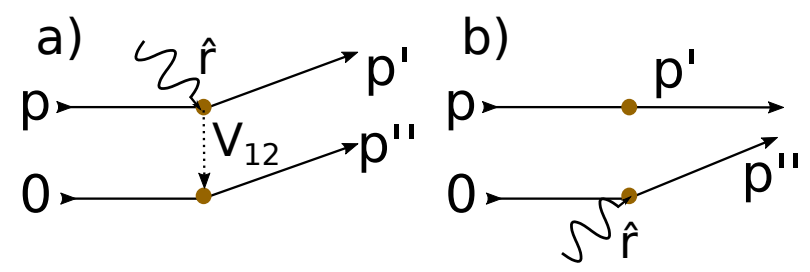

FIG. 14. Two process from the matrix element $g\left(\mathbf{p}, \mathbf{p}^{\prime}, \mathbf{p}^{\prime \prime}\right)$, panel a) shows the recollision ionization process from the EI of NSDI, panel b) shows the laser induced ionization of the bound state while the continuum state remains unaffected.

have contributions such as the laser induced recollision of the scattering state results in the excited electron moving to a another excited state. This will be non-zero in the non-interacting case if either $\mathbf{p}=\mathbf{p}^{\prime}$ or $\eta=\eta^{\prime}$ and the dipole matrix element can be written as

$$
\mathbf{h}\left(\mathbf{p}, \eta, \mathbf{p}^{\prime}, \eta^{\prime}\right)=e \delta_{\eta \eta^{\prime}}\left\langle\mathbf{p}|\hat{\mathbf{r}}| \mathbf{p}^{\prime}\right\rangle+e \delta\left(\mathbf{p}-\mathbf{p}^{\prime}\right)\left\langle\eta|\hat{\mathbf{r}}| \eta^{\prime}\right\rangle .
$$

The other function $\mathbf{h}\left(\mathbf{p}, \eta, \mathbf{p}^{\prime}, \mathbf{p}^{\prime \prime}\right)$ deals with transition to continuum-continuum state $\left|\mathbf{p}^{\prime}, \mathbf{p}^{\prime \prime}\right\rangle$. This will have a contribution where the laser induced recollision of the scattering state changes the momentum from $\mathbf{p}$ to $\mathbf{p}^{\prime}$ and the excited electron is ionized through electron-electron interaction. Alternatively, the laser induced ionization of the excited electron can change the momentum of the continuum electron through their interaction. The first contribution vanishes in the non-interacting case, while the second contribution is non-zero if $\mathbf{p}=\mathbf{p}^{\prime}$. This what leads to the final ionization step in the RESI mechanism of NSDI, where the excited electron tunnel ionizes via the dipole interaction. This dipole matrix element can be written as

$$
\mathbf{h}\left(\mathbf{p}, \eta, \mathbf{p}^{\prime}, \mathbf{p}^{\prime \prime}\right)=e \delta\left(\mathbf{p}-\mathbf{p}^{\prime \prime}\right)\left\langle\eta|\hat{\mathbf{r}}| \mathbf{p}^{\prime}\right\rangle+e \delta\left(\mathbf{p}-\mathbf{p}^{\prime}\right)\left\langle\eta|\hat{\mathbf{r}}| \mathbf{p}^{\prime \prime}\right\rangle
$$

\section{Dipole Matrix Elements from the scattering states}

This dipole matrix element is between two-electron scattering states. This will mostly contribute to the final evolution of the electrons before detection. It will have strong terms, where the laser induces a change in momentum in one electron and the other electron remains unaffected. But it also included the strongly correlated contribution, where the laser induces recollision/ interaction between the two electrons. The dipole matrix element in the noninteracting case is given by,

$$
\mathbf{i}\left(\mathbf{p}, \mathbf{p}^{\prime}, \mathbf{p}^{\prime}, \mathbf{p}^{\prime \prime}, \mathbf{p}^{\prime \prime \prime}\right)=e \delta\left(\mathbf{p}^{\prime}-\mathbf{p}^{\prime \prime \prime}\right)\left\langle\mathbf{p}|\hat{\mathbf{r}}| \mathbf{p}^{\prime \prime}\right\rangle+e \delta\left(\mathbf{p}^{\prime}-\mathbf{p}^{\prime \prime}\right)\left\langle\mathbf{p}|\hat{\mathbf{r}}| \mathbf{p}^{\prime \prime \prime}\right\rangle+e \delta\left(\mathbf{p}-\mathbf{p}^{\prime \prime \prime}\right)\left\langle\mathbf{p}^{\prime}|\hat{\mathbf{r}}| \mathbf{p}^{\prime \prime}\right\rangle+e \delta\left(\mathbf{p}-\mathbf{p}^{\prime \prime}\right)\left\langle\mathbf{p}^{\prime}|\hat{\mathbf{r}}| \mathbf{p}^{\prime \prime \prime}\right\rangle .
$$

\section{Appendix E: Two-Electron Integro-Differential Equations}

The time variations of $a(t), b(\mathbf{p}, t), c(\mathbf{p}, \eta, t)$ and $d\left(\mathbf{p}, \mathbf{p}^{\prime}, t\right)$ follow from TDSE for two electrons and read:

$$
\begin{aligned}
& \dot{a}(t)=-\frac{i}{\hbar}\left[\mathbf{E}(t) \cdot \int d^{3} \mathbf{p} b(\mathbf{p}, t) \mathbf{d}(\mathbf{p})+\mathbf{E}(t) \cdot \sum_{\eta \neq 0} \int d^{3} \mathbf{p} c(\mathbf{p}, \eta, t) \mathbf{d}(\mathbf{p}, \eta)\right.\left.+\mathbf{E}(t) \cdot \iint d^{3} \mathbf{p} d^{3} \mathbf{p}^{\prime} d\left(\mathbf{p}, \mathbf{p}^{\prime}, t\right) \mathbf{d}\left(\mathbf{p}, \mathbf{p}^{\prime}\right)\right] \\
& \dot{b}(\mathbf{p}, t)=-\frac{i}{\hbar}\left[\left(\frac{\hbar^{2} p^{2}}{2 m}+E_{0}-E_{10}\right) b(\mathbf{p}, t)+\mathbf{E}(t) \cdot a(t) \mathbf{d}^{*}(\mathbf{p})+\mathbf{E}(t) \cdot \int d^{3} \mathbf{p}^{\prime} b\left(\mathbf{p}^{\prime}, t\right) \mathbf{g}\left(\mathbf{p}, \mathbf{p}^{\prime}\right)\right. \\
&\left.+\mathbf{E}(t) \cdot \sum_{\eta \neq 0} \int d^{3} \mathbf{p}^{\prime} c\left(\mathbf{p}^{\prime}, \eta, t\right) \mathbf{g}\left(\mathbf{p}, \mathbf{p}^{\prime}, \eta\right)+\mathbf{E}(t) \cdot \iint d^{3} \mathbf{p}^{\prime} d^{3} \mathbf{p}^{\prime \prime} d\left(\mathbf{p}^{\prime}, \mathbf{p}^{\prime \prime}, t\right) \mathbf{g}\left(\mathbf{p}, \mathbf{p}^{\prime}, \mathbf{p}^{\prime \prime}\right)\right] \\
& \dot{c}(\mathbf{p}, \eta, t)=-\frac{i}{\hbar}\left[\left(\frac{\hbar^{2} p^{2}}{2 m}+E_{0}-E_{1 \eta}\right) c(\mathbf{p}, \eta, t)+\mathbf{E}(t) \cdot a(t) \mathbf{d}^{*}(\mathbf{p}, \eta)+\mathbf{E}(t) \cdot \int d^{3} \mathbf{p}^{\prime} b\left(\mathbf{p}^{\prime}, t\right) \mathbf{g}^{*}\left(\mathbf{p}^{\prime}, \mathbf{p}, \eta\right)\right.
\end{aligned}
$$




$$
\begin{aligned}
+ & \left.\mathbf{E}(t) \cdot \sum_{\eta^{\prime} \neq 0} \int d^{3} \mathbf{p}^{\prime} c\left(\mathbf{p}^{\prime}, \eta^{\prime}, t\right) \mathbf{h}\left(\mathbf{p}, \eta, \mathbf{p}^{\prime}, \eta^{\prime}\right)+\mathbf{E}(t) \cdot \iint d^{3} \mathbf{p}^{\prime} d^{3} \mathbf{p}^{\prime \prime} d\left(\mathbf{p}^{\prime}, \mathbf{p}^{\prime \prime}, t\right) \mathbf{h}\left(\mathbf{p}, \eta, \mathbf{p}^{\prime}, \mathbf{p}^{\prime \prime}\right)\right] \\
\dot{d}\left(\mathbf{p}, \mathbf{p}^{\prime}, t\right)=-\frac{i}{\hbar}[ & \left(\frac{\hbar^{2} p^{2}}{2 m}+\frac{\hbar^{2} p^{\prime 2}}{2 m}+E_{0}\right) d\left(\mathbf{p}, \mathbf{p}^{\prime}, t\right)+\mathbf{E}(t) \cdot a(t) \mathbf{d}^{*}\left(\mathbf{p}^{\prime}, \mathbf{p}\right)+\mathbf{E}(t) \cdot \int d^{3} \mathbf{p}^{\prime \prime} b\left(\mathbf{p}^{\prime \prime}, t\right) \mathbf{g}^{*}\left(\mathbf{p}^{\prime \prime}, \mathbf{p}, \mathbf{p}^{\prime}\right) \\
+ & \left.\mathbf{E}(t) \cdot \sum_{\eta \neq 0} \int d^{3} \mathbf{p}^{\prime \prime} c\left(\mathbf{p}^{\prime \prime}, \eta, t\right) \mathbf{h}^{*}\left(\mathbf{p}^{\prime \prime}, \eta, \mathbf{p}, \mathbf{p}^{\prime}\right)+\mathbf{E}(t) \cdot \iint d^{3} \mathbf{p}^{\prime \prime} d^{3} \mathbf{p}^{\prime \prime \prime} d\left(\mathbf{p}^{\prime \prime}, \mathbf{p}^{\prime \prime \prime}, t\right) \mathbf{i}\left(\mathbf{p}, \mathbf{p}^{\prime}, \mathbf{p}^{\prime \prime}, \mathbf{p}^{\prime \prime \prime}\right)\right]
\end{aligned}
$$

\section{Appendix F: RESI and EII using perturbative expressions for matrix elements}

RESI We have left the dipole matrix element $\mathbf{g}\left(\mathbf{p}, \mathbf{p}^{\prime}, \eta\right)$ unevaluated as we will deal with this next. We need to take into account interactions here and to do this we can use first order perturbation theory. In the one-electron case, to include a direct and 'rescattering'-like contribution a perturbative series was used on the coefficients themselves. In this case in order to get a more explicit form of the electron-electron contribution we will use perturbation theory on the states themselves. We can split the states into a non-interacting part and a first order approximation to the interacting part as shown here

$$
|\mathbf{p}, 0\rangle=\left|\psi_{0}(\mathbf{p})\right\rangle+\left|\psi_{1}(\mathbf{p})\right\rangle,
$$

where $\left|\psi_{0}(\mathbf{p})\right\rangle$ is given by the non-interacting states for $|\mathbf{p}, 0\rangle$ and

$$
\left|\psi_{1}(\mathbf{p})\right\rangle=\sum_{\eta^{\prime} \neq 0} \int d^{3} \mathbf{p}^{\prime \prime} \frac{2 m\left\langle\psi_{0}\left(\mathbf{p}^{\prime}, \eta\right)\left|V_{12}\right| \psi_{0}(\mathbf{p})\right\rangle}{\hbar^{2}\left(p^{2}-p^{2}\right)-2 m\left(E_{10}-E_{1 \eta}\right)}\left|\psi_{0}\left(\mathbf{p}^{\prime \prime}, \eta^{\prime}\right)\right\rangle,
$$

where $\left|\psi_{0}\left(\mathbf{p}^{\prime}, \eta\right)\right\rangle$ is given by the non-interacting states for $|\mathbf{p}, \eta\rangle$. In the basis used in the perturbative expansion we have used excited states but also including the grounds state, i.e. $\eta=0$ as a basis to expand in. This is key to including transition from the ground state to excited states via electron interaction. Substituting this into the matrix element gives,

$$
\begin{aligned}
\mathbf{g}\left(\mathbf{p}, \mathbf{p}^{\prime}, \eta\right) & =\left\langle\mathbf{p}, 0\left|e\left(\hat{\mathbf{r}}_{1}+\hat{\mathbf{r}}_{2}\right)\right| \mathbf{p}^{\prime}, \eta\right\rangle \\
& =\left\langle\psi_{0}(\mathbf{p})\left|e\left(\hat{\mathbf{r}}_{1}+\hat{\mathbf{r}}_{2}\right)\right| \mathbf{p}^{\prime}, \eta\right\rangle+\left\langle\psi_{1}(\mathbf{p})\left|e\left(\hat{\mathbf{r}}_{1}+\hat{\mathbf{r}}_{2}\right)\right| \mathbf{p}^{\prime}, \eta\right\rangle
\end{aligned}
$$

The first part is just given by the non-interacting form that we previously calculated for this matrix element, while the for the second we can insert the Eq. (F2)

$$
\begin{aligned}
& \mathbf{g}\left(\mathbf{p}, \mathbf{p}^{\prime}, \eta\right)= e \delta\left(\mathbf{p}-\mathbf{p}^{\prime}\right)\langle 0|\hat{\mathbf{r}}| \eta\rangle+ \\
& \sum_{\eta^{\prime} \neq 0} \int d^{3} \mathbf{p}^{\prime \prime}\left(\frac{2 m e\left\langle\psi_{0}\left(\mathbf{p}^{\prime}, \eta\right)\left|V_{12}\right| \psi_{0}(\mathbf{p})\right\rangle}{\hbar^{2}\left(p^{2}-p^{\prime 2}\right)-2 m\left(E_{10}-E_{1 \eta}\right)}\right)^{*} \underbrace{\left\langle\psi_{0}\left(\mathbf{p}^{\prime \prime}, \eta^{\prime}\right)\left|e\left(\hat{\mathbf{r}}_{1}+\hat{\mathbf{r}}_{2}\right)\right| \mathbf{p}^{\prime}, \eta\right\rangle}_{\eta^{\prime} \neq 0} \\
&=e \delta\left(\mathbf{p}-\mathbf{p}^{\prime \prime}, \eta^{\prime}, \mathbf{p}^{\prime}, \eta\right) \\
& \quad \times\left(\frac{2 m e\left\langle\psi_{0}(\mathbf{p})\left|V_{12}\right| \psi_{0}\left(\mathbf{p}^{\prime}, \eta\right)\right\rangle}{\hbar^{2}\left(p^{2}-p^{\prime 2}\right)-2 m\left(E_{10}-E_{1 \eta}\right)}\right) \\
&=e \delta\left(\mathbf{p}-\mathbf{p}^{\prime}\right)\langle 0|\hat{\mathbf{r}}| \eta\rangle+\sum^{3} \mathbf{p}^{\prime \prime}\left(\delta_{\eta^{\prime} \eta}\left(i e \hbar \nabla_{\mathbf{p}^{\prime \prime}} \delta\left(\mathbf{p}^{\prime \prime}-\mathbf{p}^{\prime}\right)+\hbar \tilde{\mathbf{g}}\left(\mathbf{p}^{\prime \prime}, \mathbf{p}^{\prime}\right)\right)+e \delta\left(\mathbf{p}^{\prime \prime}-\mathbf{p}^{\prime}\right)\left\langle\eta^{\prime}|\hat{\mathbf{r}}| \eta\right\rangle\right) \\
&+\nabla_{\mathbf{p}^{\prime}} \frac{2 m e\left\langle\psi_{0}(\mathbf{p})\left|V_{12}\right| \psi_{0}\left(\mathbf{p}^{\prime}, \eta\right)\right\rangle}{\hbar^{2}\left(p^{2}-p^{\prime 2}\right)-2 m\left(E_{10}-E_{1 \eta}\right)} \\
&+\int d^{3} \mathbf{p}^{\prime \prime} \frac{2 m e\left\langle\psi_{0}(\mathbf{p})\left|V_{12}\right| \psi_{0}\left(\mathbf{p}^{\prime}, \eta\right)\right\rangle}{\hbar^{2}\left(p^{2}-p^{\prime 2}\right)-2 m\left(E_{10}-E_{1 \eta}\right)} \tilde{g}\left(\mathbf{p}^{\prime \prime}, \mathbf{p}^{\prime}\right) \\
&+\sum_{\eta^{\prime} \neq 0} \frac{e}{\Delta E}\left\langle\psi_{0}(\mathbf{p})\left|V_{12}\right| \psi_{0}\left(\mathbf{p}^{\prime}, \eta^{\prime}\right)\right\rangle\left\langle\eta^{\prime}|\hat{\mathbf{r}}| \eta\right\rangle
\end{aligned}
$$

The last line shows the different pathways we have revealed by this expansion. Each term in the above equation relates to a different physical process, the first is where the ground state is excited to by the laser while the scattering 
state is unaffected. The second term relates to the continuum electron being driven by the laser to the interact with ground state electron causing it to be excited, this is the most relevant one for RESI. These first two are shown in Fig. 13. The third term is similar to the second except there is an additional interaction with the core for the continuum electron, this could be from re-scattering after the RESI process has taken place. The final term excites the second electron in two steps through electron interaction and the laser via an intermediate state. An additional pathway can be revealed if the same expansion is applied to the right ket state of Eq. (F3). This pathway is the same as the latter except the order of the interactions is reversed and laser excites the electron into the intermediate state from the ground state. We did not consider this, given these pathways will not contribute to the core of the RESI process.

Now we make the assumption that there will be no laser induced transitions between bound states and the matrix element simplifies it to

$$
\mathbf{g}\left(\mathbf{p}, \mathbf{p}^{\prime}, \eta\right)=\nabla_{\mathbf{p}^{\prime}} \frac{2 m e\left\langle\psi_{0}(\mathbf{p})\left|V_{12}\right| \psi_{0}\left(\mathbf{p}^{\prime}, \eta\right)\right\rangle}{\hbar^{2}\left(p^{2}-p^{\prime 2}\right)-2 m\left(E_{10}-E_{1 \eta}\right)} .
$$

This assumption also simplifies the integro-differential equations and we can insert the calculation we performed for $\mathbf{g}\left(\mathbf{p}, \mathbf{p}^{\prime}, \eta\right)$, this yields

$$
\begin{aligned}
& \dot{a}(t)=-\frac{2 i e}{\hbar} \mathbf{E}(t) \cdot \int d^{3} \mathbf{p}\left\langle 0_{1}|\hat{\mathbf{r}}| \mathbf{p}\right\rangle b(\mathbf{p}, t) \\
& \dot{b}(\mathbf{p}, t)=-\frac{i}{\hbar}\left[\left(\frac{\hbar^{2} p^{2}}{2 m}+E_{0}-E_{10}\right) b(\mathbf{p}, t)+\frac{2 e \mathbf{E}(t)}{\sqrt{2}} a(t)\left\langle\mathbf{p}|\hat{\mathbf{r}}| 0_{1}\right\rangle+i e \hbar \mathbf{E}(t) \cdot \nabla_{\mathbf{p}} b(\mathbf{p}, t)\right. \\
&\left.+\mathbf{E}(t) \cdot \sum_{\eta \neq 0} \int d^{3} \mathbf{p}^{\prime} \nabla_{\mathbf{p}^{\prime}} \frac{2 m e\left\langle\psi_{0}(\mathbf{p})\left|V_{12}\right| \psi_{0}\left(\mathbf{p}^{\prime}, \eta\right)\right\rangle}{\hbar^{2}\left(p^{2}-p^{\prime 2}\right)-2 m\left(E_{10}-E_{1 \eta}\right)} c(\mathbf{p}, \eta, t)\right] \\
& \dot{c}(\mathbf{p}, \eta, t)=-\frac{i}{\hbar}\left[\left(\frac{\hbar^{2} p^{2}}{2 m}+E_{0}-E_{1 \eta}\right) c(\mathbf{p}, \eta, t)+\mathbf{E}(t) \cdot \int d^{3} \mathbf{p}^{\prime} \nabla_{\mathbf{p}} \frac{2 m e\left\langle\psi_{0}(\mathbf{p}, \eta)\left|V_{12}\right| \psi_{0}\left(\mathbf{p}^{\prime}\right)\right\rangle}{\hbar^{2}\left(p^{\prime 2}-p^{2}\right)-2 m\left(E_{10}-E_{1 \eta}\right)} b\left(\mathbf{p}^{\prime}, t\right)\right. \\
&\left.+i e \hbar \mathbf{E}(t) \nabla_{\mathbf{p}} c(\mathbf{p}, \eta, t)+2 e \mathbf{E}(t) \cdot \int d^{3} \mathbf{p}^{\prime}\left\langle\eta|\hat{\mathbf{r}}| \mathbf{p}^{\prime}\right\rangle d\left(\mathbf{p}, \mathbf{p}^{\prime}, t\right)\right] \\
& \dot{d}\left(\mathbf{p}, \mathbf{p}^{\prime}, t\right)=-\frac{i}{\hbar}\left[\left(\frac{\hbar^{2} p^{2}}{2 m}+\frac{\hbar^{2} p^{\prime 2}}{2 m}+E_{0}\right) d\left(\mathbf{p}, \mathbf{p}^{\prime}, t\right)+e \mathbf{E}(t) \cdot \sum_{\eta \neq 0}\left(c\left(\mathbf{p}^{\prime}, \eta, t\right)\langle\mathbf{p}|\hat{\mathbf{r}}| \eta\rangle+c(\mathbf{p}, \eta, t)\left\langle\mathbf{p}^{\prime}|\hat{\mathbf{r}}| \eta\right\rangle\right)\right. \\
&\left.+\mathbf{E}(t) \cdot\left(\nabla_{\mathbf{p}}+\nabla_{\mathbf{p}^{\prime}}\right)\left(d\left(\mathbf{p}, \mathbf{p}^{\prime}, t\right)+d\left(\mathbf{p}^{\prime}, \mathbf{p}, t\right)\right)\right]
\end{aligned}
$$

Now we select only terms that contribute to the final probability flow into double ionized states and ignore the flow in the opposite direction, as such we will assume $a(t)=1$ is unity throughout. This simplifies the integro-differential equations into a solvable form,

$$
\begin{aligned}
\dot{b}(\mathbf{p}, t)=-\frac{i}{\hbar} & {\left[\left(\frac{\hbar^{2} p^{2}}{2 m}+E_{0}-E_{10}\right) b(\mathbf{p}, t)+\frac{2 e \mathbf{E}(t)}{\sqrt{2}}\left\langle\mathbf{p}|\hat{\mathbf{r}}| 0_{1}\right\rangle+i e \hbar \mathbf{E}(t) \cdot \nabla_{\mathbf{p}} b(\mathbf{p}, t)\right] } \\
\dot{c}(\mathbf{p}, \eta, t)=-\frac{i}{\hbar}[ & \left(\frac{\hbar^{2} p^{2}}{2 m}+E_{0}-E_{1 \eta}\right) c(\mathbf{p}, \eta, t)+\mathbf{E}(t) \cdot \int d^{3} \mathbf{p}^{\prime} \nabla_{\mathbf{p}} \frac{2 m e\left\langle\psi_{0}(\mathbf{p}, \eta)\left|V_{12}\right| \psi_{0}\left(\mathbf{p}^{\prime}\right)\right\rangle}{\hbar^{2}\left(p^{\prime 2}-p^{2}\right)-2 m\left(E_{10}-E_{1 \eta}\right)} b\left(\mathbf{p}^{\prime}, t\right) \\
& \left.+i e \hbar \mathbf{E}(t) \nabla_{\mathbf{p}} c(\mathbf{p}, \eta, t)\right] \\
\dot{d}\left(\mathbf{p}, \mathbf{p}^{\prime}, t\right)=-\frac{i}{\hbar} & {\left[\left(\frac{\hbar^{2} p^{2}}{2 m}+\frac{\hbar^{2} p^{\prime 2}}{2 m}+E_{0}\right) d\left(\mathbf{p}, \mathbf{p}^{\prime}, t\right)+e \mathbf{E}(t) \cdot \sum_{\eta \neq 0}\left(c\left(\mathbf{p}^{\prime}, \eta, t\right)\langle\mathbf{p}|\hat{\mathbf{r}}| \eta\rangle+c(\mathbf{p}, \eta, t)\left\langle\mathbf{p}^{\prime}|\hat{\mathbf{r}}| \eta\right\rangle\right)\right.} \\
& \left.+\mathbf{E}(t) \cdot\left(\nabla_{\mathbf{p}}+\nabla_{\mathbf{p}^{\prime}}\right)\left(d\left(\mathbf{p}, \mathbf{p}^{\prime}, t\right)+d\left(\mathbf{p}^{\prime}, \mathbf{p}, t\right)\right)\right]
\end{aligned}
$$


EII Here, we neglect the dipole matrix element for recollision excitation RESI contribution given by $\mathbf{g}\left(\mathbf{p}, \mathbf{p}^{\prime}, \eta\right)$, and instead include in a similar way the matrix element $\mathbf{g}\left(\mathbf{p}, \mathbf{p}^{\prime}, \mathbf{p}^{\prime \prime}\right)$. We can proceed as before splitting the wavefunction into an non-interacting part and an interacting perturbation, the perturbation is given by

$$
\left|\psi_{1}(\mathbf{p})\right\rangle=\iint d^{3} \mathbf{k} d^{3} \mathbf{k}^{\prime} \frac{2 m\left\langle\psi_{0}\left(\mathbf{k}, \mathbf{k}^{\prime}\right)\left|V_{12}\right| \psi_{0}(\mathbf{p})\right\rangle}{\hbar^{2}\left(\mathbf{p}-\mathbf{k}-\mathbf{k}^{\prime}\right)-E_{10}}\left|\psi_{0}\left(\mathbf{k}, \mathbf{k}^{\prime}\right)\right\rangle .
$$

Then removing dipole transitions for the second electron from the bound state to the continuum to keep only the EI mechanism for the $\mathbf{g}\left(\mathbf{p}, \mathbf{p}^{\prime}, \mathbf{p}^{\prime \prime}\right)$ matrix elements yields

$$
\mathbf{g}\left(\mathbf{p}, \mathbf{p}^{\prime}, \mathbf{p}^{\prime \prime}\right)=2\left(\nabla_{\mathbf{p}^{\prime}}+\nabla_{\mathbf{p}^{\prime \prime}}\right) \frac{\left.2 m\left\langle\psi_{0}\left(\mathbf{p}^{\prime}, \mathbf{p}^{\prime \prime}\right)\left|V_{12}\right| \psi_{0}(\mathbf{p})\right)\right\rangle}{\hbar^{2}\left(p^{2}-p^{\prime 2}-p^{\prime \prime 2}-E_{10}\right)}
$$

Now the integro-differential equation for $d\left(\mathbf{p}, \mathbf{p}^{\prime}, t\right)$ can be written out, this time it only depends on $b\left(\mathbf{p}^{\prime \prime}, t^{\prime \prime}\right)$ and is given by,

$$
\begin{gathered}
\dot{d}\left(\mathbf{p}, \mathbf{p}^{\prime}, t\right)=-\frac{i}{\hbar}\left[\left(\frac{\hbar^{2} p^{2}}{2 m}+\frac{\hbar^{2} p^{\prime 2}}{2 m}+E_{0}\right) d\left(\mathbf{p}, \mathbf{p}^{\prime}, t\right)+2 \mathbf{E}(t) \cdot \int d^{3} \mathbf{p}^{\prime \prime} b\left(\mathbf{p}^{\prime \prime}, t^{\prime \prime}\right)\left(\nabla_{\mathbf{p}}+\nabla_{\mathbf{p}^{\prime}}\right) \frac{2 m\left\langle\psi_{0}\left(\mathbf{p}^{\prime \prime}\right)\left|V_{12}\right| \psi_{0}\left(\mathbf{p}, \mathbf{p}^{\prime}\right)\right\rangle}{\hbar\left(p^{\prime 2}-p^{2}-p^{\prime 2}\right)-E_{10}}\right. \\
\left.+\mathbf{E}(t) \cdot\left(\nabla_{\mathbf{p}}+\nabla_{\mathbf{p}^{\prime}}\right)\left(d\left(\mathbf{p}, \mathbf{p}^{\prime}, t\right)+d\left(\mathbf{p}^{\prime}, \mathbf{p}, t\right)\right)\right]
\end{gathered}
$$

Then the solution can be written as

$$
\begin{aligned}
& d\left(\mathbf{p}, \mathbf{p}^{\prime}, t\right)=i \int_{0}^{t} d t^{\prime \prime} 2 e \mathbf{E}\left(t^{\prime \prime}\right) \cdot \int d^{3} \mathbf{p}^{\prime \prime} b\left(\mathbf{p}^{\prime \prime}, t^{\prime \prime}\right)\left(\nabla_{\mathbf{p}}+\nabla_{\mathbf{p}^{\prime}}\right) \frac{2 m\left\langle\psi_{0}\left(\mathbf{p}^{\prime \prime}\right)\left|V_{12}\right| \psi_{0}\left(\mathbf{p}-\frac{e}{c} \mathbf{A}\left(t^{\prime \prime}\right), \mathbf{p}^{\prime}-\frac{e}{c} \mathbf{A}\left(t^{\prime \prime}\right)\right)\right\rangle}{\hbar\left(p^{\prime \prime 2}-\left(p-\frac{e}{c} \mathbf{A}\left(t^{\prime \prime}\right)\right)^{2}-\left(p^{\prime}-\frac{e}{c} \mathbf{A}\left(t^{\prime \prime}\right)\right)^{2}\right)-E_{10}} \\
& \times \exp \left[\frac{i}{\hbar} S_{d}\left(\mathbf{p}, \mathbf{p}^{\prime}, t^{\prime \prime}, t\right)\right]
\end{aligned}
$$

Using the same equations for $b\left(\mathbf{p}^{\prime \prime}, t^{\prime \prime}\right)$ and $S_{d}\left(\mathbf{p}, \mathbf{p}^{\prime}, t^{\prime \prime}, t\right)$ can be used as before. Additionally both the EI and RESI mechanism can be included in $d\left(\mathbf{p}, \mathbf{p}^{\prime}, t\right)$ and it will still be integrable and, as expected, will simply be equal to the sum of these two solutions. 